\title{
EFFECTIVE CARDINALS OF BOLDFACE POINTCLASSES
}

\author{
ALESSANDRO ANDRETTA, GREG HJORTH, AND ITAY NEEMAN
}

\begin{abstract}
Assuming AD $+D C(\mathbb{R})$, we characterize the self-dual boldface pointclasses which are strictly larger (in terms of cardinality) than the pointclasses contained in them: these are exactly the clopen sets, the collections of all sets of Wadge rank $\leq \omega_{1}^{\xi}$, and those of Wadge rank $<\omega_{1}^{\xi}$ when $\xi$ is limit.
\end{abstract}

\section{INTRODUCTION}

A boldface pointclass (for short: a pointclass) is a non-empty collection $\Gamma$ of subsets of $\mathbb{R}$ such that $\Gamma$ is closed under continuous pre-images and $\Gamma \neq \mathscr{P}(\mathbb{R})$. Examples of pointclasses are the levels $\Sigma_{\alpha}^{0}, \boldsymbol{\Pi}_{\alpha}^{0}, \boldsymbol{\Delta}_{\alpha}^{0}$ of the Borel hierarchy and the levels $\Sigma_{n}^{1}, \Pi_{n}^{1}, \Delta_{n}^{1}$ of the projective hierarchy. In this paper we address the following

Question 1. What is the cardinality of a pointclass?

Assuming AC, the Axiom of Choice, Question 1 becomes trivial for all pointclasses $\Gamma$ which admit a complete set. These pointclasses all have size $2^{\aleph_{0}}$ under AC. On the other hand there is no obvious, natural way to associate, in a one-to-one way, an open set (or for that matter: a closed set, or a real number) to any $\boldsymbol{\Sigma}_{2}^{0}$ set. This suggests that in the realm of definable sets and functions already $\Sigma_{1}^{0}$ and $\Sigma_{2}^{0}$ may have different sizes. Indeed the second author in [Hjo98] and [Hjo02] showed that $\mathrm{AD}+\mathrm{V}=\mathrm{L}(\mathbb{R})$ actually implies

(a) $1 \leq \alpha<\beta<\omega_{1} \Longrightarrow\left|\Sigma_{\alpha}^{0}\right|<\left|\Sigma_{\beta}^{0}\right|$, and

(b) $\left|\Delta_{1}^{1}\right|<\left|\Sigma_{1}^{1}\right|<\left|\Sigma_{2}^{1}\right|<\ldots$

(Since we do not assume Choice, cardinal inequalities are to be understood as follows: for any sets $X$ and $Y,|X| \leq|Y|$ means that there is an injection of $X$ into $Y$, $|X|<|Y|$ means that $|X| \leq|Y| \&|Y| \not|X| X \mid$, and $|X|=|Y|$ means that $|X| \leq$ $|Y| \leq|X|$ or, equivalently (by the Shroeder-Bernstein Theorem), that there is a bijection between $X$ and $Y$.) The third author strengthened the result in (a) by showing that $\left|\boldsymbol{\Delta}_{\alpha+1}^{0}\right|<\left|\Sigma_{\alpha+1}^{0}\right|$, all $1 \leq \alpha<\omega_{1}$. Therefore, in the AD-world, the answer to Question 1 is far from being trivial.

The results mentioned above did not characterize completely the cardinality pointclasses, that is those $\boldsymbol{\Gamma}$ such that $\left|\Gamma^{\prime}\right|<|\boldsymbol{\Gamma}|$, for any $\boldsymbol{\Gamma}^{\prime} \subset \boldsymbol{\Gamma}$. For example they said nothing about the existence of cardinality pointclasses strictly between $\boldsymbol{\Sigma}_{2}^{0} \cup \boldsymbol{\Pi}_{2}^{0}$ and $\Delta_{3}^{0}$. The main result of this paper is a complete characterization, under AD, of all cardinality pointclasses in terms of their Wadge rank. (The notion of Wadge rank

1991 Mathematics Subject Classification. 03E15.

Key words and phrases. Determinacy, Wadge hierarchy. 
and other relevant facts from Descriptive Set Theory are recalled in sections 2 and 3.) In order to state our characterization of cardinality pointclasses, we need an auxiliary definition. Since $|\boldsymbol{\Gamma}|=|\boldsymbol{\Gamma} \cup \breve{\boldsymbol{\Gamma}}|$ for any pointclass $\boldsymbol{\Gamma}$ containing $\boldsymbol{\Delta}_{1}^{0}$ (see Corollary 19 below), it is enough to restrict our attention to self-dual pointclasses. By AD the self-dual pointclasses are exactly the levels of the Wadge hierarchy - that is, they are of the form

$$
\mathscr{P}^{(\alpha)}(\mathbb{R})=\text { the collection of all sets of Wadge rank }<\alpha .
$$

Say that a self-dual $\boldsymbol{\Delta}$ is a cardinality level just in case $\left|\boldsymbol{\Delta}^{\prime}\right|<|\boldsymbol{\Delta}|$ for any self-dual $\boldsymbol{\Delta}^{\prime} \subset \boldsymbol{\Delta}$. Since cardinality pointclasses correspond to cardinality levels, determining all cardinality pointclasses amounts to pinning-down those ordinals $\alpha$ for which $\mathscr{P}^{(\alpha)}(\mathbb{R})$ is a cardinality level. In this paper a description of such ordinals is given, providing thus a complete answer to Question 1.

Theorem 1. Assume $\mathrm{AD}+\mathrm{DC}(\mathbb{R})$. Then $\mathscr{P}^{(\alpha)}(\mathbb{R})$ is a cardinality level iff $\alpha=3$ or

$$
\exists \xi<\Theta\left(\alpha=\omega_{1}^{\xi}+1 \vee\left(\alpha=\omega_{1}^{\xi} \& \xi \text { is limit }\right)\right) .
$$

$\operatorname{DC}(\mathbb{R})$ is the Axiom of Dependent Choices over the reals, and $\Theta$ is the supremum of the ordinals which are the surjective image of $\mathbb{R}$,

$$
\Theta=\sup \{\alpha \mid \exists f: \mathbb{R} \rightarrow \alpha\},
$$

and it is also the length of the Wadge hierarchy.

Theorem 1 subsumes all previous results - see Corollary 27. For example, by [Wad83] the Wadge rank of a complete $\Sigma_{\alpha}^{0}$ set is $\omega_{1}^{\xi}$, with $\xi$ limit of uncountable cofinality if $\alpha \geq 3$, and $\xi=1$ when $\alpha=2$. Notice that

$$
\mathscr{P}^{\left(\omega_{1}^{\xi}+1\right)}(\mathbb{R})=\Sigma_{\alpha}^{0} \cup \boldsymbol{\Pi}_{\alpha}^{0} \quad \text { and } \quad \mathscr{P}^{\left(\omega_{1}^{\xi}\right)}(\mathbb{R})=\boldsymbol{\Delta}_{\alpha}^{0}
$$

for this $\xi$. Using Theorem 1 it follows that for $2 \leq \beta<\alpha<\omega_{1}$,

$$
\left|\Sigma_{\beta}^{0}\right|<\left|\Delta_{\alpha}^{0}\right|<\left|\Sigma_{\alpha}^{0}\right|=\left|\Sigma_{\alpha}^{0} \cup \Pi_{\alpha}^{0}\right| \text {. }
$$

(The case when $\beta=1$ and $\alpha=2$ is a bit different as $\left|\boldsymbol{\Sigma}_{1}^{0}\right|=\left|\boldsymbol{\Delta}_{2}^{0}\right|=|\mathbb{R}|$.)

Assuming AD, each pointclass $\boldsymbol{\Gamma}$ is the surjective image of $\mathbb{R}$, so it is in bijection with $\mathbb{R} / E$ for some equivalence relation $E$ on $\mathbb{R}$. Conversely, suppose $E$ is an equivalence relation on $\mathbb{R}$, and let $\alpha$ be the Wadge rank of $E$ (after identifying $\mathbb{R} \times \mathbb{R}$ with $\mathbb{R})$. Then every equivalence class $[x]_{E}$ is the continuous pre-image of $E \cap(\{x\} \times \mathbb{R})$, which is in $\mathscr{P}^{(\alpha+1)}(\mathbb{R})$ (if $\alpha \geq \omega$-see Lemma 6 below), since it is the intersection of a set of Wadge rank $\alpha$ and a closed set, and hence $\mathbb{R} / E \subseteq \mathscr{P}(\alpha+1)(\mathbb{R})$. Therefore the cardinalities of the pointclasses $\mathscr{P}^{(\alpha)}(\mathbb{R})$ as in Theorem 1 are cofinal in the cardinalities of quotients of $\mathbb{R}$.

Theorem 1 is stated and proved under AD, but since determinacy is used in a "local" way, weaker versions of the theorem - compatible with AC - can be extracted from its proof. For example, suppose we restrict ourselves to Borel pointclasses, that is pointclasses contained in $\boldsymbol{\Delta}_{1}^{1}$. Thus a self-dual Borel pointclass is of the form $\mathscr{P}^{(\alpha)}(\mathbb{R})$ with $\alpha \leq \eta$, where $\eta$ is the Wadge rank of a complete $\boldsymbol{\Sigma}_{1}^{1}$ set or, 
equivalently, of a complete $\boldsymbol{\Pi}_{1}^{1}$ set. Each Borel pointclass is the surjective image of $\mathbb{R}$, i.e., there is a $\pi_{\Gamma}: \mathbb{R} \rightarrow \Gamma$ and moreover such $\pi_{\Gamma}$ can be taken so that the equivalence relation

$$
x \sim_{\Gamma} y \Longleftrightarrow \pi_{\Gamma}(x)=\pi_{\Gamma}(y)
$$

is $\boldsymbol{\Pi}_{1}^{1}$. A function $F: \Gamma \rightarrow \Lambda$ between Borel pointclasses is said to be Borel-in-thecodes if there is a Borel $\hat{F}: \mathbb{R} \rightarrow \mathbb{R}$ such that

$$
x \sim_{\boldsymbol{\Gamma}} y \Longrightarrow \hat{F}(x) \sim_{\boldsymbol{\Lambda}} \hat{F}(y)
$$

and $F(A)=\pi_{\boldsymbol{\Lambda}}(\hat{F}(x))$, for some/any $x \in \mathbb{R}$ such that $\pi_{\boldsymbol{\Gamma}}(x)=A$. Let us say that a self-dual Borel pointclass $\mathscr{P}^{(\alpha)}(\mathbb{R})$ is a $\boldsymbol{\Delta}_{1}^{1}$-cardinality level if it does not admit a Borel-in-the-codes injection into some $\mathscr{P}^{(\beta)}(\mathbb{R})$, with $\beta<\alpha$. Then the proof of Theorem 1 yields:

Theorem $\mathbf{1}^{\prime}(\mathrm{ZFC})$. If $\mathscr{P}^{(\alpha)}(\mathbb{R}) \subseteq \boldsymbol{\Delta}_{1}^{1}$, then $\mathscr{P}^{(\alpha)}(\mathbb{R})$ is a $\boldsymbol{\Delta}_{1}^{1}$-cardinality level iff $\alpha=3$ or

$$
\exists \xi\left(\alpha=\omega_{1}^{\xi}+1 \vee\left(\alpha=\omega_{1}^{\xi} \& \xi \text { is limit }\right)\right) .
$$

Similarly, if we assume Projective Determinacy and restrict the injections between projective pointclasses to be projective-in-the-codes, we have a characterization of all projective pointclasses which are projective-cardinality level.

The paper is organized as follows: after presenting the notations and conventions in $\S 2$, we summarize the relevant results on the Wadge hierarchy in $\S 3$. The basic facts about cardinalities of pointclasses are proved in $\S 4$, while the proof of the key result (Theorem 29) for showing Theorem 1 is in $\S 5$. In the Appendix a proof of a special case of Theorem 1 is sketched, stating that there are more $2-\boldsymbol{\Sigma}_{2}^{0}$ sets (i.e., sets of the form $F \backslash G$ with $F, G \in \Sigma_{2}^{0}$ ) than $\Sigma_{2}^{0}$ sets. This was our first result and its proof-properly generalized-yielded the main result of this paper. We hope that this proof (which can be read independently from the rest of the paper) will be helpful to get a better understanding of the arguments in Sections 4 and 5 .

\section{Preliminaries}

As customary in set theory, $\mathbb{R}$ denotes the Baire space ${ }^{\omega} \omega$, with the topology generated by the metric

$$
d(x, y)= \begin{cases}0 & \text { if } x=y, \\ 2^{-n} & \text { if } n \text { is least such that } x(n) \neq y(n) .\end{cases}
$$

Thus the basic open sets are of the form $\boldsymbol{N}_{s}=\{x \in \mathbb{R} \mid x \supset s\}$, with $s \in{ }^{<\omega} \omega$.

For $A \subseteq \mathbb{R}$, the interior of $A$ is denoted by $\operatorname{Int}(A)$, and the complement $\mathbb{R} \backslash A$ of $A$ is denoted by $\neg A$.

A tree on $\omega$ is a $T \subseteq{ }^{<\omega} \omega$ which is closed under initial segments; it is pruned iff $\forall t \in T \exists s \in T(t \subset s)$. The body of $T$ is the set $[T]=\{x \in \mathbb{R} \mid \forall n(x\lceil n \in T)\}$ of all infinite branches of $T$; $[T]$ is a closed subset of $\mathbb{R}$, and all closed subsets of $\mathbb{R}$ are 
of this form with $T$ pruned. The boundary of $T$ is the set of all sequences that just left $T$, that is

$$
\partial T=\left\{s \in{ }^{<\omega} \omega \mid s \notin T \& s\lceil\operatorname{lh}(s)-1 \in T\} .\right.
$$

If $s, t \in{ }^{<\omega} \omega$, then $s^{\frown} t$ is the concatenation of $s$ and $t$; when $t=\langle n\rangle$ we will often write $s^{\frown} n$ instead of $s^{\frown}\langle n\rangle$. The definition of $s^{\frown} t$ is extended in the obvious way to the case when $t$ is an infinite sequence, i.e., when $t \in \mathbb{R}$. For $x \in<^{<\omega} \omega \cup \mathbb{R}$ let $x+1=\langle x(n)+1 \mid n<\operatorname{lh}(x)\rangle$ and $x \dot{-} 1=\langle x(n) \dot{-} 1 \mid n<\operatorname{lh}(x)\rangle$, where, for $k \in \omega$,

$$
k-1= \begin{cases}k-1 & \text { if } k \geq 1, \\ 0 & \text { otherwise. }\end{cases}
$$

For $k, n \in \omega$ let $k^{(n)}$ be the sequence of $n$-many consecutive $k$ 's, that is, $k^{(0)}=\emptyset$ and $k^{(n+1)}=k^{(n)}\langle\langle k\rangle$.

For $s \in{ }^{<\omega} \omega, T$ a tree on $\omega$, and $A \subseteq \mathbb{R}$, let

$$
\begin{aligned}
& s^{\wedge} A=\left\{s^{\wedge} x \mid x \in A\right\}, \\
& A_{\lfloor s\rfloor}=\left\{x \mid s^{\frown} x \in A\right\}, \\
& T_{\lfloor s\rfloor}=\left\{t \mid s^{\wedge} t \in T\right\},
\end{aligned}
$$

so that $s^{\wedge} \mathbb{R}=\boldsymbol{N}_{s},\left(s^{\wedge} A\right)_{\lfloor s\rfloor}=A$, and $[T]_{\lfloor s\rfloor}=\left[T_{\lfloor s\rfloor}\right]$.

Let $S, T$ be pruned trees on $\omega$. A map $\Phi: S \rightarrow T$ is

monotone: if $\Phi(\emptyset)=\emptyset$ and $s \subseteq s^{\prime} \Longrightarrow \Phi(s) \subseteq \Phi\left(s^{\prime}\right)$,

Lipschitz: if it is monotone and $\operatorname{lh}(\Phi(s))=\operatorname{lh}(s)$,

continuous: if it is monotone and $\lim _{n \rightarrow \infty} \operatorname{lh}(\Phi(x\lceil n))=\infty$ for each $x \in[S]$.

Clearly, if $\Phi$ is Lipschitz, then $\Phi$ is continuous, but while the definition of "Lipschitz" makes sense even for finite trees, the definition of "continuous" is of interest only when $[S] \neq \emptyset$. If $S$ is pruned and non-empty and $\Phi: S \rightarrow T$ is continuous, then we can define a continuous function

$$
f_{\Phi}:[S] \rightarrow[T], \quad x \mapsto \bigcup_{n} \Phi(x\lceil n),
$$

and if $\Phi$ is Lipschitz, then $f_{\Phi}$ is Lipschitz with constant $\leq 1$ with respect to the metric that $[S]$ and $[T]$ inherit from $\mathbb{R}$, that is

$$
\forall x, y \in[S] \forall n\left(x \uparrow n=y \uparrow n \Longrightarrow f_{\Phi}(x) \uparrow n=f_{\Phi}(y) \uparrow n\right) .
$$

Every function $f:[S] \rightarrow[T]$ which is continuous (Lipschitz with constant $\leq 1$ ) is of the form $f_{\Phi}$, where $\Phi: S \rightarrow T$ is continuous (resp. Lipschitz). Since a continuous $f$ : $\mathbb{R} \rightarrow \mathbb{R}$ is completely determined by a continuous $\Phi:{ }^{<\omega} \omega \rightarrow{ }^{<\omega} \omega$, and since each $\Phi$ is - essentially - a subset of $\omega$, every continuous function can be coded by a real, i.e., a map $x \mapsto f_{x}$ can be defined so that $\left\{f_{x} \mid x \in \mathbb{R}\right\}=\{f \in \mathbb{R} \mathbb{R} \mid f$ is continuous $\}$. We call such a map a parametrization or coding of the continuous functions. A diagonal argument shows that no such a parametrization yields a continuous evaluation map $\mathbb{R}^{2} \rightarrow \mathbb{R},(x, y) \mapsto f_{x}(y)$. In order to achieve continuity we must restrict ourselves to Lipschitz functions: let

$$
\left\langle\boldsymbol{s}_{n} \mid n \in \omega\right\rangle
$$


be a standard enumeration without repetitions of $<\omega \omega \backslash\{\emptyset\}$ such that $\boldsymbol{s}_{n} \subset \boldsymbol{s}_{m} \Longrightarrow$ $n<m$. For any $x \in \mathbb{R}$ let $\Phi_{x}:{ }^{<\omega} \omega \rightarrow{ }^{<\omega} \omega$ be defined by

$$
\Phi_{x}(s)=t \Longleftrightarrow \operatorname{lh}(s)=\operatorname{lh}(t) \& \forall n \forall i<\operatorname{lh}(s)\left(s_{n}=s \uparrow i+1 \Longrightarrow t(i)=x(n)\right) .
$$

This is well-defined since $s \uparrow i+1=\boldsymbol{s}_{n}$ for a unique $n$, and clearly $\Phi_{x}$ is Lipschitz. Moreover, for every Lipschitz $\Phi:{ }^{<\omega} \omega \rightarrow{ }^{<\omega} \omega$ there is a unique $x$ such that $\Phi=\Phi_{x}$. Letting $\boldsymbol{\ell}_{x}: \mathbb{R} \rightarrow \mathbb{R}$ be the Lipschitz functions induced by $\Phi_{x}$, it is not hard to see that the evaluation map $(x, y) \mapsto \boldsymbol{\ell}_{x}(y)$, is continuous. Therefore we have shown that:

(3) The correspondence $x \mapsto \boldsymbol{\ell}_{x}$ is a bijection between $\mathbb{R}$ and $\{f \in \mathbb{R} \mathbb{R} \mid f$ is Lipschitz $\}$, and the map $\mathbb{R}^{2} \rightarrow \mathbb{R},(x, y) \mapsto \boldsymbol{\ell}_{x}(y)$, is continuous.

If $T$ is a pruned tree, then we can define a canonical Lipschitz map $\varrho_{T}:<\omega_{\omega} \rightarrow T$ as follows. For any $t \in T$ and $n>\operatorname{lh}(t)$, the left-most extension of $t$ in $T$ of length $n$ is the unique $s \in T$ such that $s \supset t, \operatorname{lh}(s)=n$ and it is lexicographically least among such $s$. Also, for $t \in{ }^{<\omega} \omega$ let $\hat{t}$ be the longest initial segment of $t$ inside $T$, i.e., $\hat{t}=t$ if $t \in T$ or $\hat{t}=t \uparrow n$ if $n+1 \in \operatorname{dom}(t)$ and $t \uparrow n+1 \in \partial T$. Let

$$
\varrho_{T}(t)=\text { the left-most extension of } \hat{t} \text { in } T \text { of length } \operatorname{lh}(t) \text {. }
$$

Then $\varrho_{T}$ is the identity on $T$, and the induced function $\boldsymbol{r}_{T}: \mathbb{R} \rightarrow[T]$ is a surjective Lipschitz function which is the identity on $[T]$, and it is called the canonical retraction of $\mathbb{R}$ onto $[T]$.

If a tree on $\omega$ is identified with its characteristic function, then the sets of all non-empty trees on $\omega$ can be identified with a closed subset of the Cantor space: if the $\boldsymbol{s}_{n}$ 's are as in (2), then every non-empty tree $T$ is coded by $x \in{ }^{\omega} 2$ where

$$
x(n)=1 \Longleftrightarrow \boldsymbol{s}_{n} \in T .
$$

In particular

$$
\lim _{n \rightarrow \infty} T_{n}=T \Longleftrightarrow \forall s \forall^{\infty} n\left(s \in T_{n} \Longleftrightarrow s \in T\right),
$$

where $\forall^{\infty}$ means "for all, but finitely many." Thus

$$
\mathrm{PTr}=\{T \mid T \text { is a pruned non-empty tree on } \omega\}
$$

is (identified with) a $\mathbf{G}_{\delta}$ subset of ${ }^{\omega} 2$. Similarly, the set

$$
\left\{\Phi \mid \operatorname{dom}(\Phi)=T \text { is a pruned tree on } \omega \text { and } \Phi: T \rightarrow^{<\omega} \omega \text { is Lipschitz }\right\}
$$

is a $\mathbf{G}_{\delta}$ subset of ${ }^{\omega} 2$, and therefore, if $\Phi: T \rightarrow{ }^{<\omega} \omega$ and $\Phi_{n}: T_{n} \rightarrow{ }^{<\omega} \omega(n \in \omega)$ are in this set, then

(7) $\lim _{n \rightarrow \infty} \Phi_{n}=\Phi \Longleftrightarrow \lim _{n \rightarrow \infty} T_{n}=T \& \forall s, t \forall^{\infty} n\left(\Phi_{n}(t)=s \Longleftrightarrow \Phi(t)=s\right)$.

In particular the set

$$
\boldsymbol{H}=\left\{\left\langle\left(T_{n}, \Phi_{n}\right) \mid n \in \omega\right\rangle \mid T_{n} \in \operatorname{PTr} \& \Phi_{n}: T_{n} \rightarrow{ }^{<\omega} \omega \text { is Lipschitz }\right\}
$$

is an uncountable Polish space. 


\section{The WAdge Hierarchy}

The results in this section, unless otherwise indicated, are either folklore or are taken from [Wad83]. We assume AD + DC $(\mathbb{R})$ throughout.

A. Basic facts. For $A, B \subseteq \mathbb{R}$ we say that $A$ is Wadge reducible to $B$, in symbols $A \leq_{\mathrm{W}} B$, just in case $A=f^{-1}$ " $B$ for some continuous $f: \mathbb{R} \rightarrow \mathbb{R}$; if the function $f$ is actually Lipschitz, then we will write $A \leq_{\mathrm{L}} B$. The relation $\leq_{\mathrm{W}}$ is a pre-order on $\mathscr{P}(\mathbb{R})$ and $\equiv_{\mathrm{W}}$ is the induced equivalence relation, $A \equiv_{\mathrm{W}} B$ iff $A \leq_{\mathrm{W}} B \& B \leq_{\mathrm{W}} A$. The equivalence classes modulo $\equiv_{\mathrm{W}}$ are called Wadge degrees, and

$$
[A]_{\mathrm{W}}=\left\{B \subseteq \mathbb{R} \mid B \equiv_{\mathrm{W}} A\right\}
$$

is the Wadge degree of $A$. The relation $A<_{\mathrm{W}} B$ means that $A \leq_{\mathrm{W}} B \& B \not_{\mathrm{W}} A$. The partial orders that $\leq_{\mathrm{W}}$ and $<_{\mathrm{W}}$ induce on the Wadge degrees are denoted by $\leq$ and $<$, that is $[A]_{\mathrm{W}} \leq[B]_{\mathrm{W}}$ iff $A \leq_{\mathrm{W}} B$ (and similarly for $<$ and $<_{\mathrm{W}}$ ). It is easy to see that $\emptyset \not_{\mathrm{W}} \mathbb{R}$ and $\mathbb{R} \not_{\mathrm{W}} \emptyset$, and that if $A \neq \emptyset, \mathbb{R}$ then $\emptyset<_{\mathrm{W}} A$ and $\mathbb{R}<_{\mathrm{W}} A$. In particular $[\emptyset]_{\mathrm{W}}=\{\emptyset\}$ and $[\mathbb{R}]_{\mathrm{W}}=\{\mathbb{R}\}$ are the $<$-least degrees. A set $A$-or a degree $[A]_{\mathrm{W}}$-is a successor if there is a set $B<_{\mathrm{W}} A$ such that for no set $C$ we have $B<_{\mathrm{W}} C<_{\mathrm{W}} A$. Otherwise it is said to be limit, unless $A=\mathbb{R}$ or $A=\emptyset$.

The Lipschitz game on $A, B \subseteq \mathbb{R}$, in symbols $G_{\mathrm{L}}(A, B)$, is the game where $\mathbf{I}$ and II alternately play natural numbers $a_{0}, b_{0}, a_{1}, b_{1}, \ldots$ and, letting $a=\left\langle a_{n} \mid n \in \omega\right\rangle$ and $b=\left\langle b_{n} \mid n \in \omega\right\rangle$, then

$$
\text { II wins } G_{\mathrm{L}}(A, B) \Longleftrightarrow(a \in A \Longleftrightarrow b \in B) \text {. }
$$

The Wadge game $G_{\mathrm{W}}(A, B)$ is similar, but II has the further option to pass at any given round. If II plays only finitely many times and passes from some point on, then he loses. Otherwise the winning condition is as before. It is not hard to see that

$$
\begin{aligned}
\text { II wins } G_{\mathrm{W}}(A, B) & \Longleftrightarrow A \leq_{\mathrm{W}} B \\
\text { II wins } G_{\mathrm{L}}(A, B) & \Longleftrightarrow A \leq_{\mathrm{L}} B
\end{aligned}
$$

where $A \leq_{\mathrm{L}} B$ means that $A$ is Wadge reducible to $B$ via a Lipschitz map. Moreover, if $\mathbf{I}$ wins $G_{\mathrm{L}}(A, B)$ or $G_{\mathrm{W}}(A, B)$, then $\neg B \leq_{\mathrm{L}} A$. Wadge's Lemma says that assuming $\mathrm{AD}$ (or even just the determinacy of all Lipschitz games) then

$$
A \leq_{\mathrm{W}} B \vee \neg B \leq_{\mathrm{W}} A .
$$

By a theorem of Martin's, $<_{\mathrm{W}}$ is well-founded on $\mathscr{P}(\mathbb{R})$ - see [Kec95, Thm 21.15] or [And03, Thm 5] for a proof. Therefore the sets of reals are stratified according to the ordinal $\|A\|_{\mathrm{W}}$, the Wadge rank of $A$, that is the rank of $A$ in the relation $<_{\mathrm{W}}$. For technical reasons, it is convenient to assume that the Wadge rank takes values in the non-zero ordinals, so that the $<_{W}$-least sets, $\mathbb{R}$ and $\emptyset$, have rank 1 . A set $A$ is Wadge self-dual (or simply: self-dual) iff $A \equiv_{\mathrm{W}} \neg A$, or, equivalently, $A \leq_{\mathrm{W}} \neg A$; otherwise $A$ is non-self-dual. A Wadge degree $[A]_{\mathrm{W}}$ is self-dual (non-self-dual) iff $A$ is self-dual (res. non-self-dual). A very useful result is the following

Theorem 2 (Steel, Van Wesep). If $A$ is non-self-dual and $B \leq_{\mathrm{W}} A$, then $B \leq_{\mathrm{L}} A$. 
For a proof see [VW78, Theorem 3.1].

This is how the structure of the Wadge degrees looks like:

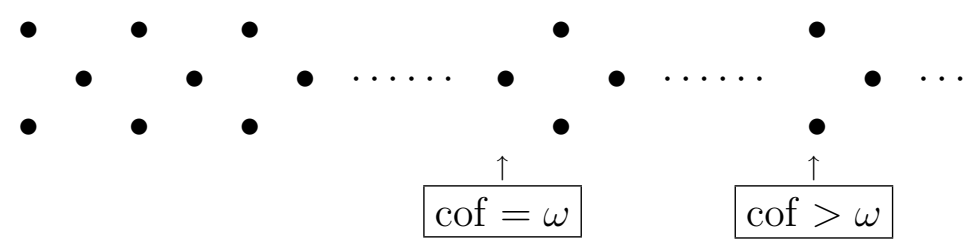

At limit levels of countable cofinality there is a single self-dual degree, at levels of uncountable cofinality there is a non-self-dual pair of degrees. The length of this hierarchy is the ordinal $\Theta$ defined in (1). Assuming $A D, \Theta$ is a cardinal, and moreover a fairly large one-larger than $\aleph_{1}, \aleph_{2}, \ldots, \aleph_{\omega}$, larger than the least $\aleph$-fixed point and so on. If we assume only a fragment of $A D$, say Borel determinacy, then the arguments and the picture above apply only to the Wadge degrees of Borel sets, and the length of the hierarchy is $\|A\|_{\mathrm{W}}$ where $A \in \Sigma_{1}^{1} \triangle \boldsymbol{\Pi}_{1}^{1}$, which is an ordinal strictly between $\omega_{1}$ and $\omega_{2}$.

As mentioned in the introduction, a pointclass is a non-empty $\Gamma \subset \mathscr{P}(\mathbb{R})$ closed under continuous pre-images. A pointclass $\Gamma$ is non-trivial if $\boldsymbol{\Gamma} \nsubseteq\{\emptyset, \mathbb{R}\}$; equivalent formulations are: $\boldsymbol{\Gamma}$ is infinite, or: $\boldsymbol{\Gamma} \supseteq \boldsymbol{\Delta}_{1}^{0}$. Since $<_{\mathrm{W}}$ is well-founded, then every pointclass $\boldsymbol{\Gamma}$ is of the form $\left\{A \subseteq \mathbb{R} \mid A<_{\mathrm{W}} B\right\}$ or of the form $\left\{A \subseteq \mathbb{R} \mid A \leq_{\mathrm{W}} B\right\}$ : in the latter case we say that $\Gamma$ is principal and generated by $A$, and the set $A$ (and hence any other $A^{\prime} \in[A]_{\mathrm{W}}$ ) is said to be $\boldsymbol{\Gamma}$-complete, or complete for $\boldsymbol{\Gamma}$. Not every pointclass is principal: for example $\boldsymbol{\Delta}_{1}^{1}$, the collection of Borel sets, is not. The dual of a pointclass $\Gamma$ is the pointclass $\{\neg A \mid A \in \Gamma\}$, and it is usually denoted by $\breve{\Gamma}$ or by $\Gamma^{\smile}$. A pointclass $\Gamma$ is self-dual if $\breve{\Gamma}=\Gamma$, that is, if it is closed under complements. Otherwise it is called non-self-dual. If $\Gamma$ is generated by $A$, then $\Gamma$ is self-dual iff $A$ is self-dual. Theorem 2 implies that

$$
\Gamma \text { non-self-dual and } A \in \Gamma \backslash \breve{\Gamma} \Longrightarrow \Gamma=\left\{X \mid X \leq_{\mathrm{L}} A\right\} \text {. }
$$

The non-principal pointclasses are of the form $\Gamma=\left\{B \subseteq \mathbb{R} \mid B<_{\mathrm{W}} A\right\}$, but not conversely: if $C$ is self-dual and $\|C\|_{\mathrm{W}}+1=\|A\|_{\mathrm{W}}$, then $\left\{B \subseteq \mathbb{R} \mid B<_{\mathrm{W}} A\right\}$ is generated by $C$. A non-self-dual pointclass must be principal, but, obviously, the converse is not true. The Wadge rank of a pointclass $\Gamma$ is

$$
\|\boldsymbol{\Gamma}\|_{\mathrm{W}}=\sup \left\{\|A\|_{\mathrm{W}}+1 \mid A \in \boldsymbol{\Gamma}\right\} .
$$

The +1 in the definition is needed to distinguish the Wadge rank of $\left\{X \mid X \leq_{\mathrm{W}} A\right\}$ from the one of $\left\{X \mid X<_{\mathrm{W}} A\right\}$, when $\|A\|_{\mathrm{W}}$ is limit. For example, if $\eta$ is the rank of a $\boldsymbol{\Sigma}_{1}^{1}$-complete or a $\boldsymbol{\Pi}_{1}^{1}$-complete set, then $\left\|\boldsymbol{\Delta}_{1}^{1}\right\|_{\mathrm{W}}=\eta$, while $\left\|\boldsymbol{\Sigma}_{1}^{1}\right\|_{\mathrm{W}}=\left\|\boldsymbol{\Pi}_{1}^{1}\right\|_{\mathrm{W}}=\eta+1$.

A level of the Wadge hierarchy is a set of the form

$$
\mathscr{P}^{(\alpha)}(\mathbb{R})=\left\{A \subseteq \mathbb{R} \mid\|A\|_{\mathrm{W}}<\alpha\right\}
$$

for some $\alpha$. It is a self-dual pointclass for all $\alpha>1$, and it is non-trivial if $\alpha>2$. Conversely, if $\|\boldsymbol{\Gamma}\|_{\mathrm{W}}=\alpha$ then $\boldsymbol{\Gamma} \cup \breve{\boldsymbol{\Gamma}}=\mathscr{P}^{(\alpha)}(\mathbb{R})$.

\section{B. Operations on degrees.}


B.1. The join operation and the tree $\boldsymbol{T}$. Given a sequence $A_{n} \subseteq \mathbb{R}$, the join of the $A_{n}$ 's is the set $\bigoplus_{n} A_{n}=\bigcup_{n} n^{\frown} A_{n}$. The join of $A$ and $B$ is $A \oplus B=\left(0^{\frown} A\right) \cup\left(1^{\frown} B\right)$. It is easy to check that $\left[\bigoplus_{n} A_{n}\right]_{\mathrm{W}}=\sup _{n}\left[A_{n}\right]_{\mathrm{W}}$, that is: $\forall i\left(\left[A_{i}\right]_{\mathrm{W}} \leq\left[\bigoplus_{n} A_{n}\right]_{\mathrm{W}}\right)$, and if $\forall i\left(\left[A_{i}\right]_{\mathrm{W}} \leq[B]_{\mathrm{W}}\right)$ then $\left[\bigoplus_{n} A_{n}\right]_{\mathrm{W}} \leq[B]_{\mathrm{W}}$. Similarly $[A \oplus B]_{\mathrm{W}}=\sup \left\{[A]_{\mathrm{W}},[B]_{\mathrm{W}}\right\}$.

For $A \subseteq \mathbb{R}$ and $\alpha \in$ Ord let

$$
\boldsymbol{T}(A ; \alpha)=\left\{s \in{ }^{<\omega} \omega \mid \alpha \leq\left\|A_{\lfloor s\rfloor}\right\|_{\mathrm{W}}\right\}
$$

and let

$$
\begin{aligned}
\boldsymbol{T}(A) & =\boldsymbol{T}\left(A ;\|A\|_{\mathrm{W}}\right) \\
& =\left\{s \in{ }^{<\omega} \omega \mid A_{\lfloor s\rfloor} \equiv_{\mathrm{W}} A\right\} .
\end{aligned}
$$

$\boldsymbol{T}(A ; \alpha)$ is a (possibly empty) tree on $\omega$. Since $(\neg A)_{\lfloor s\rfloor}=\neg\left(A_{\lfloor s\rfloor}\right)$ and since the Wadge rank of a set coincides with the rank of its complement, then $\boldsymbol{T}(A ; \alpha)=$ $\boldsymbol{T}(\neg A ; \alpha)$.

Lemma 3. If $B$ is self-dual and $\beta=\|B\|_{\mathrm{W}}$, then $\boldsymbol{T}(A ; \beta+1)$ is pruned.

Proof. If $\beta \geq\|A\|_{\mathrm{W}}$, then $\boldsymbol{T}(A ; \beta+1)=\emptyset$ hence the result is trivially true. So we may assume that $\beta<\|A\|_{\mathrm{W}}$ and hence that $\boldsymbol{T}(A ; \beta+1)$ is non-empty. Let $s \in \boldsymbol{T}(A ; \beta+1)$ and suppose that $\forall t \supset s\left(\left\|A_{\lfloor t\rfloor}\right\|_{\mathrm{W}} \leq \beta\right)$. Then $\forall n\left(A_{\left\lfloor s^{\wedge} n\right\rfloor} \leq{ }_{\mathrm{W}} B\right)$ and hence $A_{\lfloor s\rfloor}=\bigoplus_{n} A_{\left\lfloor s^{\wedge} n\right\rfloor} \leq_{\mathrm{W}} B$, a contradiction.

This implies that if $A$ is non-self-dual and $\|A\|_{\mathrm{W}}$ is a successor ordinal, then $\boldsymbol{T}(A)$ is pruned, since the immediate predecessor of a (successor) non-self-dual degree is self-dual. In fact Wadge showed that for each $A \subseteq \mathbb{R}$

$$
\begin{aligned}
A \text { is non-self-dual } & \Longleftrightarrow \boldsymbol{T}(A) \text { is pruned, } \\
A \text { is self-dual } & \Longleftrightarrow \boldsymbol{T}(A) \text { is well-founded. }
\end{aligned}
$$

B.2. The addition operation. The addition of $A, B \subseteq \mathbb{R}$ is the set

$$
A+B=\left\{(s+1)^{\frown} 0^{\frown} x \mid s \in{ }^{<\omega} \omega \& x \in A\right\} \cup\{x+1 \mid x \in B\} .
$$

The following are easily proven:

(11) $\neg(A+B)=\neg A+\neg B$,

(12) $A \leq_{\mathrm{W}} A^{\prime} \& B \leq_{\mathrm{W}} B^{\prime} \Longrightarrow A+B \leq_{\mathrm{W}} A^{\prime}+B^{\prime}$.

(13) $A+B_{\lfloor s\rfloor}=(A+B)_{\lfloor s+1\rfloor}$.

Lemma 4. Let $A$ be self-dual.

(a) $\left([A+\emptyset]_{\mathrm{W}},[A+\mathbb{R}]_{\mathrm{W}}\right)$ is the least non-self-dual pair above $[A]_{\mathrm{W}}$. In particular $A<\mathrm{W} A+B$ for any $B$.

(b) $B \leq_{\mathrm{W}} C \Longleftrightarrow A+B \leq_{\mathrm{W}} A+C$. 
Proof. (a) Since $\neg(A+\mathbb{R}) \equiv_{\mathrm{W}} A+\emptyset$ by (11) and (12), and $\boldsymbol{T}(A+\emptyset)=\boldsymbol{T}(A+$ $\mathbb{R})=\left\{s+1 \mid s \in{ }^{<\omega} \omega\right\}$ is ill-founded, then $\left([A+\emptyset]_{\mathrm{W}},[A+\mathbb{R}]_{\mathrm{W}}\right)$ is a non-selfdual pair by (10). Suppose $A<_{\mathrm{W}} B$ and $B$ is non-self-dual, so that $\boldsymbol{T}(B)$ is ill-founded by (10): we will show that $[\boldsymbol{T}(B)] \cap B \neq \emptyset \Longrightarrow A+\mathbb{R} \leq_{\mathrm{W}} B$ and $[\boldsymbol{T}(B)] \cap \neg B \neq \emptyset \Longrightarrow A+\emptyset \leq_{\mathrm{W}} B$. Since $A \leq_{\mathrm{W}} A+\emptyset$ and $A \leq_{\mathrm{W}} A+\mathbb{R}$ via the function $x \mapsto 0^{\frown} x$, this will finish the proof. Suppose $b \in[\boldsymbol{T}(B)] \cap B$ : then II wins $G_{\mathrm{W}}(A+\mathbb{R}, B)$ by enumerating $b$ as long as $\mathbf{I}$ does not play 0 . If at some round $n \mathbf{I}$ plays 0 then II can win the game from this point on since $A \leq_{\mathrm{W}} B_{\lfloor b \mid n\rfloor}$. This proves that $[\boldsymbol{T}(B)] \cap B \neq \emptyset \Longrightarrow A+\mathbb{R} \leq_{\mathrm{W}} B$. The other implication, being similar, is left to the reader.

(b) The $(\Longrightarrow)$ direction follows from $(12)$, so it is enough to prove $(\Longleftarrow)$. Suppose $\tau$ is a winning strategy for II in $G_{\mathrm{W}}(A+B, A+C)$. It is enough to show that II is not the first player to play 0 , since then II wins $G_{\mathrm{W}}(B, C)$ using $\tau^{\prime}$ defined by

$$
\tau^{\prime}(s)=n \Longleftrightarrow \tau(s+1)=n+1 \text {. }
$$

Towards a contradiction suppose $\tau(s+1)=0$, for some $s \in{ }^{<\omega} \omega$ of minimal length. Let also $t+1$ be the sequence constructed by $\tau$ before this stage, so that after this round the two positions will be $s+1$ and $(t+1)^{\frown} 0$. Then

$$
\begin{aligned}
A & <\mathrm{W} A+B_{\lfloor s\rfloor} & & \text { (by part (a)) } \\
& =(A+B)_{\lfloor s+1\rfloor} & & \text { (by }(13)) \\
& \leq{ }_{\mathrm{W}}(A+C)_{\lfloor(t+1)-0\rfloor} & & \text { (since } \tau \text { is winning) } \\
& =A, & &
\end{aligned}
$$

a contradiction.

The hypothesis in (a) that $A$ be self-dual is necessary since, for example, $\mathbb{R}+\mathbb{R}=$ $\mathbb{R}$. Thus if $A$ is self-dual, then

$$
A+B<_{\mathrm{W}} A+C \Longleftrightarrow B<_{\mathrm{W}} C \text {. }
$$

In other words, the map $B \mapsto A+B$ is strictly $<{ }_{\mathrm{W}}$-increasing, hence $\|A\|_{\mathrm{w}}+\|B\|_{\mathrm{W}} \leq$ $\|A+B\|_{\mathrm{w}}$. In fact equality holds, that is:

(14) if $A$ is self-dual and $B$ is arbitrary then $\|A+B\|_{\mathrm{W}}=\|A\|_{\mathrm{W}}+\|B\|_{\mathrm{W}}$.

To prove this it is enough to show that if $A<_{\mathrm{W}} B$ with $A$ self-dual, then $B \equiv_{\mathrm{W}} A+C$, for some set $C$, and this is the content of part (a) of the next result.

Lemma 5. Suppose $A<_{\mathrm{W}} B$, with $A$ self-dual and let $\alpha=\|A\|_{\mathrm{W}}$. Let $T=$ $\boldsymbol{T}(B ; \alpha+1)$ and let $\boldsymbol{r}_{T}: \mathbb{R} \rightarrow[T]$ be the canonical retraction of $\mathbb{R}$ onto $[T]$. Then:

(a) $A+\boldsymbol{r}_{T}^{-1}$ " $(B \cap[T]) \equiv_{\mathrm{W}} B$.

(b) $\|B \backslash[T]\|_{\mathrm{W}} \leq \alpha+1$.

(c) Suppose there is a self-dual set of Wadge rank $\alpha+\gamma$, and $\alpha+\gamma+1 \leq\|B\|_{\mathrm{W}}$. Let $U=\boldsymbol{T}(B ; \alpha+\gamma+1)$. Then $U \subseteq T$ and

$$
\| \boldsymbol{r}_{T}^{-1} \text { “ }(B \cap([T] \backslash[U])) \|_{\mathrm{W}} \leq \gamma+1 .
$$


Proof. (a) First of all, notice that the tree $T$ is pruned by Lemma 3, so that the maps $\varrho_{T}:<\omega \omega \rightarrow T$ as in (4) and $\boldsymbol{r}_{T}: \mathbb{R} \rightarrow[T]$ are defined. For notational simplicity, let $C=\boldsymbol{r}_{T}^{-1}$ " $(B \cap[T])$. Then II wins $G_{\mathrm{W}}(A+C, B)$ as follows:

As long as $\mathbf{I}$ does not play 0 , then II subtracts 1 and applies $\varrho_{T}$ to maintain his position inside $T$, so that if at round $n \mathbf{I}$ is at position $s+1$, then II is at position $\varrho_{T}(s)$. Thus if $\mathbf{I}$ never plays 0 , then

$$
x+1 \in A+C \Longleftrightarrow x \in C \Longleftrightarrow \boldsymbol{r}_{T}(x) \in B .
$$

If $\mathbf{I}$ plays 0 at round $n$, then $\mathbf{I I}$ passes and then applies a reduction of $A$ to $B_{\left\lfloor\varrho_{T}(s)\right\rfloor}$, where $s$ is I's position before round $n$. Such a reduction exists since $\varrho_{T}(s) \in T$, and therefore $\|A\|_{\mathrm{W}}<\left\|B_{\left.\varrho_{T}(s)\right\rfloor}\right\|_{\mathrm{W}}$.

Conversely II wins $G_{\mathrm{W}}(B, A+C)$ as follows:

As long as I plays inside $T$ then II simply copies I's moves and adds 1 , so that the respective positions will be $s \in T$ and $s+1$. Thus if $\mathbf{I}$ never leaves $T$, letting $x$ and $x+1$ be the reals played by $\mathbf{I}$ and II, then $\boldsymbol{r}_{T}(x)=x \in[T]$ and

$$
x \in B \Longleftrightarrow x \in C \Longleftrightarrow x+1 \in A+C .
$$

If at some round $\mathbf{I}$ reaches a position $s \in \partial T$, then II plays 0 and then, since $B_{\lfloor s\rfloor} \leq_{\mathrm{W}} A, \mathbf{I I}$ can apply a reduction of $B_{\lfloor s\rfloor}$ to $A$ and win the game.

Therefore $A+C \equiv_{\mathrm{W}} B$ as required.

We now prove part (b). By part (a) of Lemma $4,\|A+\emptyset\|_{\mathrm{W}}=\|A\|_{\mathrm{W}}+1$, so it is enough to prove that II wins $G_{\mathrm{W}}(B \backslash[T], A+\emptyset)$. Consider the following strategy for II:

As long as I plays inside $T$ then II plays 1 . If at some round $\mathbf{I}$ reaches a position $s \in \partial T$, then $\mathbf{I I}$ answers 0 and then applies a reduction of $B\lfloor s\rfloor$ to $A$.

It is clear that this is a winning strategy for II, hence (b) holds.

Finally we deal with part (c). The reduction in part (a) witnessing $A+\boldsymbol{r}_{T}^{-1}$ " $(B \cap$ $[T]) \leq_{\mathrm{W}} B$ proves that

$$
A+\boldsymbol{r}_{T}^{-1} \text { “ }(B \cap([T] \backslash[U])) \leq_{\mathrm{W}} B \backslash[U] .
$$

By part (b) with $U$ replacing $T$ we have that $\|B \backslash[U]\|_{\mathrm{w}} \leq \alpha+\gamma+1$. Therefore by (14) — which follows from part (a) - we have

$$
\|A\|_{\mathrm{W}}+\| \boldsymbol{r}_{T}^{-1} \text { “ }(B \cap([T] \backslash[U])) \|_{\mathrm{W}} \leq \alpha+\gamma+1,
$$

hence $\| \boldsymbol{r}_{T}^{-1}$ “ $(B \cap([T] \backslash[U])) \|_{\mathrm{W}} \leq \gamma+1$.

Part (c) of Lemma 5 will be useful in section 4.

Lemma 6. (a) If $C$ is closed and $U$ is open, then $A \cap C \leq_{\mathrm{W}} \emptyset+A$ and $A \cup U \leq_{\mathrm{W}}$ $\mathbb{R}+A$. In particular, if $\|A\|_{\mathrm{W}} \geq \omega$, then $A \cap C \leq_{\mathrm{W}} A$ and $A \cup U \leq_{\mathrm{W}} A$.

(b) If $\|A\|_{\mathrm{W}} \geq \omega$, then $A \backslash \operatorname{Int}(A) \equiv_{\mathrm{W}} A$. 
(c) If $\|\boldsymbol{\Gamma}\|_{\mathrm{W}} \geq \omega$, then $\boldsymbol{\Gamma}$ is closed under intersections with closed sets and under unions with open sets.

Proof. (a) If $T$ is a pruned tree on $\omega$, then II wins $G_{\mathrm{W}}(A \cap[T], \emptyset+A)$ :

As long as I plays in $T$, then II copies (and adds 1 ); if $\mathbf{I}$ leaves $T$ then II plays 0 and copies.

We leave it to the reader to check that this is a winning strategy.

If $\|A\|_{\mathrm{W}} \geq \omega$ and $D \in \Delta_{1}^{0} \backslash\{\mathbb{R}, \emptyset\}$, then

$$
\|\emptyset+A\|_{\mathrm{W}} \leq\|D+A\|_{\mathrm{W}}=2+\|A\|_{\mathrm{W}}=\|A\|_{\mathrm{W}},
$$

and since $A \leq_{\mathrm{W}} \emptyset+A$, then $A \equiv_{\mathrm{W}} \emptyset+A$, which implies the desired conclusion.

The case of $A \cup U$ with $U$ open, can be obtained by taking complements.

(b) Let $B=A \backslash \operatorname{Int}(A)$. By part (a) $B \leq_{\mathrm{W}} A$ and $A=B \cup \operatorname{Int}(A) \leq_{\mathrm{W}} \mathbb{R}+B$. Since $\|A\|_{\mathrm{W}} \leq\|\mathbb{R}+B\|_{\mathrm{W}} \leq\|\mathbb{R}+A\|_{\mathrm{W}}=\|A\|_{\mathrm{W}}$, then $B \equiv_{\mathrm{W}} A$ follows.

(c) Follows at once from part (a).

If $A=f^{-1}$ " $B$, where $f:{ }^{\omega_{2}} \rightarrow \mathbb{R}$ is continuous, then it does not necessarily follow ${ }^{1}$ that $A \leq_{\mathrm{W}} B$ (take, e.g., ${ }^{\omega} 2, \mathbb{R}$, and the inclusion function).

Lemma 7. Suppose $A \subseteq{ }^{\omega} 2, B \subseteq \mathbb{R}$ and $\|B\|_{\mathrm{W}} \geq \omega$. Suppose also $A=f^{-1}$ " $B$, where $f:{ }^{\omega} 2 \rightarrow \mathbb{R}$ is continuous. Then $A \leq_{\mathrm{w}} B$.

Proof. By part (b) of Lemma 6, we may assume that $B$ has empty interior. Let $\Phi:{ }^{<\omega} 2 \rightarrow{ }^{<\omega} \omega$ be a continuous map inducing $f$. Since, by assumption, $B_{\lfloor s\rfloor} \neq \mathbb{R}$ for all $s$, choose a real $b_{s} \in \mathbb{R} \backslash B_{\lfloor s\rfloor}$. Let $\Psi:{ }^{<\omega} \omega \rightarrow{ }^{<\omega} \omega$ be defined as follows: for every $t \in{ }^{<\omega} \omega$ let $\hat{t} \subseteq t$ be largest such that $\hat{t} \in{ }^{<\omega} 2$ and let $n=\operatorname{lh}(t)-\operatorname{lh}(\hat{t})$ and let

$$
\Psi(t)=\Phi(\hat{t})^{\frown}\left(b_{\Phi(\hat{t})} \uparrow n\right) .
$$

It is easy to check that $\Psi$ is continuous and that its induced function witnesses $A \leq_{\mathrm{W}} B$.

B.3. The $\downarrow$ and $b$ operations. For $A \subseteq \mathbb{R}$ let

$$
A^{\natural}=\left\{\left(s_{0}+1\right)^{\frown} 0^{\frown} \ldots \frown 0^{\frown}\left(s_{n}+1\right)^{\frown} 0^{\frown}(x+1) \mid s_{0}, \ldots, s_{n} \in{ }^{<\omega} \omega \& x \in A\right\}
$$

and let $A^{\natural}=A^{\natural} \cup\left\{x \in \mathbb{R} \mid \exists^{\infty} n x(n)=0\right\}$, where in the definition of $A^{\natural}$ it is understood that its elements might be of the form $x+1$ with $x \in A$. The $\downarrow$ and $b$ operations enjoy the following properties:

(15) $A \leq_{\mathrm{W}} B \Longrightarrow A \leq_{\mathrm{W}} A^{\natural} \leq_{\mathrm{W}} B^{\natural} \& A \leq_{\mathrm{W}} A^{b} \leq_{\mathrm{W}} B^{b}$ (monotonicity).

(16) $A^{\text {虾 }} \equiv_{\mathrm{W}} A^{\natural}$ and $A^{\text {bb }} \equiv_{\mathrm{W}} A^{\mathrm{b}}$ (idempotence).

(17) If $A$ is self-dual, then $\left[A^{\natural}\right]_{\mathrm{W}},\left[A^{b}\right]_{\mathrm{W}}$ are a non-self-dual pair, and

$$
\left\|A^{\natural}\right\|_{\mathrm{W}}=\|A\|_{\mathrm{W}} \cdot \omega_{1} \text {. }
$$

\footnotetext{
${ }^{1}$ Recall that, by definition, a function witnessing $A \leq_{\mathrm{W}} B$ must have domain $\mathbb{R}$.
} 
(18) $\emptyset^{\natural}=\emptyset$ and $\mathbb{R}^{\natural}=\left\{x \in \mathbb{R} \mid \forall^{\infty} n x(n) \neq 0\right\} \in \Sigma_{2}^{0} \backslash \Pi_{2}^{0}$, hence by monotonicity and idempotence

$$
\forall A \in \Sigma_{2}^{0} \backslash\{\emptyset\}\left(A^{\natural} \equiv_{\mathrm{W}} \mathbb{R}^{\natural}\right) .
$$

In particular, if $A$ is clopen, $\left\|\mathbb{R}^{\natural}\right\|_{\mathrm{W}}=\left\|A^{\natural}\right\|_{\mathrm{W}}=2 \cdot \omega_{1}=\omega_{1}$.

Similarly, $\mathbb{R}^{b}=\mathbb{R}, \emptyset^{b}=\left\{x \in \mathbb{R} \mid \exists^{\infty} n x(n)=0\right\} \in \Pi_{2}^{0} \backslash \Sigma_{2}^{0}, \forall A \in$ $\Pi_{2}^{0} \backslash\{\mathbb{R}\}\left(A^{b} \equiv_{\mathrm{W}} \emptyset^{b}\right)$ and $\left\|\emptyset^{b}\right\|_{\mathrm{W}}=\omega_{1}$.

If $\|A\|_{\mathrm{W}}$ is a successor ordinal or limit of cofinality $\omega$, then $A^{\natural}$ is non-self-dual and $\left\|A^{\natural}\right\|_{\mathrm{W}}=\|A\|_{\mathrm{W}} \cdot \omega_{1}$ : this is immediate by (17) if $\|A\|_{\mathrm{W}}$ is limit of cofinality $\omega$ or if $\|A\|_{\mathrm{W}}$ is a successor and $A$ is self-dual; if $A$ is non-self-dual and $\|B\|_{\mathrm{W}}+1=\|A\|_{\mathrm{W}}$, then by monotonicity $B^{\natural} \leq_{\mathrm{W}} A^{\natural} \leq_{\mathrm{W}}(A \oplus \neg A)^{\natural}$, hence

$$
\left\|B^{\natural}\right\|_{\mathrm{W}}=\left(\|A\|_{\mathrm{W}}-1\right) \cdot \omega_{1}=\|A\|_{\mathrm{W}} \cdot \omega_{1}=\left(\|A\|_{\mathrm{W}}+1\right) \cdot \omega_{1}=\left\|(A \oplus \neg A)^{\natural}\right\|_{\mathrm{W}},
$$

so that $B^{\natural} \equiv_{\mathrm{W}} A^{\natural} \equiv_{\mathrm{W}}(A \oplus \neg A)^{\natural}$, which implies the desired result. On the other hand, if $\|A\|_{\mathrm{W}}$ is limit of uncountable cofinality, then $\left\|A^{\natural}\right\|_{\mathrm{W}}$ is either $\|A\|_{\mathrm{W}}$ or $\|A\|_{\mathrm{W}} \cdot \omega_{1}$ : if $\left\|A^{\natural}\right\|_{\mathrm{W}} \neq\|A\|_{\mathrm{W}}$, then $\|A\|_{\mathrm{W}}<\left\|A^{\natural}\right\|_{\mathrm{W}}$ since $A \leq_{\mathrm{W}} A^{\natural}$ by $(15)$, and as $A<_{\mathrm{W}} A \oplus \neg A \leq_{\mathrm{W}} A^{\natural}$, then by monotonicity and idempotence $(A \oplus \neg A)^{\natural} \equiv_{\mathrm{W}} A^{\natural}$; since $A \oplus \neg A$ is self-dual, then $\left\|A^{\natural}\right\|_{\mathrm{W}}=\|A \oplus \neg A\|_{\mathrm{W}} \cdot \omega_{1}=\|A\|_{\mathrm{W}} \cdot \omega_{1}$. To see that both possibilities $\left(\left\|A^{\natural}\right\|_{\mathrm{W}}=\|A\|_{\mathrm{W}}\right.$ and $\left.\left\|A^{\natural}\right\|_{\mathrm{W}}=\|A\|_{\mathrm{W}} \cdot \omega_{1}\right)$ can occur, consider for the first case $A=\mathbb{R}^{\natural}$, and $A=\emptyset^{b}$ for the second. The first case is immediate from (16) and for the second notice that $\mathbb{R} \leq_{W} \emptyset^{b} \leq_{W}\left(\emptyset^{b}\right)^{\natural}$ hence $\mathbb{R}^{\natural} \leq_{W}\left(\emptyset^{b}\right)^{\natural}$ : if $\left(\emptyset^{b}\right)^{\natural}$ and $\emptyset^{b}$ were Wadge equivalent, then $\mathbb{R}^{\natural} \leq_{\mathrm{W}} \emptyset^{b}$, contrary to the fact that $\left[\emptyset^{b}\right]_{\mathrm{W}},\left[\mathbb{R}^{\natural}\right]_{\mathrm{W}}$ form a non-self-dual pair by (17) and (18), and therefore

$$
\|A\|_{\mathrm{W}}=\left\|\emptyset^{b}\right\|_{\mathrm{W}}=\left\|\mathbb{R}^{\natural}\right\|_{\mathrm{W}}<\left\|\left(\emptyset^{b}\right)^{\natural}\right\|_{\mathrm{W}}=\left\|A^{\natural}\right\|_{\mathrm{W}} .
$$

The $\downarrow$ and $b$ operations can be used to construct canonical sets of rank $\omega_{1}^{n}$. Let

$$
\mathrm{C}_{0}=\emptyset, \quad \mathrm{C}_{n+1}=\left(\neg \mathrm{C}_{n}\right)^{\natural}, \quad \mathrm{C}_{\omega}=\bigoplus_{n} \mathrm{C}_{n} .
$$

Then $\neg \mathrm{C}_{n+1} \equiv_{\mathrm{W}} \mathrm{C}_{n}^{b},\left\|\mathrm{C}_{n}\right\|_{\mathrm{W}}=\omega_{1}^{n}$, and $\left\|\mathrm{C}_{\omega}\right\|_{\mathrm{W}}=\omega_{1}^{\omega}$. Say that a pointclass $\boldsymbol{\Gamma}$ is ฤ-closed iff $A \in \Gamma \Longrightarrow A^{\natural} \in \Gamma$. The -closed pointclasses are most important, since these pointclasses (and their duals) are exactly those $\Gamma^{\prime}$ 's such that $|\boldsymbol{\Gamma}|>\left|\boldsymbol{\Gamma}^{\prime}\right|$, for all $\Gamma^{\prime} \subset \Gamma$, as we shall see in the next section.

By (15) and (16) the first $\omega+1$--closed pointclasses are

$$
\begin{aligned}
\boldsymbol{\Lambda}_{n} & =\left\{A \mid A \leq_{\mathrm{W}} \mathrm{C}_{n}\right\} \quad(n \in \omega) \\
\boldsymbol{\Lambda}_{\omega} & =\left\{A \mid A<{ }_{\mathrm{W}} \mathrm{C}_{\omega}\right\} .
\end{aligned}
$$

Then $\boldsymbol{\Lambda}_{0} \subset \boldsymbol{\Lambda}_{1} \subset \ldots$ are non-self-dual, $\boldsymbol{\Lambda}_{n}$ non-trivial if $n>0$, and $\left\|\boldsymbol{\Lambda}_{n}\right\|_{\mathrm{W}}=\omega_{1}^{n}+1$, while $\boldsymbol{\Lambda}_{\omega}$ is self-dual and $\left\|\boldsymbol{\Lambda}_{\omega}\right\|_{\mathrm{W}}=\omega_{1}^{\omega}$. Since $\left\{B \mid B \leq_{\mathrm{W}} A^{\natural}\right\}$ is always a $\downarrow$-closed pointclass by (15) and (16), then there is a non-self-dual - and hence principalpointclass of Wadge rank $\omega_{1}^{\xi+1}+1$, for every $\xi<\Theta$. On the other hand $\mathscr{P}^{\left(\omega_{1}^{\xi+1}\right)}(\mathbb{R})$, the self-dual pointclass of rank $\omega_{1}^{\xi+1}$, is not $\sharp$-closed, since if $A$ is a self-dual set of minimal rank $\geq \omega_{1}^{\xi}$, then $A \in \mathscr{P}^{\left(\omega_{1}^{\xi+1}\right)}(\mathbb{R})$ but $\left\|A^{\natural}\right\|_{\mathrm{W}}=\omega_{1}^{\xi+1}$ hence $A^{\natural} \notin \mathscr{P}^{\left(\omega_{1}^{\xi+1}\right)}(\mathbb{R})$. 
As the increasing union of $\downarrow$-closed pointclasses is $\downarrow$-closed, there are self-dual nonprincipal $\downarrow$-closed pointclasses of rank $\omega_{1}^{\xi}$ with $\xi$ limit. The calculations above leave open the case of a non-self-dual pair of pointclasses $\Gamma, \breve{\Gamma}$ of $\operatorname{rank} \omega_{1}^{\xi}+1$, with $\xi$ limit of uncountable cofinality. This case was settled by Van Wesep in [VW77], by showing that at least one among $\boldsymbol{\Gamma}$ and $\breve{\Gamma}$ is $\downarrow$-closed.

To summarize:

(21) Let $\boldsymbol{\Gamma}$ be $\measuredangle$-closed: if $\boldsymbol{\Gamma}$ is non-self-dual, then $\|\boldsymbol{\Gamma}\|_{\mathrm{W}}=\omega_{1}^{\xi}+1$; if $\boldsymbol{\Gamma}$ is self-dual, then $\|\boldsymbol{\Gamma}\|_{\mathrm{w}}=\omega_{1}^{\xi}$ with $\xi$ limit.

Conversely: if $\boldsymbol{\Gamma}$ is self-dual and $\|\boldsymbol{\Gamma}\|_{\mathrm{W}}=\omega_{1}^{\xi}$ with $\xi$ limit, then $\boldsymbol{\Gamma}$ is -tclosed; if $\boldsymbol{\Gamma}$ is non-self-dual and $\|\boldsymbol{\Gamma}\|_{\mathrm{W}}=\omega_{1}^{\xi}+1$, then

- if $\xi$ is a successor ordinal, then exactly one among $\boldsymbol{\Gamma}$ and $\breve{\Gamma}$ is $t$-closed,

- if $\xi$ is a limit ordinal and $\operatorname{cof}(\xi)>\omega$, then at least one among $\boldsymbol{\Gamma}$ and $\breve{\Gamma}$ is $\natural$-closed.

The next two results characterize the $\downarrow$-closed pointclasses.

Lemma 8. Let $\boldsymbol{\Gamma}$ be a pointclass such that whenever $A_{n} \in \boldsymbol{\Gamma}$ and $F_{n} \in \boldsymbol{\Pi}_{1}^{0}$, with $F_{n} \cap F_{m}=\emptyset$ for $n \neq m$, then $\bigcup_{n}\left(F_{n} \cap A_{n}\right) \in \boldsymbol{\Gamma}$. Then $\boldsymbol{\Gamma}$ is $\natural$-closed.

Proof. Let $A \in \boldsymbol{\Gamma}$. Since $\emptyset^{\natural}=\emptyset \in \boldsymbol{\Gamma}$, we may assume that $A \neq \emptyset$. Let $\left\langle s_{n} \mid n \in \omega\right\rangle$ be an enumeration without repetitions of

$$
\{\emptyset\} \cup\left\{s^{\frown} 0 \mid s \in{ }^{<\omega} \omega\right\} .
$$

Let

$$
\begin{aligned}
& \left.F_{n}=\left\{s_{n}\right\urcorner(x+1) \mid x \in \mathbb{R}\right\}, \\
& \left.A_{n}=\left\{s_{n}\right\urcorner y \mid y \dot{-} 1 \in A\right\} .
\end{aligned}
$$

Then $A_{n} \cap F_{n}=A^{\natural} \cap F_{n}$, and since $A^{\natural} \subseteq \bigcup_{n} F_{n}$, then $A^{\natural}=\bigcup_{n}\left(A_{n} \cap F_{n}\right)$. The $F_{n}$ 's are $\boldsymbol{\Pi}_{1}^{0}$ and they are pairwise disjoint (since the $s_{n}$ 's are distinct), so we only need to check that $A_{n} \in \boldsymbol{\Gamma}$. In fact each $A_{n}$ is Wadge reducible to $A$ via the function

$$
x \mapsto \begin{cases}y \dot{-} 1 & \text { if } x=s_{n}^{\curlyvee} y, \\ a & \text { if } s_{n} \not \subset x,\end{cases}
$$

where $a \notin A$.

Conversely,

Lemma 9. Suppose $\boldsymbol{\Gamma}$ is generated by $A$, and that it is $F_{n} \in \Pi_{1}^{0}$, with $F_{n} \cap F_{m}=\emptyset$ if $n \neq m$, then $\bigcup_{n}\left(F_{n} \cap A_{n}\right) \in \Gamma$.

Proof. Say $C=\bigcup_{n}\left(F_{n} \cap A_{n}\right)$ with $A_{n} \in \boldsymbol{\Gamma}$ and with $F_{n} \in \boldsymbol{\Pi}_{1}^{0}$ and disjoint. We must show that $C \leq_{\mathrm{W}} A^{\natural}$. Let $T_{n}$ be the pruned tree such that $F_{n}=\left[T_{n}\right]$, and for each $n$ fix a winning strategy $\tau_{n}$ for II in $G_{\mathrm{W}}\left(A_{n}, A\right)$. Then II wins $G_{\mathrm{W}}\left(C, A^{\natural}\right)$ as follows: 
II follows $\tau_{0}+1$ as long as I's position is in $T_{0}$;

if $\mathbf{I}$ leaves $T_{0}$, then II plays 0 and switches to $\tau_{1}+1$ and follows it as long as I's position is in $T_{1}$;

if $\mathbf{I}$ leaves $T_{1}$, then II plays 0 and switches to $\tau_{2}+1$ and follows it as long as I's position is in $T_{3}$;

.... And so on.

Let $x$ and $y$ be the reals played by $\mathbf{I}$ and II according to the strategy above. If $x \notin \bigcup_{n}\left[T_{n}\right]$ then $y$ will contain infinitely many 0 's so $x \notin C$ and $y \notin A^{\natural}$, hence II wins. If instead $x \in\left[T_{n}\right]$ for some $n$, then after $n$-many false starts II will settle on the strategy $\tau_{n}$ so that $y=s^{\frown}(z+1)$ where $s$ is a finite sequence containing $n$-many 0's including one in its last entry, and $z$ is $\tau_{n}$ 's response to $x$. Therefore $x \in C \Longleftrightarrow x \in A_{n} \Longleftrightarrow z \in A \Longleftrightarrow y \in A^{\natural}$, and II wins.

Corollary 10. The smallest $\downarrow$-closed pointclass containing a set $A$ is

$$
\left\{\bigcup_{n}\left(F_{n} \cap A_{n}\right) \mid F_{n} \in \Pi_{1}^{0} \text { are pairwise disjoint and } A_{n} \leq_{\mathrm{W}} A\right\} .
$$

The hypothesis in Lemma 9 that $\boldsymbol{\Gamma}$ is principal is necessary, since if each $\boldsymbol{\Gamma}_{n}$ is দ-closed and $\boldsymbol{\Gamma}_{0} \subset \boldsymbol{\Gamma}_{1} \subset \ldots$, then $\boldsymbol{\Gamma}_{\infty}=\bigcup_{n} \boldsymbol{\Gamma}_{n}$ does not satisfy the conclusion of Lemma 9. To see this, for each $n$ pick $B_{n} \in \boldsymbol{\Gamma}_{n}$ with $B_{n+1} \notin \boldsymbol{\Gamma}_{n}$, so that $\bigoplus_{n} B_{n} \notin \boldsymbol{\Gamma}_{\infty}$ : then each $A_{n}=n^{\frown} B_{n}$ is in $\boldsymbol{\Gamma}_{\infty}$, the $F_{n}=n^{\frown} \mathbb{R}$ are closed (in fact: clopen) and pairwise disjoint, and $\bigoplus_{n} B_{n}=\bigcup_{n}\left(F_{n} \cap A_{n}\right)$.

Also notice that since every $\boldsymbol{\Sigma}_{2}^{0}$ in the Baire space is the countable union of disjoint closed sets, then the $F_{n}$ 's in the results above can be replaced with disjoint $\Sigma_{2}^{0}$ sets.

By Lemmas 6, 8 and 9 , if $\boldsymbol{\Gamma}$ is principal and $\|\boldsymbol{\Gamma}\|_{\mathrm{W}} \geq \omega$, then

$$
\left\{\bigcup_{n} A_{n} \mid A_{n} \in \boldsymbol{\Gamma} \text { and the } \mathrm{Cl}\left(A_{n}\right) \text { are pairwise disjoint }\right\}
$$

is the least $\downarrow$-closed pointclass containing $\Gamma$.

B.4. The difference hierarchy. Recall Hausdorff's definition of the difference hierarchy over a pointclass $\Gamma$ : Given an $n$-tuple $\left\langle A_{0}, \ldots, A_{n-1}\right\rangle$ of subsets of $\mathbb{R}$ let

$$
\operatorname{Diff}_{k<n} A_{k}= \begin{cases}\bigcup_{2 k<n}\left(A_{2 k} \backslash \bigcup_{i<2 k} A_{i}\right) & \text { if } n \text { is odd, } \\ \bigcup_{2 k+1<n}\left(A_{2 k+1} \backslash \bigcup_{i<2 k+1} A_{i}\right) & \text { if } n \text { is even. }\end{cases}
$$

In other words, $\operatorname{Diff}_{k<n} A_{k}=\left\{x \in \bigcup_{k<n} A_{k} \mid\right.$ the least $k<n$ such that $x \in A_{k}$ has parity different from $n\}$, and let

$$
n-\boldsymbol{\Gamma}=\left\{\operatorname{Diff}_{k<n} A_{k} \mid A_{0}, A_{1}, \ldots, A_{n-1} \in \Gamma\right\}
$$

be the pointclass of all $n$-differences of sets in $\Gamma$. (In the literature $n$ - $\Gamma$ is often denoted by $\operatorname{Diff}(n ; \boldsymbol{\Gamma})$.) Clearly $1-\boldsymbol{\Gamma}$ is simply $\boldsymbol{\Gamma}$. (These definitions extend to the case of $n$ a non-zero countable ordinal.)

Suppose $\boldsymbol{\Gamma}$ is a pointclass closed under finite unions. Since $\operatorname{Diff}_{k<n}\left(\bigcup_{i<k} A_{i}\right)=$ $\operatorname{Diff}_{k<n} A_{k}$, it follows that $n$ - $\boldsymbol{\Gamma}$ is the set of all $n$-differences of increasing $n$-tuples of sets in $\Gamma$.

For any fixed $n$, letting $\hat{A}_{k}=A_{n-1-k}$ for $k<n$ and $\hat{A}_{n}=\emptyset$, defines a bijection

$$
\left\langle A_{0}, \ldots, A_{n-1}\right\rangle \mapsto\left\langle\hat{A}_{0}, \ldots, \hat{A}_{n}\right\rangle
$$


between the family of all increasing $n$-tuples of sets in $\Gamma$ and the family of all decreasing $n+1$-tuples of sets in $\Gamma$ with last set empty. Regardless wether $n$ is even or odd we have that if $A_{0} \subseteq A_{1} \subseteq \cdots \subseteq A_{n-1}$ then

$$
\operatorname{Diff}_{k<n} A_{k}=\bigcup_{2 k<n} \hat{A}_{2 k} \backslash \hat{A}_{2 k+1},
$$

and therefore

$$
n-\Gamma=\left\{\bigcup_{2 k<n} A_{2 k} \backslash A_{2 k+1} \mid A_{0} \supseteq A_{1} \supseteq \cdots \supseteq A_{n}=\emptyset \& \forall k<n\left(A_{k} \in \boldsymbol{\Gamma}\right)\right\} .
$$

Suppose now that $\boldsymbol{\Gamma}$ and $\breve{\boldsymbol{\Gamma}}$ are both closed under finite unions or, equivalently, $\boldsymbol{\Gamma}$ is closed under finite unions and intersections. If $A \in \boldsymbol{\Gamma}$ and $B=\bigcup_{2 k<n} B_{2 k} \backslash B_{2 k+1} \in$ $n$ - $\boldsymbol{\Gamma}$, then $A \cap B=\bigcup_{2 k<n}\left(A \cap B_{2 k}\right) \backslash\left(A \cap B_{2 k+1}\right) \in n$ - $\boldsymbol{\Gamma}$, i.e., the pointclass $n-\boldsymbol{\Gamma}$ is closed under intersections with sets in $\Gamma$. For any fixed $n$, letting $\check{A}_{k}=\neg A_{2 n-1-k}$ for $k<2 n$, defines a bijection

$$
\left\langle A_{0}, \ldots, A_{2 n-1}\right\rangle \mapsto\left\langle\check{A}_{0}, \ldots, \check{A}_{2 n-1}\right\rangle,
$$

between the family of all decreasing $2 n$-tuples of sets in $\Gamma$ and the family of all decreasing $2 n$-tuples of sets in $\breve{\Gamma}$. Since $\bigcup_{k<n} A_{2 k} \backslash A_{2 k+1}=\bigcup_{k<n} \check{A}_{2 k} \backslash \check{A}_{2 k+1}$, it follows that $2 n-\boldsymbol{\Gamma}=2 n-\breve{\Gamma}$. Similarly, for every fixed $n$, letting $\tilde{A}_{k}=\neg A_{2 n-k}$ for $k \leq 2 n$, defines a bijection

$$
\left\langle A_{0}, \ldots, A_{2 n}, \emptyset\right\rangle \mapsto\left\langle\tilde{A}_{0}, \ldots, \tilde{A}_{2 n}, \emptyset\right\rangle,
$$

between the family of all decreasing $(2 n+2)$-tuples of sets in $\boldsymbol{\Gamma}$ with last set empty, and the family of all decreasing $(2 n+2)$-tuples of sets in $\breve{\Gamma}$ with last set empty. It is not hard to check that in this situation, letting $A_{2 n+1}=\emptyset=\tilde{A}_{2 n+1}$, then

$$
\mathbb{R} \backslash\left(\bigcup_{2 k<2 n+1} A_{2 k} \backslash A_{2 k+1}\right)=\bigcup_{2 k<2 n+1} \tilde{A}_{2 k} \backslash \tilde{A}_{2 k+1},
$$

and hence $((2 n+1)-\boldsymbol{\Gamma})^{\smile}=(2 n+1)-\breve{\Gamma}$. If $A_{0} \supseteq \cdots \supseteq A_{n+1}=\emptyset$ are in $\boldsymbol{\Gamma}$, then $\bigcup_{2 k<n+1} A_{2 k} \backslash A_{2 k+1}=A_{0} \backslash\left(\bigcup_{2 k<n} B_{2 k} \backslash B_{2 k+1}\right)$, where $B_{i}=A_{i+1}$ for $i \leq n$. Conversely, if $A$ and $B_{0} \supseteq \cdots \supseteq B_{n}=\emptyset$ are in $\Gamma$, then

$$
\begin{aligned}
A \backslash\left(\bigcup_{2 k<n} B_{2 k} \backslash B_{2 k+1}\right)=A \backslash\left(\bigcup _ { 2 k < n } ( B _ { 2 k } \cap A ) \backslash \left(B_{2 k+1}\right.\right. & \cap A)) \\
& =\bigcup_{2 k<n+1} A_{2 k} \backslash A_{2 k+1}
\end{aligned}
$$

where $A_{0}=A$ and $A_{k+1}=B_{k} \cap A$ for $k \leq n$. Therefore $(n+1)-\boldsymbol{\Gamma}=\{A \backslash B \mid A \in$ $\boldsymbol{\Gamma}, B \in n-\boldsymbol{\Gamma}\}$ and hence $(n-\boldsymbol{\Gamma})^{\smile} \subseteq(n+1)-\boldsymbol{\Gamma}$.

Let us summarize the observations above:

Lemma 11. Let $\boldsymbol{\Gamma}$ be a pointclass closed under finite unions and intersections. Then

$$
\begin{aligned}
2 n-\boldsymbol{\Gamma} & =2 n-\breve{\Gamma}, \\
(2 n+1)-\breve{\Gamma} & =((2 n+1)-\boldsymbol{\Gamma})^{\smile}, \\
(n+1)-\boldsymbol{\Gamma} & =\{A \backslash B \mid A \in \boldsymbol{\Gamma} \& B \in n-\boldsymbol{\Gamma}\} .
\end{aligned}
$$

Recall from (20) and the comments following it, that $\boldsymbol{\Lambda}_{n}$ is the $n$-th $\downarrow$-closed pointclass. 
Lemma 12. For $n \geq 1$

(a) $\Lambda_{n}=n-\Sigma_{2}^{0}$, and

(b) $\left\|n-\Sigma_{2}^{0}\right\|_{\mathrm{W}}=\left\|n-\Pi_{2}^{0}\right\|_{\mathrm{W}}=\omega_{1}^{n}+1$.

Proof. (a) The result is true for $n=1$, since $\mathrm{C}_{1}=\mathbb{R}^{\natural}$ is a complete $\boldsymbol{\Sigma}_{2}^{0}$ set, and suppose $\boldsymbol{\Lambda}_{n}=n-\boldsymbol{\Sigma}_{2}^{0}$. By construction $\boldsymbol{\Lambda}_{n+1}$ is the pointclass generated by $\mathrm{C}_{n+1}=$ $\left(\neg \mathrm{C}_{n}\right)^{\natural}$, hence by Corollary 10

$$
\begin{aligned}
\boldsymbol{\Lambda}_{n+1} & =\left\{\bigcup_{m}\left(F_{m} \cap A_{m}\right) \mid F_{m} \in \Pi_{1}^{0} \text { are pairwise disjoint and } A_{m} \leq{ }_{\mathrm{W}} \neg \mathrm{C}_{n}\right\} \\
& =\left\{\bigcup_{m}\left(F_{m} \backslash A_{m}\right) \mid F_{m} \in \Pi_{1}^{0} \text { are pairwise disjoint and } A_{m} \in n-\Sigma_{2}^{0}\right\} .
\end{aligned}
$$

Replacing each $A_{m}$ with $A_{m} \cap F_{m}$ (which can be done by Lemma 6), we may assume $A_{m} \subseteq F_{m}$. Each $A_{m}$ is of the form $\bigcup_{2 k<n} A_{2 k}^{m} \backslash A_{2 k+1}^{m}$ with $A_{i}^{m} \in \Sigma_{2}^{0}$, and again we may assume $F_{m} \supseteq A_{0}^{m} \supseteq \cdots \supseteq A_{n-1}^{m}$ for every $m \in \omega$. Since the $F_{m}$ 's are disjoint, then the $\left\{A_{i}^{m} \mid m \in \omega\right\}$ are disjoint, for any fixed $i<n$, and

$$
\bigcup_{m} A_{m}=\bigcup_{m}\left(\bigcup_{2 k<n} A_{2 k}^{m} \backslash A_{2 k+1}^{m}\right)=\bigcup_{2 k<n} B_{2 k} \backslash B_{2 k+1}
$$

where $B_{i}=\bigcup_{m} A_{i}^{m} \in \Sigma_{2}^{0}$. Therefore

$$
\bigcup_{m}\left(F_{m} \backslash A_{m}\right)=\left(\bigcup_{m} F_{m}\right) \backslash\left(\bigcup_{m} A_{m}\right)=\left(\bigcup_{m} F_{m}\right) \backslash\left(\bigcup_{2 k<n} B_{2 k} \backslash B_{2 k+1}\right)
$$

is the difference between a set in $\Sigma_{2}^{0}$ and a set in $n-\Sigma_{2}^{0}$, and therefore it is a set in $(n+1)-\Sigma_{2}^{0}$ by Lemma 11 .

Conversely, we must show that an arbitrary element of $(n+1)-\boldsymbol{\Sigma}_{2}^{0}$ is in $\boldsymbol{\Lambda}_{n+1}$. By Lemma 11 every element in $(n+1)-\Sigma_{2}^{0}$ is of the form $F \backslash A$, with $F \in \Sigma_{2}^{0}$ and $A \in n-\Sigma_{2}^{0}$. Pick $F_{m}$ closed and disjoint such that $F=\bigcup_{m} F_{m}$, and let $A_{0} \supseteq \cdots \supseteq$ $A_{n}=\emptyset$ be in $\Sigma_{2}^{0}$ and such that $A=\bigcup_{2 k<n} A_{2 k} \backslash A_{2 k+1}$. Let $B_{k}^{m}=A_{k} \cap F_{m} \in n-\Sigma_{2}^{0}$, let $B_{m}=\bigcup_{2 k<n} B_{2 k}^{m} \backslash B_{2 k+1}^{m} \in n-\Sigma_{2}^{0}$. Then $F \backslash A=\bigcup_{m}\left(F_{m} \backslash B_{m}\right) \in \Lambda_{n+1}$.

(b) follows from (a) and the observations after (20).

B.5. The stretch operation. Let $\sigma:{ }^{<\omega} \omega \rightarrow{ }^{<\omega} \omega$ be defined by $\sigma(\emptyset)=\emptyset, \sigma\left(s^{\frown} 0\right)=$ $\sigma(s)$, and $\sigma\left(s^{\frown}(n+1)\right)=\sigma(s)^{\wedge} n$. Then $\sigma$ induces a continuous map $\boldsymbol{S}: P \rightarrow \mathbb{R}$, where

$$
P=\left\{x \in \mathbb{R} \mid \exists^{\infty} n x(n) \neq 0\right\} .
$$

The real $\boldsymbol{S}(x)$ is obtained from $x$ by first eliminating all 0's and then subtracting 1 from all the surviving entries. The stretch of $A \subseteq \mathbb{R}$ is

$$
A^{\text {str }}=\boldsymbol{S}^{-1} \text { " } A \text {. }
$$

The basic properties of the stretch operation are:

(23) $\overrightarrow{0} \in\left[\boldsymbol{T}\left(A^{\text {str }}\right)\right]$, hence $A^{\text {str }}$ is non-self-dual.

(24) $A \leq_{\mathrm{W}} B \Longrightarrow A \leq_{\mathrm{W}} A^{\text {str }} \leq_{\mathrm{W}} B^{\text {str }}$ (monotonicity).

(25) $\left(A^{\mathrm{str}}\right)^{\mathrm{str}} \equiv_{\mathrm{W}} A^{\mathrm{str}}$ (idempotence).

(26) $(A+B)^{\mathrm{str}}=A^{\mathrm{str}}+B^{\mathrm{str}}$. 
(27) $\emptyset^{\text {str }}=\emptyset$ and $\mathbb{R}^{\text {str }}=P \in \boldsymbol{\Pi}_{2}^{0} \backslash \Sigma_{2}^{0}$, where $P$ is as in (22). Hence by monotonicity and idempotence

$$
\forall A \in \Delta_{2}^{0} \backslash\{\emptyset\}\left(A^{\text {str }} \in \Pi_{2}^{0} \backslash \Sigma_{2}^{0}\right) .
$$

Moreover, in [VW77] it is shown that

(28) (Van Wesep) If $A$ is self-dual and $\forall B<_{\mathrm{W}} A\left(B^{\text {str }}<_{\mathrm{W}} A\right)$, then $A^{\text {str }} \equiv_{\mathrm{W}} A+\emptyset$.

(29) If $\|A\|_{\mathrm{W}}=\omega_{1} \cdot\|A\|_{\mathrm{W}}$ is of uncountable cofinality, then $A^{\mathrm{str}} \equiv_{\mathrm{W}} A$.

Lemma 13. If $B \leq_{\mathrm{W}} A$ and $G \in \Pi_{2}^{0}$ then $B \cap G \leq_{\mathrm{W}} A^{\text {str }}$.

Proof. Let $G=\bigcap_{n} U_{n}$ with $U_{n}$ open and fix a winning strategy $\tau$ for II in $G_{\mathrm{W}}(B, A)$. Keeping in mind that $\boldsymbol{N}_{s}$ is the basic open neighborhood determined by $s$, and that $\tau$ has the option of passing, let II play in $G_{\mathrm{L}}\left(B \cap G, A^{\text {str }}\right)$ as follows:

II plays 0 as long as $\mathbf{I}$ does not reach a position $p_{0}$ such that $\boldsymbol{N}_{p_{0}} \subseteq U_{0}$ and such that $\tau$ has actually produced an output (and did not simply pass all the time). If such a position $p_{0}$ is reached, let $q_{0} \subseteq p_{0}$ be least such that $\tau$ applied to $q_{0}$ yields a sequence of length 1 , and let II play $\tau\left(q_{0}\right)+1$.

Then II plays 0 as long as $\mathbf{I}$ does not reach a position $p_{1} \supseteq p_{0}$ such that $\boldsymbol{N}_{p_{1}} \subseteq U_{1}$ and such that there is a least $q_{1} \subseteq p_{1}$ such that $\tau$ applied to $q_{1}$ yields a sequence of length 2. If such a position $p_{1}$ is reached, then II plays $\tau\left(q_{1}\right)+1$.

And so on.

It is easy to check that this is a winning strategy.

Say that a pointclass $\boldsymbol{\Gamma}$ is closed under stretches if $A \in \boldsymbol{\Gamma} \Longrightarrow A^{\text {str }} \in \boldsymbol{\Gamma}$. Thus the non-self-dual pointclasses closed under stretches are of the form $\left\{B \mid B \leq_{\mathrm{W}} A^{\text {str }}\right\}$ for some $A$.

Lemma 14. The first $\omega$ pointclasses closed under stretches are $\{\emptyset\}$ and the $n-\Pi_{2}^{0}$, with $n \geq 1$.

Proof. That $\{\emptyset\}$ and $\Pi_{2}^{0}$ are the 0-th and 1-st pointclass closed under stretches follows from (25) and (27). Assume inductively that $n-\Pi_{2}^{0}$ is the $n$-th such pointclass. The next pointclass closed under stretches is $\left\{X \mid X \leq_{\mathrm{W}} A^{\text {str }}\right\}$ where $A$ is complete for $\left(n-\Pi_{2}^{0}\right)^{\smile}$. By Lemmas 11 and 13,

$$
(n+1)-\Pi_{2}^{0}=\left\{B \cap G \mid B \leq_{\mathrm{W}} A \& G \in \Pi_{2}^{0}\right\} \subseteq\left\{X \mid X \leq_{\mathrm{W}} A^{\text {str }}\right\} .
$$

To show the other inclusion, it is enough to show that $A^{\text {str }} \in(n+1)-\Pi_{2}^{0}$. Notice that the definition of $n-\Pi_{2}^{0}$ makes sense in every topological space; in particular, since the set $P$ of $(22)$ is $\boldsymbol{\Pi}_{2}^{0}$, if $X \subseteq P$ is $\left(n-\Pi_{2}^{0}\right)^{\smile}$ in the space $P$ then there is a $\tilde{X} \in\left(n-\Pi_{2}^{0}\right)^{\smile}$ such that $X=\tilde{X} \cap P$, and therefore $X \in(n+1)-\Pi_{2}^{0}$. As the function $\boldsymbol{S}: P \rightarrow \mathbb{R}$ used to define the stretch operation is continuous, then $A^{\text {str }}=S^{-1}$ " $A$ is $\left(n-\boldsymbol{\Pi}_{2}^{0}\right)^{\smile}$ in the space $P$, hence $A^{\text {str }} \in(n+1)-\Pi_{2}^{0}$. 
In analogy with (19), let

$$
\mathrm{D}_{0}=\emptyset, \quad \mathrm{D}_{n+1}=\left(\neg \mathrm{D}_{n}\right)^{\mathrm{str}}, \quad \mathrm{D}_{\omega}=\bigoplus_{n} \mathrm{D}_{n} .
$$

Then by Lemma 14,

(30) For $0<n<\omega$, the set $\mathrm{D}_{n}$ is complete for $n-\Pi_{2}^{0}$, and therefore $\left\|\mathrm{D}_{n}\right\|_{\mathrm{W}}=\omega_{1}^{n}$.

The first $\omega$ non-trivial pointclasses closed under stretches are the $n-\Pi_{2}^{0}$, and they are all non-self-dual. Since the increasing union of pointclasses closed under stretches is closed under stretches, then

$$
<\omega-\Pi_{2}^{0}=\bigcup_{n} n-\Pi_{2}^{0}=\left\{B \mid B<_{\mathrm{W}} \mathrm{D}_{\omega}\right\}
$$

is the $\omega+1$-st pointclass closed under stretches.

By properties (24)-(30) above, a complete analysis of the stretch operation on degrees can be given.

(31) Let $\boldsymbol{\Gamma}$ be closed under stretches.

If $\boldsymbol{\Gamma}$ is self-dual, then $\|\boldsymbol{\Gamma}\|_{\mathrm{W}}=\omega_{1}^{\omega} \cdot \gamma$, with $\gamma>0$.

If $\boldsymbol{\Gamma}$ is non-self-dual, then either

$\|\boldsymbol{\Gamma}\|_{\mathrm{W}}=\omega_{1}^{\omega} \cdot \gamma+1$ with $\gamma$ limit and $\operatorname{cof}(\gamma)>\omega$, or else

$\|\boldsymbol{\Gamma}\|_{\mathrm{W}}=\omega_{1}^{\omega} \cdot \gamma+2$ with $\gamma$ successor or limit and $\operatorname{cof}(\gamma)=\omega$, or else

$\|\boldsymbol{\Gamma}\|_{\mathrm{W}}=\omega_{1}^{\omega} \cdot \gamma+\omega_{1}^{n}+1$ with $\gamma \geq 0$ and $n>0$.

Conversely:

Suppose $\boldsymbol{\Gamma}$ is self-dual. If $\|\boldsymbol{\Gamma}\|_{\mathrm{W}}=\omega_{1}^{\omega} \cdot \gamma$ with $\gamma>0$, then $\boldsymbol{\Gamma}$ is closed under stretches.

Suppose $\boldsymbol{\Gamma}$ is non-self-dual. If either

$\|\boldsymbol{\Gamma}\|_{\mathrm{W}}=\omega_{1}^{\omega} \cdot \gamma+\omega_{1}^{n}+1$ with $\gamma \geq 0$ and $n>0$, or else

$\|\boldsymbol{\Gamma}\|_{\mathrm{W}}=\omega_{1}^{\omega} \cdot \gamma+2$ with $\gamma>0$ successor or limit of countable cofinality, then exactly one among $\boldsymbol{\Gamma}$ and $\breve{\Gamma}$ is closed under stretches,

If $\|\boldsymbol{\Gamma}\|_{\mathrm{W}}=\omega_{1}^{\omega} \cdot \gamma+1$ with $\operatorname{cof}(\gamma)>\omega$, then both $\boldsymbol{\Gamma}$ and $\breve{\boldsymbol{\Gamma}}$ are closed under stretches.

Note that $\mathrm{D}_{n} \equiv_{\mathrm{W}} \mathrm{C}_{n}$ if $n$ is even and $\mathrm{D}_{n} \equiv_{\mathrm{W}} \neg \mathrm{C}_{n}$ if $n$ is odd. Here is how the stretch and $\downarrow$ operations act on the pointclasses $n-\Sigma_{2}^{0}$ and $n-\Pi_{2}^{0}$ :

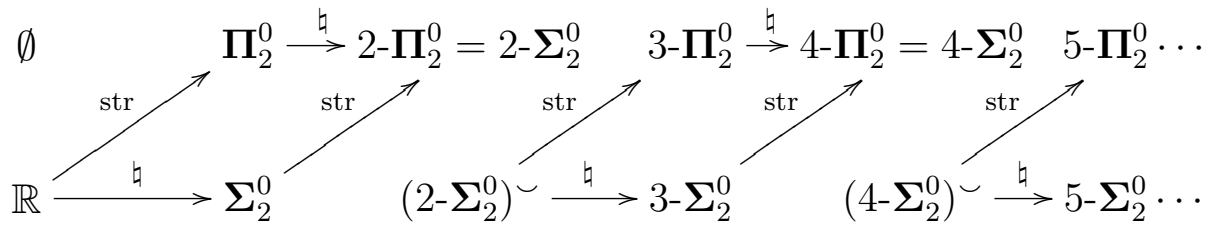

The pointclass immediately above these is $\left\langle\omega-\Sigma_{2}^{0}=\bigcup_{n} n-\Sigma_{2}^{0}=\bigcup_{n} n-\Pi_{2}^{0}\right.$, and it is closed both under the stretch and $\downarrow$ operations. After this stage, the two operations are not entwined any more, since the ranks of $\downarrow$-closed pointclasses grow as powers of $\omega_{1}$, while the ranks of the stretch-closed ones exhibit a "periodicity" of period $\omega_{1}^{\omega}$ : 
in fact the next $\downarrow$-closed pointclass above $<\omega$ - $\Sigma_{2}^{0}$ has rank $\omega_{1}^{\omega+1}+1$, while the next stretch-closed pointclass has rank $\omega_{1}^{\omega}+\omega_{1}+1$.

We now prove a technical result which will be useful in $\S 6$.

A partial function $f$ from the reals to the reals, is a -function iff $\operatorname{dom}(f)=$ $\bigcup_{n} C_{n} \subseteq \mathbb{R}$ and

(i) $C_{n}$ is closed non-empty,

(ii) $C_{n} \cap C_{m}=\emptyset$, if $n \neq m$,

(iii) $f \uparrow C_{n}: C_{n} \rightarrow \mathbb{R}$ is Lipschitz.

As the $\downarrow$-functions will play an important role in later sections, let us fix-once and for all - a parametrization of these functions via reals. A $\downarrow$-function is coded by a sequence

$$
\left\langle\left(T_{n}, \Phi_{n}\right) \mid n \in \omega\right\rangle
$$

where each $T_{n}$ is a pruned tree on $\omega$, each $\Phi_{n}: T_{n} \rightarrow<\omega \omega$ is Lipschitz, and $\left[T_{n}\right] \cap$ $\left[T_{m}\right]=\emptyset$, for $n \neq m$. In fact we may even assume that the stronger condition that $T_{n} \cap T_{m}$ is finite, for $n \neq m$, since by repeated applications of the next lemma, we can inductively construct almost disjoint $T_{n}^{\prime}$ 's such that $\bigcup_{n}\left[T_{n}\right]=\bigcup_{n}\left[T_{n}^{\prime}\right]$.

Lemma 15. Suppose $S, U$ are pruned trees on $\omega$ such that $[S] \cap[T]=\emptyset$ but $\mid S \cap U=\omega$. Then there are pruned trees $U_{n} \subseteq U$ such that $[U]=\bigcup_{n \in \omega}\left[U_{n}\right]$ and $S \cap U_{n}$ and $U_{n} \cap U_{m}(n \neq m)$ are all finite.

Proof. Since the closed sets defined by $S$ and $U$ are disjoint, the tree $S \cap U$ is infinite and well-founded. Let $\left\langle t_{n} \mid n \geq 1\right\rangle$ be an enumeration of the terminal nodes on $S \cap U$. Then $D=\left\{x \in \mathbb{R} \mid \exists n \geq 1\left(x \supset t_{n}\right)\right\}$ is clopen, so we can set $U_{0}$ to be the unique pruned tree such that $\left[U_{0}\right]=[U] \backslash D$ and

$$
U_{n}=\left\{u \in U \mid u \supseteq t_{n} \vee t_{n} \subseteq u\right\} \quad(n>0) .
$$

Recall from (8) that $\boldsymbol{H}$ is the set of all sequences of pruned trees and Lipschitz functions defined on them. Then

$$
\boldsymbol{F}=\left\{\left\langle\left(T_{n}, \Phi_{n}\right) \mid n \in \omega\right\rangle \in \boldsymbol{H} \mid \forall n, m\left(n \neq m \Longrightarrow\left|T_{n} \cap T_{m}\right|<\omega\right)\right\}
$$

is the set of codes for $\downarrow$-functions, since every element of $\boldsymbol{F}$ yields a $\downarrow$-function $\bigcup_{n} f_{n}: \bigcup_{n}\left[T_{n}\right] \rightarrow \mathbb{R}$, where $f_{n}:\left[T_{n}\right] \rightarrow \mathbb{R}$ is the Lipschitz map induced by $\Phi_{n}$. Arguing as for $\boldsymbol{H}$, the set $\boldsymbol{F}$ can be identified with a $\mathbf{G}_{\delta}$ subset $\mathcal{F}$ of ${ }^{\omega} 2$, and hence of $\mathbb{R}$ : the condition that the $T$ 's be almost disjoint can be written as

$$
\forall n, m \exists K \forall k\left(n \neq m \& \boldsymbol{s}_{k} \in T_{n} \cap T_{m} \Longrightarrow k \leq K\right)
$$

which is easily seen to be $\Pi_{2}^{0}$. The bijection

$$
\mathcal{F} \rightarrow \boldsymbol{F}, \quad x \mapsto\left\langle\left(T_{x, n}, \Phi_{x, n}\right) \mid n \in \omega\right\rangle,
$$

is the coding of 4 -functions via elements of $\mathcal{F}$, and we denote its inverse function with

$$
j: F \rightarrow \mathcal{F}
$$


Letting $\boldsymbol{f}_{x, n}:\left[T_{x, n}\right] \rightarrow \mathbb{R}$ be the Lipschitz function induced by $\Phi_{x, n}$, then

$$
\boldsymbol{f}_{x}=\bigcup_{n} \boldsymbol{f}_{x, n}: \bigcup_{n}\left[T_{x, n}\right] \rightarrow \mathbb{R}
$$

is the -function coded by $x \in \mathcal{F}$.

Lemma 16. Suppose $\|\boldsymbol{\Gamma}\|_{\mathrm{W}}=\omega_{1}^{\xi}+1$ with $\xi$ a successor ordinal, or a limit ordinal of uncountable cofinality. Then there is a set $A \subseteq{ }^{\omega} 2$ such that $\|A\|_{\mathrm{W}}=\omega_{1}^{\xi}$ and such that either $\boldsymbol{\Gamma}$ or $\breve{\boldsymbol{\Gamma}}$ is of the form

$$
\left\{\boldsymbol{f}_{x}^{-1} \text { “ } A \mid x \in \mathcal{F}\right\} .
$$

Proof. $\boldsymbol{\Gamma}$ and $\breve{\boldsymbol{\Gamma}}$ form a non-self-dual pair of pointclasses, and by (31) at least one among them is closed under stretches, say $\Gamma$ is such. Let $F: \mathbb{R} \rightarrow{ }^{\omega_{2}}$ be the Lipschitz map defined by

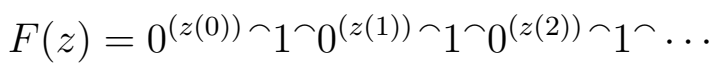

Trivially, $F$ witnesses $B \leq_{\mathrm{W}} F$ " $B$, for any set $B$. Moreover:

Claim 17. $F^{\text {" }} B \leq_{\mathrm{W}} B^{\mathrm{str}}$.

Proof of Claim. Consider the following strategy for II in $G_{\mathrm{L}}\left(F^{\prime} B, B^{\text {str }}\right)$ :

If at some round $\mathbf{I}$ plays an integer different from 0 and 1 , then II plays 0 from this point on. So suppose that $\mathbf{I}$ has been playing elements of $\{0,1\}$ : if $\mathbf{I}$ plays 0 , then II answers 0 , if $\mathbf{I}$ plays 1 , then II answers $n+1$, where $n$ is the number of rounds since $\mathbf{I}$ last played 1 (or the total number of rounds since the beginning of the game, if this is the first time $\mathbf{I}$ plays 1 ).

Let $x$ and $y$ be the reals played by $\mathbf{I}$ and $\mathbf{I I}$, respectively: if either $x \notin{ }^{\omega} 2$ or $\forall^{\infty} n x(n)=0$, then $y$ is of the form $s-\overrightarrow{0}$. Otherwise $x$ is of the form

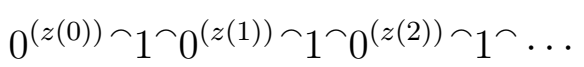

and $y=z+1$. Therefore $F^{\text {" }} B \leq_{\mathrm{W}} B^{\text {str }}$.

Thus if $B$ is $\boldsymbol{\Gamma}$-complete and $A=F$ " $B$, then $A \subseteq{ }^{\omega} 2$ and $A \equiv_{\mathrm{W}} B$, so fix such an $A$. We must show that $\boldsymbol{\Gamma}$ is the collection of sets in (36).

Let $X$ be a set in $\Gamma$. By Theorem 2 there is a Lipschitz $f: \mathbb{R} \rightarrow \mathbb{R}$ such that $f^{-1}$ " $A=X$. Since $f$ is a $\downarrow$-function (let $C_{n}=\boldsymbol{N}_{\langle n\rangle}$ and $f_{n}=f\left\lceil C_{n}\right.$ ) then $X$ is in the set in (36). Conversely, we must show that every set in (36) is in $\Gamma$. Let $f_{n}: C_{n} \rightarrow \mathbb{R}$ be Lipschitz, with $C_{n} \subseteq \mathbb{R}$ closed and pairwise disjoint, and let $f=\bigcup_{n} f_{n}$. If $C_{n}=\emptyset$ for all $n$, then $f^{-1} " A=\emptyset \in \Gamma$, so we may assume that $Y=\left\{n \in \omega \mid C_{n} \neq \emptyset\right\}$ is non-empty. For each $n \in Y$ let $r_{n}: \mathbb{R} \rightarrow C_{n}$ be a retraction of $\mathbb{R}$ onto $C_{n}$. Then $f_{n} \circ r_{n}: \mathbb{R} \rightarrow \mathbb{R}$ is continuous (in fact: Lipschitz) and

$$
f^{-1 "} A=\bigcup_{n \in Y} C_{n} \cap\left(f_{n} \circ r_{n}\right)^{-1 "} A
$$

By (21) $\boldsymbol{\Gamma}$ is $\downarrow$-closed hence $f^{-1}$ " $A \in \boldsymbol{\Gamma}$ by Lemma 9 . 


\section{Cardinality of pointclasses}

In this section we will characterize the cardinality pointclasses, i.e., the non-trivial pointclasses $\boldsymbol{\Gamma}$ such that

$$
\forall \Gamma^{\prime} \subset \Gamma\left(\left|\Gamma^{\prime}\right|<|\Gamma|\right) .
$$

Lemma 18. Suppose $\boldsymbol{\Gamma}$ is either principal or such that $\operatorname{cof}\left(\|\boldsymbol{\Gamma}\|_{\mathrm{W}}\right)>\omega$. Then $\left|{ }^{\omega} \boldsymbol{\Gamma}\right|=|\boldsymbol{\Gamma}|$.

Proof. Given $A_{n} \in \boldsymbol{\Gamma}$, we will show that $\bigoplus_{n} A_{n} \leq_{\mathrm{W}} A$, for some $A \in \boldsymbol{\Gamma}$. Since the map $\left\langle A_{n} \mid n \in \omega\right\rangle \mapsto \bigoplus_{n} A_{n}$ is injective, this will prove the result. If $A$ is $\boldsymbol{\Gamma}$ complete, then $\bigoplus_{n} A_{n} \leq_{\mathrm{W}} A$ and we are done. Suppose instead $\boldsymbol{\Gamma}$ is non-principal and $\operatorname{cof}\left(\|\boldsymbol{\Gamma}\|_{\mathrm{W}}\right)>\omega$, and let $A_{n} \in \boldsymbol{\Gamma}$. By case assumption there is an $\alpha$ such that $\sup \left\{\left\|A_{n}\right\|_{\mathrm{W}} \mid n \in \omega\right\} \leq \alpha<\|\boldsymbol{\Gamma}\|_{\mathrm{W}}$, and let $A$ be such that $\alpha=\|A\|_{\mathrm{W}}$. Then $A \in \boldsymbol{\Gamma}$ and $\bigoplus_{n} A_{n} \leq_{\mathrm{W}} A$.

The following result uses the non-triviality of pointclasses in an essential way.

Corollary 19. If $\boldsymbol{\Gamma}$ is any non-trivial pointclass, then $\left.\right|^{<\omega} \boldsymbol{\Gamma}|=| \boldsymbol{\Gamma} \mid$. In particular, $|\boldsymbol{\Gamma} \cup \breve{\boldsymbol{\Gamma}}|=|\boldsymbol{\Gamma}|$, and hence $\left.\right|^{\omega}(\boldsymbol{\Gamma} \cup \breve{\boldsymbol{\Gamma}})|=|{ }^{\omega} \boldsymbol{\Gamma} \mid$.

Proof. Since $\mathbb{R} \rightarrow \boldsymbol{\Gamma}$ and $\boldsymbol{\Gamma}$ is infinite, by $\mathrm{DC}(\mathbb{R})$ there is a sequence $\emptyset=X_{0}, X_{1}, \ldots$ of distinct elements of $\boldsymbol{\Gamma}$, hence $|\boldsymbol{\Gamma} \backslash\{\emptyset\}|=|\boldsymbol{\Gamma}|$. Thus it is enough to show that $\left.\right|^{<\omega}(\boldsymbol{\Gamma} \backslash$ $\{\emptyset\})|\leq| \boldsymbol{\Gamma} \mid$. As the map ${ }^{<\omega}(\boldsymbol{\Gamma} \backslash\{\emptyset\}) \rightarrow{ }^{\omega} \boldsymbol{\Gamma},\left\langle A_{0}, \ldots, A_{n}\right\rangle \mapsto\left\langle A_{0}, \ldots, A_{n}, \emptyset, \emptyset, \ldots\right\rangle$ is injective, then we are done by Lemma 18 if $\boldsymbol{\Gamma}$ is principal or $\operatorname{cof}\left(\|\boldsymbol{\Gamma}\|_{\mathrm{W}}\right)>\omega$. Suppose instead that $\boldsymbol{\Gamma}=\left\{B \mid B<_{\mathrm{W}} A\right\}$ with $A$ self-dual and limit. Given $B_{0}, \ldots, B_{n} \in \Gamma \backslash\{\emptyset\}$, consider $\bigoplus_{i} B_{i}$, where we set $B_{m}=\emptyset$ for all $m>n$; then $\bigoplus_{i} B_{i}<_{\mathrm{W}} A$. Since the map $\left\langle B_{0}, \ldots, B_{n}\right\rangle \mapsto \bigoplus_{i} B_{i}$ is injective we are done.

Lemma 20. Let $A \neq \emptyset, \mathbb{R}$ be non-self-dual, and let $\Gamma$ be the pointclass generated by $A$. Then the pointclass generated by $A \oplus \neg A$ has cardinality $|\boldsymbol{\Gamma}|$.

Proof. If $X \leq_{\mathrm{W}} A \oplus \neg A$ then either $X \in \boldsymbol{\Gamma}$, or $X \in \breve{\Gamma}$, or else $X \equiv_{\mathrm{W}} A \oplus \neg A$, and thus by Corollary 19 it is enough to show that $\left|[A \oplus \neg A]_{\mathrm{W}}\right| \leq|\boldsymbol{\Gamma}|$. If $X \in[A \oplus \neg A]_{\mathrm{W}}$ then $X$ can be recovered from the tree $\boldsymbol{T}(X)$ and the sequence $\mathcal{S}(X)=\left\langle X_{\lfloor s\rfloor}\right| s \in$ $\partial \boldsymbol{T}(X)\rangle$, since

$$
X=\bigcup\left\{s^{\wedge} X_{\lfloor s\rfloor} \mid s \in \partial \boldsymbol{T}(X)\right\} .
$$

Therefore the map $X \mapsto(\boldsymbol{T}(X), \mathcal{S}(X))$ is injective and witnesses that

$$
\begin{aligned}
\left|[A \oplus \neg A]_{\mathrm{W}}\right| & \leq \mid \text { Trees } \times{ }^{\omega}(\boldsymbol{\Gamma} \cup \breve{\boldsymbol{\Gamma}}) \mid & & \\
& \leq|\mathbb{R}| \cdot\left|{ }^{\omega} \boldsymbol{\Gamma}\right| & & \text { (by Corollary 19) } \\
& \leq|\boldsymbol{\Gamma}| . & & \text { (by Lemma 18) }
\end{aligned}
$$

Lemma 21. Let $A$ be self-dual, and let $\boldsymbol{\Gamma}$ be the pointclass generated by $A$. Then the pointclass generated by $A+A$ has cardinality $|\boldsymbol{\Gamma}|$. 
Proof. Let $\Gamma^{\prime}=\left\{X \mid X \leq_{\mathrm{W}} A+A\right\}$. Since $\Gamma^{\prime}=\boldsymbol{\Gamma} \cup\left\{X \in \Gamma^{\prime} \mid A<_{\mathrm{W}} X\right\}$, then by Corollary 19, it is enough to prove that

$$
\left|\left\{X \in \Gamma^{\prime} \mid A<_{\mathrm{W}} X\right\}\right| \leq|\Gamma| .
$$

For $X \in \Gamma^{\prime}$ such that $A<_{\mathrm{W}} X$ we can construct the tree $T_{X}=\boldsymbol{T}\left(X ;\|A\|_{\mathrm{W}}+1\right)$ and the sequence

$$
\mathcal{S}_{X}=\left\langle X_{\lfloor s\rfloor} \mid s \in \partial T_{X}\right\rangle
$$

of sets in $\boldsymbol{\Gamma}$. By Lemma 3 the tree $T$ is pruned so that $\boldsymbol{r}_{T_{X}}: \mathbb{R} \rightarrow\left[T_{X}\right]$, the canonical Lipschitz retraction onto $\left[T_{X}\right]$, is defined. The set $C_{X}=\boldsymbol{r}_{T_{X}}^{-1}$ "([$\left.\left[T_{X}\right] \cap X\right)$ is Wadge reducible to $A$ via $z \mapsto f\left(\boldsymbol{r}_{T_{X}}(z)\right)-1$, where $f$ is the function witnessing $X \leq_{\mathrm{W}} A+A$, and therefore $C_{X} \in \boldsymbol{\Gamma}$. The set $X$ can be reconstructed from $T_{X}, \mathcal{S}_{X}$, and $C_{X}$, as

$$
X=\left(C_{X} \cap\left[T_{X}\right]\right) \cup \bigcup\left\{s^{\wedge} X_{\lfloor s\rfloor} \mid s \in \partial T_{X}\right\},
$$

hence the map $X \mapsto\left(T_{X}, \mathcal{S}_{X}, C_{X}\right)$ is injective. Therefore

$$
\left|\left\{X \in \Gamma^{\prime} \mid A<_{\mathrm{W}} X\right\}\right| \leq \mid \text { Trees } \times{ }^{\omega} \boldsymbol{\Gamma} \times \boldsymbol{\Gamma}|\leq| \mathbb{R}|\cdot| \boldsymbol{\Gamma}|=| \boldsymbol{\Gamma} \mid .
$$

Corollary 19 says that, as far as cardinality is concerned, we might as well restrict our attention to self-dual pointclasses.

Definition 22. A self-dual pointclass $\Delta$ is a cardinality level iff

$$
\forall \boldsymbol{\Delta}^{\prime} \subset \boldsymbol{\Delta}\left(\boldsymbol{\Delta}^{\prime} \text { self-dual } \Longrightarrow\left|\boldsymbol{\Delta}^{\prime}\right|<|\boldsymbol{\Delta}|\right) .
$$

Since the self-dual pointclasses are exactly the $\mathscr{P}^{(\alpha)}(\mathbb{R})$ 's, the definition can be rephrased as follows:

$$
\mathscr{P}^{(\alpha)}(\mathbb{R}) \text { is a cardinality level } \Longleftrightarrow \forall \beta<\alpha\left(\left|\mathscr{P}^{(\beta)}(\mathbb{R})\right|<\left|\mathscr{P}^{(\alpha)}(\mathbb{R})\right|\right) .
$$

If $\boldsymbol{\Gamma}$ is a cardinality pointclass then either it is self-dual - and hence it is a cardinality level — or else it is non-self-dual and hence $\boldsymbol{\Gamma} \cup \breve{\Gamma}$ is a cardinality level. Conversely, if $\boldsymbol{\Gamma}$ is a cardinality level, then either it is a cardinality pointclass, or else it is of the form $\boldsymbol{\Gamma}=\boldsymbol{\Lambda} \cup \breve{\Lambda}$ with $\boldsymbol{\Lambda}$ a non-self-dual cardinality pointclass. Therefore our original goal of determining all cardinality pointclasses amounts to characterizing all $\alpha$ 's such that $\mathscr{P}^{(\alpha)}(\mathbb{R})$ is a cardinality level.

Lemma 23. If $\alpha$ is limit and $\operatorname{cof}(\alpha)=\omega$, then

$$
\left|\mathscr{P}^{(\alpha+1)}(\mathbb{R})\right|=\left.\right|^{\omega}\left(\mathscr{P}^{(\alpha)}(\mathbb{R})\right) \mid \text {. }
$$

Moreover, if each $\alpha_{n}>1$ and $\sup _{n} \alpha_{n}=\alpha$, then

$$
\left|\mathscr{P}^{(\alpha+1)}(\mathbb{R})\right|=\left|\left\{\left\langle X_{n} \mid n \in \omega\right\rangle \mid \forall n\left\|X_{n}\right\|_{\mathrm{W}}<\alpha_{n}\right\}\right| .
$$

Proof. Let $A$ be such that $\|A\|_{\mathrm{W}}=\alpha$ and, for the ease of notation, let $\boldsymbol{\Gamma}=\mathscr{P}^{(\alpha)}(\mathbb{R})$. Then $A$ is self-dual and $\mathscr{P}^{(\alpha+1)}(\mathbb{R})=[A]_{\mathrm{W}} \cup \boldsymbol{\Gamma}$, so it is enough to prove that $\left|[A]_{\mathrm{W}}\right|=\left|{ }^{\omega} \boldsymbol{\Gamma}\right|$. If $B \equiv_{\mathrm{W}} A$, then $\boldsymbol{T}(B)$ is well-founded and the map

$$
B \mapsto\left(\boldsymbol{T}(B),\left\langle B_{\lfloor s\rfloor} \mid s \in \partial \boldsymbol{T}(B)\right\rangle\right)
$$


witnesses that $\left|[A]_{\mathrm{W}}\right| \leq \mid$ Trees $\times{ }^{\omega} \boldsymbol{\Gamma}|=|{ }^{\omega} \boldsymbol{\Gamma} \mid$. Conversely, the map ${ }^{\omega} \boldsymbol{\Gamma} \longmapsto[A]_{\mathrm{W}}$

$$
\left\langle X_{n} \mid n \in \omega\right\rangle \mapsto \bigoplus_{n} B_{n}
$$

where $B_{2 n}=X_{n}$ and $B_{2 n+1}=C_{n}$, where $\left\langle C_{n} \mid n<\omega\right\rangle$ is a fixed sequence of sets in $\boldsymbol{\Gamma}$ such that $\lim _{n}\left\|C_{n}\right\|_{\mathrm{W}}=\alpha$, witnesses that $\left|{ }^{\omega} \boldsymbol{\Gamma}\right| \leq\left|[A]_{\mathrm{W}}\right|$.

The second part follows immediately from the first.

Clearly, if $\boldsymbol{\Gamma}_{n} \subset \boldsymbol{\Gamma}_{n+1}$ and $\left|\boldsymbol{\Gamma}_{n}\right|<\left|\boldsymbol{\Gamma}_{n+1}\right|$ for all $n$, then $\bigcup_{n} \boldsymbol{\Gamma}_{n}$ is a cardinality level. If $\alpha=\left\|\bigcup_{n} \boldsymbol{\Gamma}_{n}\right\|_{\mathrm{W}}=\sup _{n}\left\|\boldsymbol{\Gamma}_{n}\right\|_{\mathrm{W}}$, then $\left|\bigcup_{n} \boldsymbol{\Gamma}_{n}\right| \leq\left|\mathscr{P}^{(\alpha+1)}(\mathbb{R})\right|=\left|\omega\left(\mathscr{P}^{(\alpha)}(\mathbb{R})\right)\right|$. We will show in section 5 that the inequality is strict, i.e., that both $\mathscr{P}^{(\alpha)}(\mathbb{R})$ and $\mathscr{P}^{(\alpha+1)}(\mathbb{R})$ are cardinality levels.

By Lemma 18 and-if $\mathscr{P}^{(\alpha+1)}(\mathbb{R})$ consists of two non-self-dual pointclasses-by Corollary 19, we have that for $\alpha \geq 2$,

$$
\left|\omega\left(\mathscr{P}^{(\alpha+1)}(\mathbb{R})\right)\right|=\left|\mathscr{P}^{(\alpha+1)}(\mathbb{R})\right| .
$$

Using (14) and Lemma 21 if $\mathscr{P}^{(\alpha+1)}(\mathbb{R})$ is of the form $\left\{X \mid X \leq_{\mathrm{W}} A\right\}$ for a self-dual $A$; and otherwise using Lemma 20 to work with $\mathscr{P}^{(\alpha+2)}(\mathbb{R})$ instead of $\mathscr{P}^{(\alpha+1)}(\mathbb{R})$, we have that for $\alpha \geq 2$,

$$
\left|\mathscr{P}^{(\alpha+1)}(\mathbb{R})\right|=\left|\mathscr{P}^{((\alpha+1) \cdot 2)}(\mathbb{R})\right| .
$$

It is easy to prove by induction on $1<\gamma<\omega_{1}$ that $\left|\mathscr{P}^{(\alpha+1)}(\mathbb{R})\right|=\left|\mathscr{P}^{(\alpha \cdot \gamma)}(\mathbb{R})\right|$ : if $\gamma$ is a successor ordinal, we appeal to (38); otherwise, if $\gamma$ is limit let $0<\gamma_{0}<\gamma_{1}<\ldots$ be a sequence of ordinals converging to $\gamma$, and by inductive hypothesis (and by the Axiom of Countable Choice, $\left.\mathrm{AC}_{\omega}\right)$ fix bijections $\mathscr{P}^{\left(\alpha \cdot \gamma_{n}\right)}(\mathbb{R}) \rightarrow \mathscr{P}^{(\alpha+1)}(\mathbb{R})$ so that we can define an injection from $\bigcup_{n} \mathscr{P}^{\left(\alpha \cdot \gamma_{n}\right)}(\mathbb{R})=\mathscr{P}^{(\alpha \cdot \gamma)}(\mathbb{R})$ to ${ }^{\omega} \mathscr{P}^{(\alpha+1)}(\mathbb{R})$ : then $\left|\mathscr{P}^{(\alpha \cdot \gamma)}(\mathbb{R})\right| \leq\left|\mathscr{P}^{(\alpha+1)}(\mathbb{R})\right|$ by $(37)$, and since the reverse inequality is trivially true, equality holds. The next result shows that this process can be pushed to its natural limit (and that the appeal to $\mathrm{AC}_{\omega}$ is unnecessary).

Theorem 24. If $\alpha \geq 2$ then $\left|\mathscr{P}^{(\alpha+1)}(\mathbb{R})\right|=\left|\mathscr{P}^{\left(\alpha \cdot \omega_{1}\right)}(\mathbb{R})\right|$.

Proof. Let $A$ be a set of rank $\alpha$ and suppose first that $A$ is self-dual.

To each $X \in \mathscr{P}^{\left(\alpha \cdot \omega_{1}\right)}(\mathbb{R})$ we will associate a pre-well-order $W_{X}$ of $<\omega_{\omega} \omega$ and a sequence $\mathcal{C}_{X}=\left\langle C_{X, s} \mid s \in{ }^{<\omega} \omega\right\rangle$ of sets in $\mathscr{P}^{(\alpha+1)}(\mathbb{R})$ such that the map $X \mapsto$ $\left(W_{X}, \mathrm{C}_{X}\right)$ is injective and hence

$$
\begin{aligned}
\left|\mathscr{P}^{\left(\alpha \cdot \omega_{1}\right)}(\mathbb{R})\right| & \leq\left|\mathrm{P} \operatorname{wos} \times{ }^{\omega}\left(\mathscr{P}^{(\alpha+1)}(\mathbb{R})\right)\right| \\
& =|\mathbb{R}| \cdot\left|\mathscr{P}^{(\alpha+1)}(\mathbb{R})\right| \\
& =\left|\mathscr{P}^{(\alpha+1)}(\mathbb{R})\right| .
\end{aligned}
$$

Fix $X$ of Wadge rank $<\alpha \cdot \omega_{1}$. For each $1 \leq \xi<\omega_{1}$ let

$$
T_{\xi}=\boldsymbol{T}(X ; \alpha \cdot \xi+1) .
$$

Then $\xi<\eta \Longrightarrow T_{\eta} \subseteq T_{\xi}$, and each $T_{\xi}$ is a (possibly empty) tree. By (11) and (12) it is easy to prove by induction on $0<\xi<\omega_{1}$ that there is a self-dual set of rank $\alpha \cdot \xi$, hence $T_{\xi}$ is pruned by Lemma 3. Moreover, for all $X \in \mathscr{P}^{\left(\alpha \cdot \omega_{1}\right)}(\mathbb{R})$ there is a 
least $\xi_{X}<\omega_{1}$ such that $\forall \eta \geq \xi_{X}\left(T_{\eta}=\emptyset\right)$. The sequence $\left\langle T_{\eta} \mid 1 \leq \eta \leq \xi_{X}\right\rangle$ induces a norm $\varphi=\varphi_{X}:<\omega \omega \rightarrow \xi_{X}+1$,

$$
\varphi(s)=\text { the least } \eta \text { such that } s \notin T_{\eta} \text {. }
$$

If $\varphi(s)=\eta+1$, then $s \in T_{\eta} \backslash T_{\eta+1}$, and hence $\alpha \cdot \eta+\alpha+1>\left\|X_{\lfloor s\rfloor}\right\|_{\mathrm{W}} \geq \alpha \cdot \eta+1$. If $\varphi(s)=\lambda$ is a limit ordinal, then $s \in\left(\bigcap_{\eta<\lambda} T_{\eta}\right) \backslash T_{\lambda}$ and hence

$$
\alpha \cdot \lambda+1>\left\|X_{\lfloor s\rfloor}\right\|_{\mathrm{W}} \geq \sup \{\alpha \cdot \eta+1 \mid \eta<\lambda\}
$$

that is, $\left\|X_{\lfloor s\rfloor}\right\|_{\mathrm{W}}=\alpha \cdot \lambda$. Since $\lambda$ is countable, then $X_{\lfloor s\rfloor}$ is self-dual and hence $\boldsymbol{T}\left(X_{\lfloor s\rfloor}\right)$ is well-founded. Therefore

$$
\varphi(s)=\lambda \text { limit } \Longrightarrow\left\|X_{\lfloor s\rfloor}\right\|_{\mathrm{W}}=\alpha \cdot \lambda \text { and } \boldsymbol{T}\left(X_{\lfloor s\rfloor}\right) \text { is well-founded. }
$$

Set

$$
C_{s}= \begin{cases}s^{\frown} X_{\lfloor s\rfloor} & \text { if } \varphi(s)=1, \\ \boldsymbol{r}_{T_{\eta}}^{-1}\left(X \cap\left(\left[T_{\eta}\right] \backslash\left[T_{\eta+1}\right]\right)\right) & \text { if } \varphi(s)=\eta+1>1, \\ \emptyset & \text { otherwise, }\end{cases}
$$

where $\boldsymbol{r}_{T_{\eta}}$ is the canonical retraction of $\mathbb{R}$ onto $\left[T_{\eta}\right]$. Therefore the definition of the $C_{s}$ depend only on the set $X$ and on the norm $\varphi$. The range of $\varphi$ need not be $\xi_{X}+1 \backslash\{0\}$, since it may happen that $T_{\eta}=T_{\eta+1}$ for some $\eta$.

Claim 25. Suppose $\lambda$ is limit, and $T_{\eta}=T_{\bar{\eta}}$ for all $\bar{\eta} \leq \eta<\lambda$. Then $T_{\lambda}=T_{\bar{\eta}}$.

Proof of the Claim. Suppose $T_{\lambda} \neq T_{\bar{\eta}}=\bigcap_{\eta<\lambda} T_{\eta}$. Let $s \in T_{\bar{\eta}} \cap \partial T_{\lambda}$ and $x$ be a real such that $s^{\frown} x \in\left[T_{\bar{\eta}}\right]$. Then $\forall n\left(\varphi\left(s^{\frown}(x\lceil n))=\lambda\right)\right.$, hence $x$ is a branch of $\boldsymbol{T}\left(X_{\lfloor s\rfloor}\right)$, a contradiction by (39).

Let $D$ be the closure of $\operatorname{ran}(\varphi)$. Since $\varphi(\emptyset)=\xi_{X}$, the order type of $D$ is a successor ordinal $\tilde{\xi}_{X}+1$. Let $h_{X}: D \rightarrow \tilde{\xi}_{X}+1$ be the collapsing function, let $\tilde{\varphi}=\tilde{\varphi}_{X}=h_{X} \circ \varphi$.

If $\varphi(s)$ is a successor ordinal, then it is a successor point of $D$, and by the Claim if $\varphi(s)$ is limit ordinal then so is $\tilde{\varphi}(s)$, hence we can conclude that $\varphi(s)$ is limit iff $\tilde{\varphi}(s)$ is limit. Letting $\left\langle\tilde{T}_{\eta} \mid \eta \leq \tilde{\xi}\right\rangle$ be the enumeration without repetitions of $\left\langle T_{\eta} \mid 1 \leq \eta \leq \xi\right\rangle$, we have that

$$
C_{s}= \begin{cases}s^{\frown} X_{\lfloor s\rfloor} & \text { if } \tilde{\varphi}(s)=0, \\ \boldsymbol{r}_{\tilde{T}_{\eta}}^{-1}\left(X \cap\left(\left[\tilde{T}_{\eta}\right] \backslash\left[\tilde{T}_{\eta+1}\right]\right)\right) & \text { if } \tilde{\varphi}(s)=\eta+1, \\ \emptyset & \text { otherwise. }\end{cases}
$$

Let $W_{X}$ be the pre-well-order induced by $\varphi$ or, equivalently, by $\tilde{\varphi}$. The main point of switching from $\varphi$ to $\tilde{\varphi}$ is that the latter, unlike the former, can be recovered from $W_{X}$. Thus $C_{s}$ depend only on $X$ and $W_{X}$, which are both sets of reals. By Lemma 5 , each $C_{s} \in \mathscr{P}^{(\alpha+1)}(\mathbb{R})$, so is enough to show that $X$ can be recovered from $W_{X}$ and from the sequence $\mathcal{C}_{X}=\left\langle C_{s} \mid s \in{ }^{<\omega} \omega\right\rangle$. Suppose $W_{X}=W_{Y}$ (and hence $\left.\tilde{\varphi}_{X}=\tilde{\varphi}_{Y}=\tilde{\varphi}\right)$, and $\mathcal{C}_{X}=\mathcal{C}_{Y}=\left\langle C_{s} \mid s \in{ }^{<\omega} \omega\right\rangle$. This implies that $\tilde{\xi}_{X}=\tilde{\xi}_{Y}=\tilde{\xi}$ and that the sequence $\left\langle\tilde{T}_{\eta} \mid \eta \leq \tilde{\xi}\right\rangle$ is the same for $X$ and $Y$. Let $x$ be a real. Since $\tilde{\varphi}\left(x\lceil n) \geq \tilde{\varphi}\left(x\lceil n+1)\right.\right.$, there is an $n_{0}$ and a $\gamma$ such that $\tilde{\varphi}(x \uparrow n)=\gamma$, for all $n \geq n_{0}$. If $\gamma$ is limit, then (39) implies that $\forall n \geq n_{0}\left(\left\|X X_{\lfloor x \uparrow n\rfloor}\right\|_{\mathrm{W}}=\alpha \cdot h_{X}^{-1}(\gamma)\right)$, 
where $h_{X}: \operatorname{ran}\left(\varphi_{X}\right) \rightarrow \tilde{\xi}_{X}+1$ is the collapsing function defined above. Hence $x$ would be a branch of $\left(x\left\lceil n_{0}\right)^{\wedge} \boldsymbol{T}\left(X_{\left\lfloor x\left\lceil n_{0}\right\rfloor\right.}\right)\right.$, contradicting (39). Therefore $\gamma=\eta+1$, that is $\forall n \geq n_{0}\left(x\left\lceil n \in \tilde{T}_{\eta} \backslash \tilde{T}_{\eta+1}\right)\right.$, which implies $x \in\left[\tilde{T}_{\eta}\right] \backslash\left[\tilde{T}_{\eta+1}\right]$ and $x=\boldsymbol{r}_{\tilde{T}_{\eta}}(x)$, and hence

$$
x \in X \Longleftrightarrow x \in C_{x\left\lceil n_{0}\right.} \Longleftrightarrow x \in Y,
$$

that is, $X=Y$. Therefore the map $X \mapsto\left(W_{X}, \mathrm{C}_{X}\right)$ is injective, as required.

Suppose now $A$ is non-self-dual. Then $B=A \oplus \neg A$ is self-dual and of rank $\beta=\alpha+1$. The result follows from Lemma 20, from the arguments above with $B$ and $\beta$ replacing $A$ and $\alpha$, and by observing that $\beta \cdot \omega_{1}=\alpha \cdot \omega_{1}$. This finishes the proof.

Corollary 26. If either $3<\alpha \leq \omega_{1}$ or else $\omega_{1}^{\xi}+1<\alpha \leq \omega_{1}^{\xi+1}<\Theta$ and $\xi>0$, then $\left|\mathscr{P}^{(\alpha)}(\mathbb{R})\right|$ is not a cardinality level.

In particular $\boldsymbol{\Delta}_{2}^{0}=\mathscr{P}^{\left(\omega_{1}\right)}(\mathbb{R})$ has the same cardinality as $\boldsymbol{\Delta}_{1}^{0}=\mathscr{P}^{(3)}(\mathbb{R})$, which has cardinality $|\mathbb{R}|$. Since $\boldsymbol{\Sigma}_{2}^{0} \cup \boldsymbol{\Pi}_{2}^{0}=\mathscr{P}^{\left(\omega_{1}+1\right)}(\mathbb{R})$ is a cardinality level by [Hjo98], it is tempting to conjecture that cardinality levels should occur exactly at Wadge rank $\omega_{1}^{\xi}+1$; or $\omega_{1}^{\xi}$ when $\xi$ is limit. The main result of this paper shows that this is indeed the case.

Theorem 1. Assume $\mathrm{AD}+\mathrm{DC}(\mathbb{R})$. Then $\mathscr{P}^{(\alpha)}(\mathbb{R})$ is a cardinality level iff

$$
\exists \xi<\Theta\left(\alpha=\omega_{1}^{\xi}+1 \vee\left(\alpha=\omega_{1}^{\xi} \& \xi \text { is limit }\right)\right) .
$$

This generalizes the results in [Hjo98] and [Hjo02] as well as the second author's results mentioned in the introduction.

Corollary 27. The following are cardinality pointclasses:

- $\boldsymbol{\Sigma}_{\alpha}^{0}$ (and hence $\boldsymbol{\Pi}_{\alpha}^{0}$ ) for $2 \leq \alpha<\omega_{1}$,

- $\boldsymbol{\Delta}_{1}^{0}$ and $\boldsymbol{\Delta}_{\alpha}^{0}$, for $3 \leq \alpha<\omega_{1}$,

- $\boldsymbol{\Sigma}_{n}^{1}$ (and hence $\boldsymbol{\Pi}_{n}^{1}$ ) for $1 \leq n<\omega$,

- $\boldsymbol{\Delta}_{n}^{1}$ for $1 \leq n<\omega$.

Proof. It is clear that $\boldsymbol{\Delta}_{1}^{0}$ is a cardinality pointclass, and from (18) it follows that $\left\|\boldsymbol{\Sigma}_{2}^{0}\right\|_{\mathrm{W}}=\omega_{1}+1$, hence $\boldsymbol{\Sigma}_{2}^{0}$ and $\boldsymbol{\Pi}_{2}^{0}$ are cardinality pointclasses. Suppose $\boldsymbol{\Sigma}$ is $\boldsymbol{\Sigma}_{\alpha}^{0}$ with $3 \leq \alpha$ or $\boldsymbol{\Sigma}_{n}^{1}$ : then $\boldsymbol{\Sigma}$ and $\breve{\boldsymbol{\Sigma}}$ satisfy the hypotheses of Lemma 8 , hence by (21) their Wadge rank is $\omega_{1}^{\xi}+1$ with $\xi$ limit of uncountable cofinality. (To show that if $C_{n}$ are closed and disjoint and $A_{n} \in \Pi_{\alpha}^{0}$, then $\bigcup_{n}\left(C_{n} \cap A_{n}\right) \in \Pi_{\alpha}^{0}$ argue as follows: if $A_{n}=\bigcap_{i} A_{n}^{i}$ with $A_{n}^{i} \in \Sigma_{\beta_{i}}^{0}$ with $2 \leq \beta_{i}<\alpha$, then $\bigcup_{n}\left(C_{n} \cap A_{n}\right)=\bigcap_{i}\left(\bigcup_{n}\left(C_{n} \cap A_{n}^{i}\right)\right)$.) Then $\boldsymbol{\Delta}=\boldsymbol{\Gamma} \cap \breve{\boldsymbol{\Gamma}}$ has rank $\omega_{1}^{\xi}$ with $\xi$ limit of uncountable cofinality, hence it is also a cardinality pointclass.

Before we start proving Theorem 1, let us make a few preliminary observations.

If $\Gamma$ is a pointclass generated by some set $A$, then there is a surjection $\mathbb{R} \rightarrow \Gamma$, since every continuous $\Phi:{ }^{<\omega} \omega \rightarrow{ }^{<\omega} \omega$ (and hence every continuous $f: \mathbb{R} \rightarrow \mathbb{R}$ ) can be coded by a real. As the principal pointclasses are cofinal in the ordering under inclusion, then every pointclass is the surjective image of $\mathbb{R}$. We will call any 
surjection $\pi: \mathbb{R} \rightarrow \Gamma$ a coding of $\Gamma$; more generally, if $\mathcal{X}$ is any non-empty set-not necessarily a pointclass - and $C$ is a non-empty $\mathbf{G}_{\delta}$ subset of $\mathbb{R}$, and $\pi: C \rightarrow \mathcal{X}$ is a surjection, then we will say that $\pi$ is a coding for $\mathcal{X}$ and that $X$ can be coded by reals. There are two kinds of codings for non-self-dual pointclasses $\Gamma$ that will be used: the first is $\mathbb{R} \rightarrow \boldsymbol{\Gamma}, x \mapsto \boldsymbol{\ell}_{x}^{-1}$ " $A$, where $A$ is $\boldsymbol{\Gamma}$-complete, and $\boldsymbol{\ell}_{x}$ is as in (3), and the second is $\mathcal{F} \rightarrow \boldsymbol{\Gamma}, x \mapsto \boldsymbol{f}_{x}^{-1}$ " $A$, where $\boldsymbol{\Gamma}$ is $\natural$-closed, $A^{\natural}$ is $\boldsymbol{\Gamma}$-complete, and $\boldsymbol{f}_{x}$ is as in (35). The next result says that an injection between pointclasses lifts to a continuous map defined on a comeager subset of the coding set.

Proposition 28. Suppose $\pi_{1}: C_{1} \rightarrow X_{1}$ and $\pi_{2}: C_{2} \rightarrow X_{2}$ are codings and suppose $F: X_{1} \longmapsto X_{2}$ is an injection. Then there is a set $\mathcal{G} \subseteq C_{1}$ which is comeager in $C_{1}$, and a continuous function $\hat{F}: \mathcal{G} \rightarrow C_{2}$ which induces $F \uparrow\left\{\pi_{1}(x) \mid x \in \mathcal{G}\right\}$ in the following sense:

$$
\forall x, y \in \mathcal{G}\left(\pi_{1}(x)=\pi_{1}(y) \Longleftrightarrow \pi_{2}(\hat{F}(x))=\pi_{2}(\hat{F}(y))\right)
$$

and

$$
\forall x \in \mathcal{G}\left(F\left(\pi_{1}(x)\right)=\pi_{2}(\hat{F}(x))\right) .
$$

In other words, the diagram

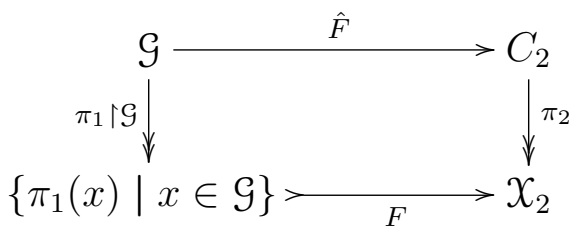

commutes.

Proof. Let $\Phi=\left\{(x, y) \in C_{1} \times C_{2} \mid F\left(\pi_{1}(x)\right)=\pi_{2}(y)\right\}$. Then $\forall x \in C_{1} \exists y \in$ $C_{2}(x, y) \in \Phi$. By $* *$-uniformization, there is a $\mathcal{G} \subseteq C_{1}$ comeager in $C_{1}$, and a function $\hat{F}: \mathcal{G} \rightarrow C_{2}$ uniformizing $\Phi$, that is $\forall x \in \mathcal{G}(x, \hat{F}(x)) \in \Phi$. Since AD implies that every function is continuous on a comeager set, we may assume $\hat{F}$ is continuous on $\mathcal{G}$.

Proof of Theorem 1. By Corollary 26, it is enough to show that for all $\xi<\Theta$

$$
\left|\mathscr{P}^{\left(\omega_{1}^{\xi}\right)}(\mathbb{R})\right|<\left|\mathscr{P}^{\left(\omega_{1}^{\xi}+1\right)}(\mathbb{R})\right|
$$

and

$$
\left|\mathscr{P}^{\left(\omega_{1}^{\gamma}\right)}(\mathbb{R})\right|<\left|\mathscr{P}^{\left(\omega_{1}^{\xi}\right)}(\mathbb{R})\right| \quad \text { if } \gamma<\xi \text { and } \xi \text { limit. }
$$

We will focus on the first inequality, since the second follows at once from it. Towards a contradiction, suppose that for some $\xi<\Theta$ we have that $\left|\mathscr{P}^{\left(\omega_{1}^{\xi}\right)}(\mathbb{R})\right|=$ $\left|\mathscr{P}^{\left(\omega_{1}^{\xi}+1\right)}(\mathbb{R})\right|$. If $\xi=0$ we reach a contradiction at once, as $\mathscr{P}^{(2)}(\mathbb{R})=\{\emptyset, \mathbb{R}\}$ and $\mathscr{P}^{(1)}(\mathbb{R})=\emptyset$, so we may assume that $\xi>0$. We must consider three cases.

Case 1: $\xi$ is a successor ordinal. 
By Lemma 16 there is a set $A_{1} \subseteq{ }^{\omega} 2$ such that $\left\|A_{1}\right\|_{\mathrm{W}}=\omega_{1}^{\xi}$ and such that

$$
\boldsymbol{\Gamma}_{1}=\left\{X \mid X \leq_{\mathrm{W}} A_{1}\right\}=\left\{\boldsymbol{f}_{x}^{-1} \text { " } A_{1} \mid x \in \mathfrak{F}\right\},
$$

where $x \mapsto \boldsymbol{f}_{x}$ is the parametrization of all $\natural$-functions described in (35). Let $A_{2}$ be non-self-dual and such that $\max \left\{\omega, \omega_{1}^{\xi-1}\right\} \leq\left\|A_{2}\right\|_{\mathrm{W}}<\omega_{1}^{\xi}$. By $(9)$

$$
\Gamma_{2}=\left\{X \mid X \leq_{\mathrm{W}} A_{2}\right\}=\left\{\boldsymbol{\ell}_{x}^{-1} \text { “ } A_{2} \mid x \in \mathbb{R}\right\} .
$$

Since $\left|\boldsymbol{\Gamma}_{2}\right|=\left|\mathscr{P}^{\left(\omega_{1}^{\xi}\right)}(\mathbb{R})\right|$ and $\left|\boldsymbol{\Gamma}_{1}\right|=\left|\mathscr{P}^{\left(\omega_{1}^{\xi}+1\right)}(\mathbb{R})\right|$ by Theorem 24 and Corollary 19, we may assume that there is an injective function $F: \Gamma_{1} \longmapsto \Gamma_{2}$. Let $\pi_{1}: \mathcal{F} \rightarrow \boldsymbol{\Gamma}_{1}$, $\pi_{1}(x)=\boldsymbol{f}_{x}^{-1}$ " $A_{1}$, and and $\pi_{2}: \mathbb{R} \rightarrow \boldsymbol{\Gamma}_{2}, \pi_{2}(x)=\boldsymbol{\ell}_{x}^{-1}$ " $A_{2}$. By Proposition 28 there is a comeager $\mathcal{G} \subseteq \mathcal{F}$ and a continuous $\hat{F}: \mathcal{G} \rightarrow \mathbb{R}$ which induces $F$, that is

$$
\begin{aligned}
F \uparrow\left\{\boldsymbol{f}_{x}^{-1} " A_{1} \mid x \in \mathcal{G}\right\}:\left\{\boldsymbol{f}_{x}^{-1} " A_{1} \mid x \in \mathcal{G}\right\} & \longmapsto \boldsymbol{\Gamma}_{2}, \\
\boldsymbol{f}_{x}^{-1} " A_{1} & \mapsto \boldsymbol{\ell}_{\hat{F}(x)}^{-1} " A_{2} .
\end{aligned}
$$

We need the following

Theorem 29. Suppose $\mathcal{K} \subseteq \mathcal{F}$ is non-meager in $\mathcal{F}$. Then there is a continuous map $g:{ }^{\omega} 2 \rightarrow \mathcal{K}$, and a real $\bar{z} \in \mathbb{R}$ such that:

$$
\begin{gathered}
\forall x \in{ }^{\omega} 2\left(\bar{z} \in \operatorname{dom}\left(\boldsymbol{f}_{g(x)}\right) \& \boldsymbol{f}_{g(x)}(\bar{z})=x\right), \\
\forall x, y \in{ }^{\omega_{2}} 2\left(\boldsymbol { f } _ { g ( x ) } \left\lceil\mathbb{R} \backslash\{\bar{z}\}=\boldsymbol{f}_{g(y)}\lceil\mathbb{R} \backslash\{\bar{z}\}) .\right.\right.
\end{gathered}
$$

Assuming Theorem 29 we can conclude the proof of Case 1: Let $\mathcal{K}=\mathcal{G}$, and let

$$
\begin{aligned}
C & =\left\{y \in \mathbb{R} \backslash\{\bar{z}\} \mid \exists x \in{ }^{\omega} 2\left(y \in \operatorname{dom}\left(\boldsymbol{f}_{g(x)}\right) \& \boldsymbol{f}_{g(x)}(y) \in A_{1}\right)\right\} \\
& =\left\{y \in \mathbb{R} \backslash\{\bar{z}\} \mid \forall x \in{ }^{\omega} 2\left(y \in \operatorname{dom}\left(\boldsymbol{f}_{g(x)}\right) \& \boldsymbol{f}_{g(x)}(y) \in A_{1}\right)\right\} \quad(\text { by }(43)) .
\end{aligned}
$$

For $x \in{ }^{\omega} 2$

$$
x \in A_{1} \Longrightarrow \boldsymbol{f}_{g(x)}^{-1} " A_{1}=C \cup\{\bar{z}\}, \quad \text { and } \quad x \notin A_{1} \Longrightarrow \boldsymbol{f}_{g(x)}^{-1} " A_{1}=C .
$$

Both $C$ and $C \cup\{\bar{z}\}$ belong to $\Gamma_{1}$, and since $\bar{z} \notin C$ and $F$ is injective, then $F(C) \triangle F(C \cup\{\bar{z}\}) \neq \emptyset$. Without loss of generality we may assume that there is a $y_{0} \in F(C \cup\{\bar{z}\}) \backslash F(C)$. If $x \in A_{1}$ then, by (44), $y_{0} \in F\left(\boldsymbol{f}_{g(x)}^{-1}\right.$ " $\left.A_{1}\right)=\boldsymbol{\ell}_{\hat{F}(g(x))}^{-1}$ " $A_{2}$ and hence $\boldsymbol{\ell}_{\hat{F}(g(x))}\left(y_{0}\right) \in A_{2}$. Similarly, $x \notin A_{1}$ implies $\boldsymbol{\ell}_{\hat{F}(g(x))}\left(y_{0}\right) \notin A_{2}$. Therefore $f:{ }^{\omega} 2 \rightarrow \mathbb{R}, x \mapsto \boldsymbol{\ell}_{\hat{F}(g(x))}\left(y_{0}\right)$, is continuous and $f^{-1} " A_{2}=A_{1}$, hence $A_{1} \leq{ }_{\mathrm{W}} A_{2}$ by Lemma 7 , contradicting the initial assumption that $\left\|A_{2}\right\|_{\mathrm{W}}<\left\|A_{1}\right\|_{\mathrm{W}}$. This concludes the proof of Case 1, assuming Theorem 29.

Notice that we have actually shown (modulo Theorem 29) that

Lemma 30. Suppose $A_{1}$ and $A_{2}$ are non-self-dual, with $\left(A_{1}\right)^{\natural} \equiv_{\mathrm{W}} A_{1} \subseteq{ }^{\omega} 2$, and let $\boldsymbol{\Gamma}_{i}=\left\{X \mid X \leq_{\mathrm{W}} A_{i}\right\}$, for $i=1,2$. Suppose $\pi_{1}: \mathcal{F} \rightarrow \boldsymbol{\Gamma}_{1}, x \mapsto \boldsymbol{f}_{x}^{-1} " A_{1}$, and $\pi_{2}: \mathbb{R} \rightarrow \boldsymbol{\Gamma}_{2}, x \mapsto \boldsymbol{\ell}_{x}^{-1}$ " $A_{2}$. Let $\mathcal{X}=\left\{\pi_{1}(x) \mid x \in \mathcal{K}\right\}$, where $\mathcal{K} \subseteq \mathcal{F}$ is non-meager, and suppose $\hat{F}: \mathcal{K} \rightarrow \mathbb{R}$ is a continuous function inducing an injection $F: \mathcal{X} \longmapsto \boldsymbol{\Gamma}_{2}$, $X \mapsto \pi_{2}(\hat{F}(x))$, for some/any $x \in \mathcal{K}$ such that $\pi_{1}(x)=X$. Then $A_{1} \leq_{\mathrm{W}} A_{2}$. 
Case 2: $\xi$ is limit and $\operatorname{cof}(\xi)>\omega$.

As in Case 1 , there is a set $A_{1} \equiv_{\mathrm{W}}\left(A_{1}\right)^{\natural}$ such that $\left\|A_{1}\right\|_{\mathrm{W}}=\omega_{1}^{\xi}$ and $A_{1} \subseteq{ }^{\omega} 2$ and let $\pi_{1}: \mathcal{F} \rightarrow\left\{X \mid X \leq_{\mathrm{W}} A_{1}\right\}=\Gamma_{1}, x \mapsto \boldsymbol{f}_{x}^{-1}$ " $A_{1}$. Let $\pi: \mathbb{R} \rightarrow \mathscr{P}^{\left(\omega_{1}^{\xi}\right)}(\mathbb{R})$. By Corollary 19, $\left|\boldsymbol{\Gamma}_{1}\right|=\left|\mathscr{P}^{\left(\omega_{1}^{\xi}+1\right)}(\mathbb{R})\right|$, so we may assume that there is an injection $F: \boldsymbol{\Gamma}_{1} \longmapsto \mathscr{P}^{\left(\omega_{1}^{\xi}\right)}(\mathbb{R})$ and therefore there is a comeager $\mathcal{G} \subseteq \mathcal{F}$ and a continuous $\hat{F}$ as in Proposition 28. For $\alpha<\omega_{1}^{\xi}$ let

$$
M_{\alpha}=\left\{x \in \mathcal{G} \mid\|\pi(\hat{F}(x))\|_{\mathrm{W}} \leq \alpha\right\} .
$$

Since $\bigcup_{\alpha<\omega_{1}^{\xi}} M_{\alpha}=\mathcal{G}$, then AD implies that $M_{\bar{\alpha}}$ is non-meager for some $\bar{\alpha}<\omega_{1}^{\xi}$. Let $\mathcal{K}=M_{\bar{\alpha}}$ and let $A_{2}$ be non-self-dual, of infinite rank and such that $\bar{\alpha}<\left\|A_{2}\right\|_{\mathrm{W}}<$ $\left\|A_{1}\right\|_{\mathrm{W}}$. Let $\boldsymbol{\Gamma}_{2}=\left\{X \mid X \leq_{\mathrm{W}} A_{2}\right\}$ and let $\pi_{2}: \mathbb{R} \rightarrow \boldsymbol{\Gamma}_{2}, x \mapsto \boldsymbol{\ell}_{x}^{-1}$ " $A_{2}$. Since $\left\{F\left(\boldsymbol{f}_{x}^{-1}\right.\right.$ " $\left.\left.A_{1}\right) \mid x \in \mathcal{K}\right\} \subseteq \boldsymbol{\Gamma}_{2}$, then $A_{1} \leq_{\mathrm{W}} A_{2}$ by Lemma 30: a contradiction.

Case 3: $\xi$ is limit and $\operatorname{cof}(\xi)=\omega$.

Choose an increasing sequence of successor ordinals $\xi_{n} \rightarrow \xi$ and sets $B_{n}$ of rank $\omega_{1}^{\xi_{n}}$. The $B_{n}$ 's are non-self-dual and by Lemma 16 they can be taken to be contained in $\omega_{2}$. By Lemma 23 we may assume there is an injection $F: y \longmapsto \mathscr{P}^{\left(\omega_{1}^{\xi}\right)}(\mathbb{R})$, where

$$
y=\left\{\left\langle X_{n} \mid n \in \omega\right\rangle \mid \forall n X_{n} \leq_{\mathrm{W}} B_{n}\right\} .
$$

Let $\pi: \mathbb{R} \rightarrow y$ be the coding given by

$$
x \mapsto\left\langle\ell_{x_{n}}^{-1 \text { " } B_{n}|n \in \omega\rangle,}\right.
$$

where $x \mapsto\left\langle x_{n} \mid n \in \omega\right\rangle, \mathbb{R} \rightarrow{ }^{\omega} \mathbb{R}$, is the standard homeomorphism. By Proposition 28 there is a comeager $\mathcal{G} \subseteq \mathbb{R}$ and a continuous $\hat{F}: \mathcal{G} \rightarrow \mathbb{R}$ inducing $F$. For each $n \in \omega$, let

$$
M_{n}=\left\{x \in \mathcal{G} \mid\|F(\pi(x))\|_{\mathrm{W}} \leq \omega_{1}^{\xi_{n}}\right\} .
$$

Arguing as in Case 2, there is $\bar{n}$ such that $M_{\bar{n}}$ is non-meager, and by the KuratowskiUlam Theorem there is a fixed sequence of reals $\left\langle x_{i}^{*} \mid i \neq \bar{n}+1\right\rangle$ such that

$$
\mathcal{K}=\left\{x \in \mathbb{R} \mid J\left(\left\langle x_{0}^{*}, \ldots, x_{\bar{n}}^{*}, x, x_{\bar{n}+2}^{*}, \ldots\right\rangle\right) \in M_{\bar{n}}\right\}
$$

is non-meager. Letting $A_{1}=B_{\bar{n}+1}, A_{2}=B_{\bar{n}}$, and $\mathcal{X}=\left\{\boldsymbol{\ell}_{x}^{-1}\right.$ " $\left.A_{1} \mid x \in \mathcal{K}\right\}$, then Lemma 30 implies that $A_{1} \leq_{\mathrm{W}} A_{2}$, contradicting our assumption.

This completes the proof of Theorem 1, granted Theorem 29.

\section{Proof of Theorem 29}

In this section we complete the proof of Theorem 1 by proving Theorem 29 .

Let us try first a simple-minded line of attack. Fix $x_{0} \in \mathcal{K}$ coding a sequence $\left\langle\left(T_{n}, \Phi_{n}\right) \mid n \in \omega\right\rangle \in \boldsymbol{F}$ as in (32), and let $\bar{z} \notin \bigcup_{n}\left[T_{n}\right]$. For any $x \in{ }^{\omega} 2$ let $T_{0}^{\prime}=\{\bar{z} \uparrow k \mid k \in \omega\}, \Phi_{0}^{\prime}(\bar{z} \uparrow k)=x \uparrow k$, and let $T_{n+1}^{\prime}=T_{n}$ and $\Phi_{n+1}^{\prime}=\Phi_{n}$. Let $g(x) \in \mathcal{F}$ be the unique real coding $\left\langle\left(T_{n}^{\prime}, \Phi_{n}^{\prime}\right) \mid n \in \omega\right\rangle$. It is not hard to see that $g:{ }^{\omega_{2}} \rightarrow \mathcal{F}$ is continuous and that $g(x)$ satisfies (42) and (43). The only problem is that $g(x)$ need not be in $\mathcal{K}$. In fact, it might be the case that the first tree of the generic sequence in $\boldsymbol{F}$ is not a singleton. This suggests that the choice of the initial sequence $\left\langle\left(T_{n}, \Phi_{n}\right) \mid n \in \omega\right\rangle$, of the real $\bar{z}$, and of the transformation 
procedure $\left\langle\left(T_{n}, \Phi_{n}\right) \mid n \in \omega\right\rangle \mapsto\left\langle\left(T_{n}^{\prime}, \Phi_{n}^{\prime}\right) \mid n \in \omega\right\rangle$ should be as generic as possible. The construction could be achieved using a Baire-category argument, but the level of details would be excessive. We prefer instead to use Cohen forcing: rather than specify in advance a countable family of dense $\mathbf{G}_{\delta}$ 's to be met, we work with fully generic objects, then observe that we only need to intersect countably many dense sets to perform the construction, so that the generic exists outright in V. A more elegant way to see this, is to appeal to absoluteness: if in the statement of Theorem 29 the set $\mathcal{K}$ is replaced with a $\mathcal{K}^{\prime} \subseteq \mathcal{K}$ such that $\mathcal{K}^{\prime}$ is $\mathbf{G}_{\delta}$ and non-meager in $\mathcal{F}$, the statement

$$
\exists \bar{z} \in \mathbb{R} \exists g:{ }^{\omega} 2 \rightarrow \mathcal{K}^{\prime} g \text { is continuous and satisfies (42) and (43) }
$$

is $\Sigma_{2}^{1}$, hence, by Shoenfield's absoluteness, a $g$ and a $\bar{z}$ as above can be found in $\mathrm{V}$.

Let $\mathbf{P}$ be the forcing notion that constructs with finite conditions a sequence $\left\langle\left(T_{n}, \Phi_{n}\right) \mid n \in \omega\right\rangle \in \boldsymbol{F}$. Formally, $\mathbf{P}$ is the collection of all

$$
p=\left(\left\langle\tau_{n} \mid n \in A\right\rangle,\left\langle\varphi_{n} \mid n \in A\right\rangle,\left\langle K_{n, m} \mid n, m \in A \& n \neq m\right\rangle\right)
$$

such that

- $A \subset \omega$ is finite,

- $\tau_{n}$ is a function, $\operatorname{dom}\left(\tau_{n}\right)$ is a finite subset of $<\omega \omega, \operatorname{ran}\left(\tau_{n}\right) \subseteq\{0,1\}$, and $t_{n}=\tau_{n}^{-1}$ " $\{1\}$ is a non-empty finite tree on $\omega$,

- $\varphi_{n}: t_{n} \rightarrow{ }^{<\omega} \omega$ is Lipschitz,

- $K_{n, m}=K_{m, n} \in \omega$, and $s_{k} \in t_{n} \cap t_{m} \Longrightarrow k \leq K_{n, m}$,

where $\left\langle\boldsymbol{s}_{n} \mid n \in \omega\right\rangle$ is as in (2). For ease of notation, a condition $p$ as above will be denoted as

$$
p=\left\langle\tau_{n}, \varphi_{n}, K_{n, m}\right\rangle_{n, m \in A} .
$$

The ordering on $\mathbf{P}$ is given by

$$
\begin{aligned}
&\left\langle\tau_{n}, \varphi_{n}, K_{n, m}\right\rangle_{n, m \in A} \leq\left\langle\tau_{n}^{\prime}, \varphi_{n}^{\prime}, K_{n, m}^{\prime}\right\rangle_{n, m \in A^{\prime}} \Longleftrightarrow \\
& A \supseteq A^{\prime} \& \forall n, m \in A^{\prime}\left(n \neq m \Longrightarrow \tau_{n} \supseteq \tau_{n}^{\prime} \& \varphi_{n} \supseteq \varphi_{n}^{\prime} \& K_{n, m} \leq K_{n, m}^{\prime}\right) .
\end{aligned}
$$

The poset $\mathbf{P}$ is countable, so it is just Cohen forcing in disguise. We will use $A^{(p)}$, $\tau_{n}^{(p)}, \varphi_{n}^{(p)}$, etc. to denote the various items given by $p \in \mathbf{P}$, and when there is no danger of confusion the superscript will be dropped. The domain of a condition $p$ is defined to be

$$
\operatorname{dom}(p)=\bigcup_{n \in A} \operatorname{dom}\left(\tau_{n}\right) .
$$

If $G \subset \mathbf{P}$ is a filter, then each

$$
T_{n}=\bigcup_{p \in G} t_{n}^{(p)}
$$

is a tree on $\omega, \boldsymbol{s}_{k} \in T_{n} \cap T_{m} \Longrightarrow k \leq K_{n, m}^{(p)}$ for any $p \in G$ such that $n, m \in A^{(p)}$ and hence $T_{n} \cap T_{m}$ is finite, and each

$$
\Phi_{n}=\bigcup_{p \in G} \varphi_{n}^{(p)}: T_{n} \rightarrow^{<\omega} \omega
$$


is Lipschitz. Moreover if $G$ is sufficiently generic, then each $T_{n}$ is pruned and nonempty, and hence $\left\langle\left(T_{n}, \Phi_{n}\right) \mid n \in \omega\right\rangle \in \boldsymbol{F}$. Conversely, any such sequence yields a filter $G \subseteq \mathbf{P}$ as follows: for any $p=\left\langle\tau_{n}, \varphi_{n}, K_{n, m}\right\rangle_{n, m \in A} \in \mathbf{P}$, set

$$
p \sqsubset\left\langle\left(T_{n}, \Phi_{n}\right) \mid n \in \omega\right\rangle
$$

just in case for every $n, m \in A, n \neq m$ :

- $\forall u \in \operatorname{dom}\left(\tau_{n}\right)\left(\tau_{n}(u)=1 \Longleftrightarrow u \in T_{n}\right)$,

- $\Phi_{n} \uparrow t_{n}=\varphi_{n}$,

- $\forall k\left(\boldsymbol{s}_{k} \in T_{n} \cap T_{m} \Longrightarrow k \leq K_{n, m}\right)$,

and let

$$
G=\left\{p \in \mathbf{P} \mid p \sqsubset\left\langle\left(T_{n}, \Phi_{n}\right) \mid n \in \omega\right\rangle\right\} .
$$

Thus we will say that $\left\langle\left(T_{n}, \Phi_{n}\right) \mid n \in \omega\right\rangle \in \boldsymbol{F}$ is generic if $\exists p \in D p \sqsubset\left\langle\left(T_{n}, \Phi_{n}\right)\right|$ $n \in \omega\rangle$ for every dense $D \subseteq \mathbf{P}$.

If we fix a condition $p$ forcing the sequence to be in the set $\boldsymbol{j}^{-1}$ " $\mathcal{K}$, which is non-meager in $\boldsymbol{F}$ (see (34)), then by replacing $\mathbf{P}$ with its localization $\mathbf{P}_{p}$

$$
\mathbf{P}_{p}=\left\{p^{\prime} \in \mathbf{P} \mid p^{\prime} \leq p\right\},
$$

we may assume that every generic yields a $\downarrow$-function with code in $\mathcal{K}$. In other words, we may assume that

$$
\mathcal{K}=\mathcal{F}
$$

So let us fix a $G \subset \mathbf{P}$ generic over $\mathrm{V}$, and hence a $\mathbf{P}$-generic sequence

$$
\left\langle\left(T_{n}, \Phi_{n}\right) \mid n \in \omega\right\rangle \in \boldsymbol{F} .
$$

Let

$$
f_{n}:\left[T_{n}\right] \rightarrow \mathbb{R}, \quad y \mapsto \bigcup_{k} \Phi_{n}(y \uparrow k),
$$

be the Lipschitz function induced by $\Phi_{n}$ 's, and let

$$
f=\bigcup_{n} f_{n}: \bigcup_{n}\left[T_{n}\right] \rightarrow \mathbb{R},
$$

be the $\downarrow$-function defined by $G$. By genericity, each $\left[T_{n}\right]$ is nowhere dense, hence

$$
\bigcup_{n}\left[T_{n}\right] \text { is meager. }
$$

Working in $\mathrm{V}[G]$, a map

$$
\begin{aligned}
& \left\{(s, u) \in{ }^{<\omega} 2 \times{ }^{<\omega} \omega \mid \operatorname{lh}(s)=\operatorname{lh}(u)\right\} \rightarrow \boldsymbol{F} \\
& (s, u) \mapsto\left\langle\left(T_{n}^{(s, u)}, \Phi_{n}^{(s, u)}\right) \mid n \in \omega\right\rangle
\end{aligned}
$$

is constructed. For brevity we will say that $\left\langle\left(T_{n}^{(s, u)}, \Phi_{n}^{(s, u)}\right) \mid n \in \omega\right\rangle$ is the sequence given by $(s, u)$. The sequences given by the $(s, u)$ 's will be $\mathbf{P}$-generic sequences over V. Let

$$
f_{n}^{(s, u)}:\left[T_{n}^{(s, u)}\right] \rightarrow \mathbb{R}
$$

be the Lipschitz map induced by $\Phi_{n}^{(s, u)}$, and let

$$
f^{(s, u)}=\bigcup_{n} f_{n}^{(s, u)}: \bigcup_{n}\left[T_{n}^{(s, u)}\right] \rightarrow \mathbb{R}
$$


be the $q$-function given by $(s, u)$. Recall that $\boldsymbol{H}$ (defined in (8)) is a superset of $\boldsymbol{F}$, and it is a Polish space. Fix a compatible complete metric $d$ for it. Then the sequence

$$
k \mapsto\left\langle\left(T_{n}^{(x \uparrow k, z \nmid k)}, \Phi_{n}^{(x\lceil k, z \uparrow k)}\right) \mid n \in \omega\right\rangle \in \boldsymbol{F} \subset \boldsymbol{H}
$$

is a $d$-Cauchy for each pair $(x, z) \in{ }^{\omega} 2 \times \mathbb{R}$, and the limit

$$
\left\langle\left(T_{n}^{(x, z)}, \Phi_{n}^{(x, z)}\right) \mid n \in \omega\right\rangle \in \boldsymbol{H}
$$

is called the sequence given by $(x, z)$. It is not, in general, an element of $\boldsymbol{F}$ and $a$ fortiori need not be $\mathbf{P}$-generic, since it may happen that $\left[T_{0}^{(x, z)}\right]$ is not disjoint from some $\left[T_{n}^{(x, z)}\right]$. For $(x, z) \in{ }^{\omega} 2 \times \mathbb{R}$, let

$$
f_{n}^{(x, z)}:\left[T_{n}^{(x, z)}\right] \rightarrow \mathbb{R}
$$

be the Lipschitz function induced by $\Phi_{n}^{(x, z)}$, and if the $\left[T_{n}^{(x, z)}\right]$ 's are disjoint, let

$$
f^{(x, z)}=\bigcup_{n} f_{n}^{(x, z)}: \bigcup_{n}\left[T_{n}^{(x, z)}\right] \rightarrow \mathbb{R} .
$$

In this case, the construction will guarantee that

$$
f^{(x, z)} \uparrow(\mathbb{R} \backslash\{z\})=f \uparrow(\mathbb{R} \backslash\{z\}),
$$

where $f$ is as in (51), and that

$$
z \in\left[T_{0}^{(x, z)}\right] \quad \text { and } \quad f_{0}^{(x, z)}(z)=x .
$$

In fact we need to find a real $\bar{z}$ such that for all $x \in{ }^{\omega} 2$ the sequence $\left\langle\left(T_{n}^{(x, z)}, \Phi_{n}^{(x, z)}\right)\right|$ $n \in \omega\rangle$ is in $\boldsymbol{F}$. In other words, we must find a $\bar{z}$ such that for all $x \in{ }^{\omega} 2$ the sequence given by $(x, \bar{z})$ is $\mathbf{P}$-generic over $\mathrm{V}$. To achieve this we use forcing again. Let $\mathbf{Q} \in \mathrm{V}[G]$ be the Cohen forcing to add a new real $\bar{z}$. By $(52), \bar{z} \notin \bigcup_{n}\left[T_{n}\right]$. Let

$$
g(x)=\boldsymbol{j}\left(\left\langle\left(T_{n}^{(x, \bar{z})}, \Phi_{n}^{(x, \bar{z})}\right) \mid n \in \omega\right\rangle\right) \in \mathcal{F}
$$

where $\boldsymbol{j}$ is as in (34). The function $g:{ }^{\omega} 2 \rightarrow \mathbb{R}$ is continuous and

$$
\begin{gathered}
\boldsymbol{f}_{g(x)} \uparrow(\mathbb{R} \backslash\{\bar{z}\})=f \uparrow(\mathbb{R} \backslash\{\bar{z}\}), \\
z \in \operatorname{dom}\left(\boldsymbol{f}_{g(x), 0}\right) \quad \text { and } \quad \boldsymbol{f}_{g(x), 0}(\bar{z})=x,
\end{gathered}
$$

which imply conditions (42) and (43), and hence the theorem will be proved.

Here come the details. We will ensure that:

\section{Requirements:}

(A) $\bigcup_{n}\left[T_{n}^{(s, u)}\right]=\bigcup_{n}\left[T_{n}\right]$, and $\bigcup_{n} f_{n}^{(s, u)}=\bigcup_{n} f_{n}=f$.

(B) If $\operatorname{lh}(s)=\operatorname{lh}(u)=k+1$, then either

$$
\left\langle\left(T_{n}^{(s, u)}, \Phi_{n}^{(s, u)}\right) \mid n \in \omega\right\rangle=\left\langle\left(T_{n}^{(s \uparrow k, u \uparrow k)}, \Phi_{n}^{(s \uparrow k, u \uparrow k)}\right) \mid n \in \omega\right\rangle,
$$

or else there is a unique $j>0$ such that

$$
\left(T_{0}^{(s, u)} \neq T_{0}^{(s \uparrow k, u \uparrow k)} \& T_{j}^{(s, u)} \neq T_{j}^{(s \uparrow k, u \uparrow k)}\right) \vee\left(\Phi_{0}^{(s, u)} \neq \Phi_{0}^{(s \uparrow k, u \uparrow k)} \& \Phi_{j}^{(s, u)} \neq \Phi_{j}^{(s \uparrow k, u \uparrow k)}\right) .
$$


Moreover, in this second case, for $n \in\{0, j\}$ the tree $T_{n}^{(s, u)}$ may differ from $T_{n}^{(s \nmid k, u \nmid k)}$ and the map $\Phi_{n}^{(s, u)}$ may differ from $\Phi_{n}^{(s\lceil k, u \uparrow k)}$ only on the nodes extending $u$, that is

$$
\begin{aligned}
& \forall v \in{ }^{<\omega} \omega\left[\left(v \in T_{n}^{(s, u)} \triangle T_{n}^{(s \uparrow k, u \uparrow k)} \vee\right.\right. \\
& \left.\left.\quad\left(v \in T_{n}^{(s, u)} \cap T_{n}^{(s \uparrow k, u \uparrow k)} \& \Phi_{n}^{(s, u)}(v) \neq \Phi_{n}^{(s \uparrow k, u \uparrow k)}(v)\right)\right) \Longrightarrow v \supseteq u\right]
\end{aligned}
$$

and this is the first time that the $j$-th coordinate has been changed, that is

$$
\forall k^{\prime}<k\left(T_{j}^{\left(s \nmid k^{\prime}, u \nmid k^{\prime}\right)}=T_{j}^{(s \uparrow k, u \uparrow k)} \& \Phi_{j}^{\left(s \nmid k^{\prime}, u \uparrow k^{\prime}\right)}=\Phi_{j}^{(s \nmid k, u \uparrow k)}\right) .
$$

(C) $u \in T_{0}^{(s, u)}$ and $\Phi_{0}^{(s, u)}(u)=s$.

(D) $\left\langle\left(T_{n}^{(s, u)}, \Phi_{n}^{(s, u)}\right) \mid n<\omega\right\rangle$ depends only on $(s, u)$ and $\left\langle\left(T_{n}, \Phi_{n}\right) \mid n<\omega\right\rangle$, and therefore belongs to $\mathrm{V}[G]$.

(E) Each $\left\langle\left(T_{n}^{(s, u)}, \Phi_{n}^{(s, u)}\right) \mid n<\omega\right\rangle$ is $\mathbf{P}$-generic over $\mathrm{V}$.

We are ready to construct, by induction on $\operatorname{lh}(s)=\operatorname{lh}(u)$, the map in (53) satisfying the requirements $(\mathrm{A})-(\mathrm{E})$ above.

\section{The construction:}

The sequence given by $(\emptyset, \emptyset)$ is the original generic sequence in $(49)$, that is

$$
\left\langle\left(T_{n}^{(\emptyset, \emptyset)}, \Phi_{n}^{(\emptyset, \emptyset)}\right) \mid n<\omega\right\rangle=\left\langle\left(T_{n}, \Phi_{n}\right) \mid n<\omega\right\rangle .
$$

It is easy to verify that $(\mathrm{A})-(\mathrm{E})$ hold.

Suppose $\operatorname{lh}(s)=\operatorname{lh}(u)=k+1$, and suppose that the codes have been defined for all $\left(s^{\prime}, u^{\prime}\right)$ of length $\leq k$, and that satisfy $(\mathrm{A})-(\mathrm{E})$.

Case 1: $u \in T_{0}^{(s \uparrow k, u \uparrow k)}$ and $\Phi_{0}^{(s \uparrow k, u \uparrow k)}(u)=s$.

Then set

$$
\left\langle\left(T_{n}^{(s, u)}, \Phi_{n}^{(s, u)}\right) \mid n \in \omega\right\rangle=\left\langle\left(T_{n}^{(s \uparrow k, u \uparrow k)}, \Phi_{n}^{(s \uparrow k, u \uparrow k)}\right) \mid n \in \omega\right\rangle .
$$

Requirements (A)-(E) hold by the inductive assumption.

Case 2: otherwise, that is: $u \in T_{0}^{(s \uparrow k, u \uparrow k)} \Longrightarrow \Phi_{0}^{(s \uparrow k, u \uparrow k)}(u) \neq s$.

Since the sequence given by $(s \uparrow k, u \uparrow k)$ is $\mathbf{P}$-generic over $\mathrm{V}$ by $(\mathrm{E})$, a density argument implies there is a least $j>0$ such that $u \in T_{j}^{(s \nmid k, u \nmid k)}, \Phi_{j}^{(s \nmid k, u \uparrow k)}(u)=s$, and

$$
\forall k^{\prime}<k\left(T_{j}^{\left(s\left\lceil k^{\prime}, u \nmid k^{\prime}\right)\right.}=T_{j}^{(s \uparrow k, u \uparrow k)} \& \Phi_{j}^{\left(s\left\lceil k^{\prime}, u \uparrow k^{\prime}\right)\right.}=\Phi_{j}^{(s \uparrow k, u \uparrow k)}\right) .
$$

Define $\left\langle T_{n}^{(s, u)}, \Phi_{n}^{(s, u)} \mid n \in \omega\right\rangle$ as follows:

(i) $v \in T_{n}^{(s, u)} \Longleftrightarrow v \in T_{n}^{(s \nmid k, u \uparrow k)}$ and $\Phi_{n}^{(s, u)}(v)=\Phi_{n}^{(s \uparrow k, u \uparrow k)}(v)$ for all $v$ if $n \notin\{0, j\}$, and for $v \nsupseteq u$ if $n \in\{0, j\}$.

(ii) $v \in T_{0}^{(s, u)} \Longleftrightarrow v \in T_{j}^{(s \uparrow k, u \uparrow k)}$ and $\Phi_{0}^{(s, u)}(v)=\Phi_{j}^{(s \uparrow k, u \uparrow k)}(v)$ for $v \supseteq u$.

(iii) $v \in T_{j}^{(s, u)} \Longleftrightarrow v \in T_{0}^{(s \nmid k, u \uparrow k)}$ and $\Phi_{j}^{(s, u)}(v)=\Phi_{0}^{(s \uparrow k, u \uparrow k)}(v)$ for $v \supseteq u$. 
Thus the move from the sequence given by $(s \uparrow k, u \uparrow k)$ to the sequence given by $(s, u)$ involves interchanging the $u$-cone of $T_{0}$ with the $u$-cone of $T_{j}$, and similarly for $\Phi$. (This interchanging is phrased precisely in clauses (ii) and (iii).) It is clear that a change of this kind maintains the requirements (A) and (D), and satisfies the requirement $(\mathrm{B})$. Moreover the change secures the requirement $(\mathrm{C})$ since $j$ was specifically chosen so that $u \in T_{j}^{(s \nmid k, u \uparrow k)}$ and $\Phi_{j}^{(s \nmid k, u \uparrow k)}(u)=s$. It remains to verify that requirement (E) holds for $\left\langle\left(T_{n}^{(s, u)}, \Phi_{n}^{(s, u)}\right) \mid n \in \omega\right\rangle$.

Claim 31. $\left\langle\left(T_{n}^{(s, u)}, \Phi_{n}^{(s, u)}\right) \mid n \in \omega\right\rangle$ is $\mathbf{P}$-generic over $\mathrm{V}$.

Proof. Say that a condition $p=\left\langle\tau_{n}, \varphi_{n}, K_{n, m}\right\rangle_{n, m \in A}$ in $\mathbf{P}$ is nice just in case that:

- $\tau_{n}, n \in A$, all have the same domain. Moreover they all have domain equal to $\left\{s_{i} \mid i<K\right\}$ for some $K \in \omega$ which we denote $K^{(p)}$.

- $K_{n, m} \leq K$ for all $n \neq m$, both in $A$.

- Each $K_{n, m}$ is minimal, in the sense that there is no $p^{\prime} \leq p$ with $K_{n, m}^{\left(p^{\prime}\right)}<K_{n, m}$. (Equivalently, $K_{n, m}=0$ or else $\tau_{n}$ and $\tau_{m}$ disagree on $s_{K_{n, m}-1}$.)

Notice that every condition can be strengthened to a nice condition. The set of nice conditions is therefore dense in $\mathbf{P}$. Notice further that a nice condition is completely determined by $\left\langle\tau_{n}, \varphi_{n}\right\rangle_{n \in A}$, since $K_{n, m}$ is precisely specified by the final clause.

For each nice condition $p=\left\langle\tau_{n}, \varphi_{n}, K_{n, m}\right\rangle_{n, m \in A}$ let $\pi(p)$ be the nice condition $p^{*}=\left\langle\tau_{n}^{*}, \varphi_{n}^{*}, K_{n, m}^{*}\right\rangle_{n, m \in A}$ determined by:

(i) $v \in \tau_{n}^{*} \Longleftrightarrow v \in \tau_{n}$ and $\varphi_{n}^{*}(v)=\varphi_{n}(v)$ for all $v$ if $n \notin\{0, j\}$, and for $v \nsupseteq u$ if $n \in\{0, j\}$.

(ii) $v \in \tau_{0}^{*} \Longleftrightarrow v \in \tau_{j}$ and $\varphi_{0}^{*}(v)=\varphi_{j}(v)$ for $v \supseteq u$.

(iii) $v \in \tau_{j}^{*} \Longleftrightarrow v \in \tau_{0}$ and $\varphi_{j}^{*}(v)=\varphi_{0}(v)$ for $v \supseteq u$.

(These clauses, and the demand that $p^{*}$ be nice, completely determine $p^{*}$.)

It's easy to check that $q \leq p \Longrightarrow \pi(q) \leq \pi(p)$. Moreover it's clear that $\pi$ is its own inverse, and therefore $\pi$ is an isomorphism on nice conditions.

$\left\langle T_{n}^{(s, u)}, \Phi_{n}^{(s, u)} \mid n \in \omega\right\rangle$ and $\pi$ are defined so that $p \sqsubset\left\langle\left(T_{n}^{(s\lceil k, u \uparrow k)}, \Phi_{n}^{(s\lceil k, u \uparrow k)}\right) \mid n \in \omega\right\rangle$ iff $\pi(p) \sqsubset\left\langle\left(T_{n}^{(s, u)}, \Phi_{n}^{(s, u)}\right) \mid n \in \omega\right\rangle$. Using this, the fact that $\pi$ is an isomorphism on nice conditions, the fact that the nice conditions are dense in $\mathbf{P}$, and the genericity of $\left\langle\left(T_{n}^{(s\lceil k, u \uparrow k)}, \Phi_{n}^{(s \uparrow k, u \uparrow k)}\right) \mid n \in \omega\right\rangle$, it follows that $\left\langle\left(T_{n}^{(s, u)}, \Phi_{n}^{(s, u)}\right) \mid n \in \omega\right\rangle$ is generic.

Cases 1 and 2 above complete the construction of the map $(s, u) \mapsto\left\langle\left(T_{n}^{(s, u)}, \Phi_{n}^{(s, u)}\right)\right|$ $n \in \omega\rangle$ as in (53), satisfying (A)-(E).

By (B), for every pair $(s, u)$ of length $k+1$, for every $n \in \omega$, and for every $v \in<^{<\omega} \omega$,

$$
\operatorname{lh}(v) \leq k \Longrightarrow\left(\left(v \in T_{n}^{(s, u)} \Longleftrightarrow v \in T_{n}^{(s \uparrow k, u \uparrow k)}\right) \& \Phi_{n}^{(s, u)}(v)=\Phi_{n}^{(s \uparrow k, u \uparrow k)}(v)\right),
$$

hence for any $(x, z) \in{ }^{\omega} 2 \times \mathbb{R}$ let

$$
\begin{aligned}
T_{n}^{(x, z)} & =\left\{v \in{ }^{<\omega} \omega \mid \exists k \geq \operatorname{lh}(v) v \in T_{n}^{(x\lceil k, z \nmid k)}\right\} \\
& =\left\{v \in{ }^{<\omega} \omega \mid \forall k \geq \operatorname{lh}(v) v \in T_{n}^{(x\lceil k, z \nmid k)}\right\} .
\end{aligned}
$$


Then $T_{n}^{(x, z)}$ is a pruned tree, and $\Phi_{n}^{(x, z)}: T_{n}^{(x, z)} \rightarrow{ }^{<\omega} \omega$ is Lipschitz, where for $v \in T_{n}^{(x, z)}$ and $u \in<^{<\omega} 2$ we set

$$
\begin{aligned}
\Phi_{n}^{(x, z)}(v)=u & \Longleftrightarrow \exists k \geq \operatorname{lh}(v) \Phi_{n}^{(x\lceil k, z\lceil k)}(v)=u \\
& \Longleftrightarrow \forall k \geq \operatorname{lh}(v) \Phi_{n}^{(x\lceil k, z \mid k)}(v)=u .
\end{aligned}
$$

Therefore the map

$$
\omega_{2} \times \mathbb{R} \rightarrow \boldsymbol{H}, \quad(x, z) \mapsto\left\langle\left(T_{n}^{(x, z)}, \Phi_{n}^{(x, z)}\right) \mid n \in \omega\right\rangle
$$

is continuous. Let $f_{n}^{(x, z)}:\left[T_{n}^{(x, z)}\right] \rightarrow \mathbb{R}$ be the Lipschitz function induced by $\Phi_{n}^{(x \uparrow k, z \uparrow k)}$. By $(\mathrm{C}), z \in\left[T_{0}^{(x, z)}\right]$ and $f_{0}^{(x, z)}(z)=x$, so (55) holds.

Claim 32. Suppose $z \notin \bigcup_{n}\left[T_{n}\right]$. Then

(a) $n>0 \Longrightarrow z \notin\left[T_{n}^{(x, z)}\right]$,

(b) $\bigcup_{n}\left[T_{n}^{(x, z)}\right]=\{z\} \cup \bigcup_{n}\left[T_{n}\right]$,

(c) $n \neq m \Longrightarrow\left[T_{n}^{(x, z)}\right] \cap\left[T_{m}^{(x, z)}\right]=\emptyset$.

Proof. (a) If $\forall k\left(T_{n}^{(x \uparrow k, z \nmid k)}=T_{n}^{(x \nmid k+1, z \nmid k+1)}\right)$ then $T_{n}^{(x, z)}=T_{n}$, hence the condition follows from $z \notin \bigcup_{n}\left[T_{n}\right]$. Otherwise, let $k$ be such that $T_{n}^{(x \uparrow k, z \nmid k)} \neq T_{n}^{(x \uparrow k+1, z \nmid k+1)}$. Notice that $k$ is unique by (B). Again by (B), $T_{n}^{(x, z)}=T_{n}^{(x \uparrow k+1, z \uparrow k+1)}$, and since $\left[T_{n}^{(x \nmid k+1, z \nmid k+1)}\right] \subseteq \bigcup_{m}\left[T_{m}\right]$ by $(\mathrm{A})$, the result follows.

(b) Let $y \neq z$. By $(\mathrm{B})$

$$
y \uparrow k \neq z \uparrow k \Longrightarrow \forall n\left(y \in\left[T_{n}^{(x, z)}\right] \Longleftrightarrow y \in\left[T_{n}^{(x \uparrow k, z \uparrow k)}\right]\right)
$$

and therefore $y \in \bigcup_{n}\left[T_{n}^{(x, z)}\right] \Longleftrightarrow y \in \bigcup_{n}\left[T_{n}\right]$ by $(\mathrm{A})$.

(c) Towards a contradiction, suppose $y \in\left[T_{n}^{(x, z)}\right] \cap\left[T_{m}^{(x, z)}\right]$. By part (a) $y \neq z$ so by (57) above, $y \in\left[T_{n}^{(x \nmid k, z \nmid k)}\right] \cap\left[T_{m}^{(x\lceil k, z \nmid k)}\right]$ for all sufficiently large $k$, contradicting the fact that the $\left[T_{n}^{(x \nmid k, z \nmid k)}\right]$ are disjoint.

Therefore if $z \notin \bigcup_{n}\left[T_{n}\right]$, then the $\left[T_{n}^{(x, z)}\right]$ 's are disjoint, hence the -function $f^{(x, z)}=\bigcup_{n} f_{n}^{(x, z)}: \bigcup_{n}\left[T_{n}^{(x, z)}\right] \rightarrow \mathbb{R}$ is defined. The next Claim shows that (54) holds.

Claim 33. Suppose $z \notin \bigcup_{n}\left[T_{n}\right]$. Then $f^{(x, z)} \uparrow \bigcup_{n}\left[T_{n}\right]=f$.

Proof. Let $y \in\left[T_{n}\right]$ and let $k$ be least such that $y \uparrow k \neq z \uparrow k$. By condition (B) $y \in\left[T_{m}^{(x \nmid k, z \nmid k)}\right]$ for some $m$, and hence $f_{m}^{(x, z)}(y)=f_{m}^{(x \nmid k, z \nmid k)}(y)=f(y)$ by $(\mathrm{A})$ and (B).

Let $\mathbf{Q}$ be the usual Cohen forcing to add an element of $\mathbb{R}$ : $\mathbf{Q}={ }^{<\omega} \omega$ and the ordering is reverse inclusion, $u^{\prime} \leq u \Longleftrightarrow u^{\prime} \supseteq u$. Let $\bar{z}$ be Q-generic over $\mathrm{V}[G]$. Then by $(52) \bar{z} \notin \bigcup_{n}\left[T_{n}\right]$. In order to complete the proof it is enough to show that $g(x)$, the code for $f^{(x, \bar{z})}$, is in $\mathcal{K}\left(=\mathcal{F}\right.$ by (48)) for every $x \in{ }^{\omega_{2}}$. Therefore it is enough to verify that $\left\langle\left(T_{n}^{(x, \bar{z})}, \Phi_{n}^{(x, \bar{z})}\right) \mid n \in \omega\right\rangle$ is $\mathbf{P}$-generic over $\mathrm{V}$, for every $x \in{ }^{\omega} 2$. 
We now step-back into $\mathrm{V}$.

Let $\left\langle\left(\dot{T}_{n}, \dot{\Phi}_{n}\right) \mid n \in \omega\right\rangle$ be the $\mathbf{P}$-name for the generic sequence of disjoint closed sets and Lipschitz functions, let $\dot{z}$ be the Q-name for the generic real, so that

$$
\left(\left\langle\left(\dot{T}_{n}, \dot{\Phi}_{n}\right) \mid n \in \omega\right\rangle, \dot{z}\right)
$$

can be construed as the canonical $\mathbf{P} \times \mathbf{Q}$-name for the generic object. For $(s, u) \in$ $<\omega_{2} \times{ }^{<\omega} \omega$ with $\operatorname{lh}(s)=\operatorname{lh}(u)$, let $\left\langle\left(\dot{T}_{n}^{(s, u)}, \dot{\Phi}_{n}^{(s, u)}\right) \mid n \in \omega\right\rangle$ be the P-name for $\left\langle\left(T_{n}^{(s, u)}, \Phi_{n}^{(s, u)}\right) \mid n \in \omega\right\rangle$. Let $\dot{x}$ be a $\mathbf{P} \times \mathbf{Q}$-name for an element of the Cantor space, and let $\left\langle\left(\dot{T}_{n}^{(\dot{x}, \dot{z})}, \dot{\Phi}_{n}^{(\dot{x}, \dot{z})}\right) \mid n \in \omega\right\rangle$ be the $\mathbf{P} \times \mathbf{Q}$-name for the sequence given by the interpretations of $\dot{x}$ and $\dot{z}$ in the generic extension. Let $\mathcal{D}=\{D \subseteq \mathbf{P} \mid D$ is dense $\}$ : by $(\mathrm{E})$

$$
\Vdash_{\mathbf{P}}\left\langle\left(\dot{T}_{n}^{(s, u)}, \dot{\Phi}_{n}^{(s, u)}\right) \mid n \in \omega\right\rangle \text { is } \check{\mathcal{D}} \text {-generic }
$$

and in order to finish the proof we must check that

$$
\Vdash_{\mathbf{P} \times \mathbf{Q}}\left\langle\left(\dot{T}_{n}^{(\dot{x}, \dot{z})}, \dot{\Phi}_{n}^{(\dot{x}, \dot{z})}\right) \mid n \in \omega\right\rangle \text { is } \check{\mathcal{D}} \text {-generic. }
$$

Towards a contradiction, suppose there are $D \in \mathcal{D}$ and $(p, u) \in \mathbf{P} \times \mathbf{Q}$ such that

$$
(p, u) \Vdash_{\mathbf{P} \times \mathbf{Q}}\left\langle\left(\dot{T}_{n}^{(\dot{x}, \dot{z})}, \dot{\Phi}_{n}^{(\dot{x}, \dot{z})}\right) \mid n \in \omega\right\rangle \text { does not meet } \check{D} .
$$

Let $k=\operatorname{lh}(u)$. By (58), for any $s \in{ }^{k} 2$ and any $p^{\prime} \in \mathbf{P}$ there are $p_{s}^{*} \in \mathbf{P}$ and $p_{s} \in D$ such that $p_{s}^{*} \leq p^{\prime}$ and

$$
p_{s}^{*} \Vdash_{\mathbf{P}} \check{p}_{s} \sqsubset\left\langle\left(\dot{T}_{n}^{(s, u)}, \dot{\Phi}_{n}^{(s, u)}\right) \mid n \in \omega\right\rangle .
$$

Applying this argument $2^{k}$-many times, for each $s \in{ }^{k} 2$ we can find $p_{s} \in D$ and $p_{s}^{*} \in \mathbf{P}$ satisfying (61) and such that the $p_{s}^{*}$ 's extend one another ${ }^{2}$ and each of them extends $p$. Let $p^{*}$ be the strongest of the $p_{s}^{*}$ s, and let $w=u^{\curlyvee} m$, where $m$ is large enough so that $w \notin \bigcup_{s \in k^{k}} \operatorname{dom}\left(p_{s}\right)$. (The domain of a condition is defined in (46).) Therefore, for any $s \in{ }^{k} 2$

$$
p^{*} \vdash_{\mathbf{P}} \check{p}_{s} \sqsubset\left\langle\left(\dot{T}_{n}^{(s, u)}, \dot{\Phi}_{n}^{(s, u)}\right) \mid n \in \omega\right\rangle .
$$

For any $n \in \omega$, for any $s^{\prime} \in{ }^{k+1} 2$, and any $(t, v) \in{ }^{<\omega} 2 \times<\omega \omega$ with $t \supset s^{\prime}, v \supseteq w$, and $\operatorname{lh}(t)=\operatorname{lh}(v)$,

$\Vdash_{\mathbf{P}} \dot{T}_{n}^{\left(s^{\prime}, w\right)}, \dot{\Phi}_{n}^{\left(s^{\prime}, w\right)}$ may differ from $\dot{T}_{n}^{(t, v)}, \dot{\Phi}_{n}^{(t, v)}$ only on nodes extending $w$ by (B), and since $w$ is not in the domain of any of the $p_{s}$ 's, then for each $s \in{ }^{k} 2$

$$
\begin{aligned}
p^{*} \vdash_{\mathbf{P}} \forall(t, v) \in{ }^{<\omega} 2 \times{ }^{<\omega} \omega(\operatorname{lh}(t)=\operatorname{lh}(v) \& t \supset \check{s} \& v & \supseteq \check{w} \Longrightarrow \\
\check{p_{s}} & \left.\sqsubset\left\langle\left(\dot{T}_{n}^{(t, v)}, \dot{\Phi}_{n}^{(t, v)}\right) \mid n \in \omega\right\rangle\right) .
\end{aligned}
$$

Since $p_{s} \in D$ then

$$
\left(p^{*}, w\right) \Vdash_{\mathbf{P} \times \mathbf{Q}}\left\langle\left(\dot{T}_{n}^{(\dot{x}, \dot{z})}, \dot{\Phi}_{n}^{(\dot{x}, \dot{z})}\right) \mid n \in \omega\right\rangle \text { meets } \check{D},
$$

contradicting $(60)$, as $\left(p^{*}, w\right) \leq(p, u)$. This completes the proof of Theorem 29 .

\footnotetext{
${ }^{2}$ This is why we restricted to $x \in{ }^{\omega} 2$, rather than $x \in \mathbb{R}$ : at each stage of the approximation we only need to worry about finitely many $s$.
} 


\section{Appendix}

Recall from (6) that PTr, the collection of all non-empty pruned trees on $\omega$, is (or better: can be identified with) a $\mathbf{G}_{\delta}$ subset of ${ }^{\omega} 2$. Hence ${ }^{\omega} \mathrm{P} T r$ can be construed as the Polish space of all codes for $\Sigma_{2}^{0}$ sets. Let $\pi_{1}:{ }^{\omega} \mathrm{PTr} \rightarrow \Sigma_{2}^{0}$

$$
\pi_{1}\left(\left\langle T_{n} \mid n<\omega\right\rangle\right)=\bigcup_{n}\left[T_{n}\right] .
$$

Similarly ${ }^{\omega \times 2} \mathrm{PTr}$ is Polish and $\pi_{2}:{ }^{\omega \times 2} \mathrm{PTr} \rightarrow 2-\Sigma_{2}^{0}$

$$
\pi_{2}\left(\left\langle T_{n, i} \mid(n, i) \in \omega \times 2\right\rangle\right)=\bigcup_{n}\left[T_{n, 0}\right] \backslash \bigcup_{n}\left[T_{n, 1}\right] .
$$

As in Proposition 28, a function $F:{ }^{\omega \times 2} \mathrm{PTr} \rightarrow{ }^{\omega} \mathrm{P} \operatorname{Tr}$ induces an injection of $2-\Sigma_{2}^{0}$ into $\Sigma_{2}^{0}$ iff

$$
\forall \vec{T}, \vec{S} \in{ }^{\omega \times 2} \operatorname{PTr}\left(\pi_{2}(\vec{T})=\pi_{2}(\vec{S}) \Longleftrightarrow \pi_{1}(F(\vec{T}))=\pi_{1}(F(\vec{S}))\right) .
$$

The map $2-\Sigma_{2}^{0} \longmapsto \Sigma_{2}^{0}, A \mapsto \pi_{1}(\vec{F}(\vec{T}))$ for some/any $\vec{T} \in{ }^{\omega \times 2} \mathrm{PTr}$ such that $\pi_{2}(\vec{T})=$ $A$, will be the injection induced by $F$.

We will sketch a proof of

Theorem 34. Assume AD. Then there is no $F:{ }^{\omega \times 2} \mathrm{PTr} \rightarrow{ }^{\omega} \mathrm{PTr}$ which induces an injection $2-\Sigma_{2}^{0} \longmapsto \Sigma_{2}^{0}$.

This was our first result and the techniques employed in its proof-once properly generalized-led to Theorem 1 . The proof of Theorem 34 rests on two intermediate results: the first is a weaker version of the theorem, Lemma 35 below, saying that no such $F$ can be continuous, and the second is a generalization to all $F$ 's.

Lemma 35. Assume ZF + DC. Then there is no continuous $F:{ }^{\omega \times 2} \mathrm{PTr} \rightarrow{ }^{\omega} \mathrm{PTr}$ which induces an injection of $2-\Sigma_{2}^{0}$ into $\Sigma_{2}^{0}$.

Proof. Since every real yields a pruned tree via the map $H: \mathbb{R} \longmapsto \operatorname{PTr}, x \mapsto\{x \uparrow$ $n \mid n \in \omega\}$, then ${ }^{\omega \times 2} \mathbb{R}$ can be embedded into ${ }^{\omega \times 2} \mathrm{P} \operatorname{Tr}$ via the map

$$
H_{2}:{ }^{\omega \times 2} \mathbb{R} \longmapsto{ }^{\omega \times 2} \mathrm{PTr} \quad\left\langle x_{n, i} \mid(n, i) \in \omega \times 2\right\rangle \mapsto\left\langle H\left(x_{n, i}\right) \mid(n, i) \in \omega \times 2\right\rangle .
$$

Therefore every $x \in{ }^{\omega \times 2} \mathbb{R}$ yields a set in $2-\Sigma_{2}^{0}$

$$
\begin{aligned}
D(2 ; x) & =\operatorname{Diff}\left(2 ; H_{2}(x)\right) \\
& =\left\{x_{n, 0} \mid n<\omega\right\} \backslash\left\{x_{n, 1} \mid n<\omega\right\}
\end{aligned}
$$

Note that $D(2 ; x)$ is indeed a countable set and hence an element of $\Sigma_{2}^{0}$, but we shall think of it as an element of $2-\Sigma_{2}^{0}$. Since $\Phi_{2}$ is continuous, it is enough to show that there is no continuous $F:{ }^{\omega \times 2} \mathbb{R} \rightarrow{ }^{\omega} \mathrm{PTr}$ such that for all $x, y \in{ }^{\omega \times 2} \mathbb{R}$

$$
D(2 ; x)=D(2 ; y) \Longleftrightarrow \bigcup_{n}\left[F(x)_{n}\right]=\bigcup_{n}\left[F(y)_{n}\right] .
$$

Towards a contradiction assume that such an $F$ exists. We will often blur the distinction between a countable sequence of reals $a \in{ }^{\omega \times 2} \mathbb{R}$ and the countable set of reals it defines, $\operatorname{ran}(a)=\left\{a_{n, i} \mid(n, i) \in \omega \times 2\right\}$. In particular the usual set theoretic operations and properties applied to elements of $\omega \times 2 \mathbb{R}$ are really meant to be applied 
to the sets of reals defined by them, so that $a \subseteq b, a \cup b, \ldots$ stand for $\left\{a_{n, i} \mid(n, i) \in\right.$ $\omega \times 2\} \subseteq\left\{b_{n, i} \mid(n, i) \in \omega \times 2\right\},\left\{a_{n, i} \mid(n, i) \in \omega \times 2\right\} \cup\left\{b_{n, i} \mid(n, i) \in \omega \times 2\right\}, \ldots$

Fix $a, b, c \in{ }^{\omega} \mathbb{R}$ pairwise disjoint sequences of reals, and assume that $c$ is dense (i.e., $\left\{c_{n} \mid n \in \omega\right\}$ is dense in $\mathbb{R}$ ). Let $x \in{ }^{\omega \times 2} \mathbb{R}$ be defined by

$$
x_{n, 0}=a_{n}, \quad y_{n, 0}=b_{n}, \quad x_{n, 1}=y_{n, 1}=c_{n} .
$$

Therefore $x=a \cup c$ (or better: $\operatorname{ran}(x)=\operatorname{ran}(a) \cup \operatorname{ran}(c)), y=b \cup c$, hence $x \cup y=$ $a \cup b \cup c, x \cap y=c, D(2 ; x)=a \backslash c=a$ and $D(2 ; y)=b \backslash c=b$, and therefore $\bigcup_{n}\left[F(x)_{n}\right] \neq \bigcup_{n}\left[F(y)_{n}\right]$. We are out to show a contradiction from the assumption that $\bigcup_{n}\left[F(x)_{n}\right] \triangle \bigcup_{n}\left[F(y)_{n}\right] \neq \emptyset$; for the sake of definitiveness suppose there is some

$$
w \in \bigcup_{n}\left[F(x)_{n}\right] \backslash \bigcup_{n}\left[F(y)_{n}\right] .
$$

Let $p$ be a function $p: A \rightarrow \mathbb{R}$ with $A \subset \omega \times 2$ finite. Then

$$
\begin{aligned}
C(p) & =\left\{x \in{ }^{\omega \times 2} \mathbb{R} \mid \forall(n, i) \in A\left(x_{n, i}=p_{n, i}\right)\right\} \\
& =\left\{x \in{ }^{\omega \times 2} \mathbb{R} \mid x \supset p\right\}
\end{aligned}
$$

is a closed subset of ${ }^{\omega \times 2} \mathbb{R}$. We say that $p$ is $x$-restricted iff

$$
\begin{aligned}
& (n, 0) \in A \Longrightarrow p_{n, 0} \in\left\{x_{m, i} \mid(m, i) \in \omega \times 2\right\} \\
& (n, 1) \in A \Longrightarrow p_{n, 1} \in\left\{c_{m} \mid m \in \omega\right\}
\end{aligned}
$$

Thus an $x$-restricted $p$ is an approximation to a $z$ with $D(2 ; z)=D(2 ; x)$ - see Lemma 37 for the precise statement.

Claim 36. If $p$ is $x$-restricted, $U \subseteq{ }^{\omega \times 2} \mathbb{R}$ is open, and $U \cap C(p) \neq \emptyset$, then there is $q \supset p$ such that $q$ is $x$-restricted and $C(q) \subseteq U \cap C(p)$.

Proof. Without loss of generality we may assume that $U$ is a basic open set of the form

$$
U=\prod_{(n, i) \in B} V_{n, i} \times \prod_{(n, i) \in \omega \times 2 \backslash B} \mathbb{R}
$$

where $B \subset \omega \times 2$ is finite and the $V_{n, i} \subseteq \mathbb{R}$ are open and non-empty. By case assumption $p_{n, i} \in V_{n, i}$ for $(n, i) \in B \cap \operatorname{dom}(p)$, so we can define $q: B \cup \operatorname{dom}(p) \rightarrow \mathbb{R}$ to be $p$ on $\operatorname{dom}(p)$ and for $(n, i) \in B \backslash \operatorname{dom}(p)$ set $q(n, i)=c_{i}$, where $i$ is least such that $c_{i} \in V_{n, i}$. Such an $i$ exists by density of $c$.

For a fixed $x$-restricted $p$ consider the following game $\mathcal{G}(p)$ : I plays $x$-restricted $p_{n}$ 's and II plays open sets $U_{n} \subseteq{ }^{\omega \times 2} \mathbb{R}$ subject to the rules that

- $\operatorname{diam}\left(U_{n}\right)<2^{-n}$ and $U_{n} \cap C\left(p_{n}\right) \neq \emptyset$,

- $p=p_{0}, p_{n} \subseteq p_{n+1}$, and $C\left(p_{n+1}\right) \subseteq U_{n} \cap C\left(p_{n}\right)$.

(The diameters of the $U_{n}$ 's is taken with respect to a complete metric on ${ }^{\omega \times 2} \mathbb{R}$ compatible with the product topology.) The first player to violate these rules loses. If $\left\langle\left(p_{n}, U_{n}\right) \mid n \in \omega\right\rangle$ is a complete play of $\mathcal{G}(p)$ then $\bigcap_{n} C\left(p_{n}\right)$ is a singleton $\{z\}$, since the $C\left(p_{n}\right)$ 's are closed and of shrinking diameter. Then I wins $\mathcal{G}(p)$ iff $D(2 ; z)=$ $D(2 ; x)$.

Lemma 37. If $p$ is $x$-restricted, then $\boldsymbol{I}$ has a winning strategy for $\mathcal{G}(x, p)$. 
Proof. Let $\Sigma$ be the following strategy for $\mathbf{I}$ : Set $\Sigma(\emptyset)=p_{0}=p$, and suppose $\left\langle p_{0}, U_{0}, \ldots, p_{n}\right\rangle$ is a position according to $\Sigma$ and that neither player has violated the rules so far. Suppose $U_{n}$ is open, of diameter $<2^{-n}$ and $U_{n} \cap C\left(p_{n}\right) \neq \emptyset$. By Claim 36 there is an $x$-restricted $q \supseteq p$ such that $C(q) \subseteq U_{n} \cap C\left(p_{n}\right)$. We now define $\Sigma$ 's response to $U_{n}$ to be the function $p_{n+1}$ obtained by extending $q$ as follows: choose $(h, 0),(k, 1) \notin \operatorname{dom}(q)$ and set $p_{n+1}(h, 0)=a_{n}$ and $p_{n+1}(k, 1)=c_{n}$. Therefore $\left\langle\left(p_{n}, U_{n}\right) \mid n \in \omega\right\rangle$ is a play according to $\Sigma$ in which II has followed the rules, and if $z$ is the unique element of $\bigcap_{n} C\left(p_{n}\right)$, then $D(2 ; z)=\left\{a_{n} \mid n \in \omega\right\}$, which is what we had to prove.

Lemma 38. There is an $x$-restricted $p$ and $a k<\omega$, such that

$$
\forall z \in C(p) w \in\left[F(z)_{k}\right] .
$$

Proof. Suppose not. We will construct a $\bar{z} \in{ }^{\omega \times 2} \mathbb{R}$ such that

(a) $D(2 ; \bar{z})=D(2 ; x)$ and

(a) $w \notin \bigcup_{n}\left[F(\bar{z})_{n}\right]$.

This is a contradiction, since $w \in \bigcup_{n}\left[F(x)_{n}\right]$ and $\bigcup_{n}\left[F(x)_{n}\right]=\bigcup_{n}\left[F(\bar{z})_{n}\right]$ by (a) above and by assumption on $F$. We will construct a complete play $\left\langle\left(p_{n}, U_{n}\right) \mid n \in \omega\right\rangle$ according to a winning strategy for $\mathbf{I}$ in $\mathcal{G}(\emptyset)$ and will take $\bar{z}$ to be the unique element of $\bigcap_{n} C\left(p_{n}\right)$ so that (a) will follow from Lemma 37. (Note that the empty function is $x$-restricted.) We will maintain that $\forall z \in C\left(p_{n}\right) w \notin\left[F(z)_{n}\right]$ : this will imply that $w \notin \bigcup_{k}\left[F(\bar{z})_{k}\right]$, establishing thus (b). Therefore it is enough to construct the $U_{n}$ 's so that II never violates the rules of $\mathcal{G}(\emptyset)$. Suppose $\left\langle p_{0}, U_{0}, \ldots, p_{n}\right\rangle$ is a position of $\mathcal{G}(\emptyset)$ according to $\Sigma$ and that II has not broken the rules so far. Since $F$ is continuous, the set $V_{n}=\left\{z \in{ }^{\omega \times 2} \mathbb{R} \mid w \notin\left[F(z)_{n}\right]\right\}$ is open. By case assumption $C\left(p_{n}\right) \cap V_{n} \neq \emptyset$, so II can simply shrink $V_{n}$ to a $U_{n}$ with $\operatorname{diam}\left(U_{n}\right)<2^{-n}$ and $C\left(p_{n}\right) \cap U_{n} \neq \emptyset$.

We can now reach a contradiction, establishing thus Lemma 35. Fix $p$ and $k$ as in Lemma 38 and choose $z \supset p$ such that $\left\{z_{n, 0} \mid(n, 0) \notin \operatorname{dom}(p)\right\}=\left\{b_{n} \mid n \in \omega\right\}$ and $\left\{z_{n, 1} \mid(n, 1) \notin \operatorname{dom}(p)\right\}=\left\{p_{n, 0} \mid(n, 0) \in \operatorname{dom}(p)\right\}$. Then $D(2 ; z)=b=D(2 ; y)$ and hence $\bigcup_{n}\left[F(z)_{n}\right]=\bigcup_{n}\left[F(y)_{n}\right]$. But $z \in C(p)$, so $w \in\left[F(z)_{k}\right] \subseteq \bigcup_{n}\left[F(y)_{n}\right]$, contradicting our choice of $w$.

Suppose now $F:{ }^{\omega \times 2} \mathrm{P} \operatorname{Tr} \rightarrow{ }^{\omega} \mathrm{PTr}$ is a function (not necessarily continuous) that induces an injection of $2-\Sigma_{2}^{0}$ into $\Sigma_{2}^{0}$. By determinacy $F$ is continuous on a comeager $\mathcal{G} \subseteq{ }^{\omega \times 2} \mathrm{PTr}$. Unfortunately we cannot apply the technique of Lemma 35, since $\mathcal{G}$ need not to contain codes for (differences of) countable sets, nor to be closed under equivalences of codes, that is, $\pi_{2}(x)=\pi_{2}(y)$ and $x \in \mathcal{G}$ does not imply $y \in \mathcal{G}$. The solution is to continuously re-interpret the codes for $2-\Sigma_{2}^{0}$ inside $\mathcal{G}$, that is construct a continuous $G:{ }^{\omega \times 2} \mathrm{PTr} \rightarrow \mathcal{G}$ such that

$$
\pi_{2}(x)=\pi_{2}(y) \Longleftrightarrow \pi_{2}(G(x))=\pi_{2}(G(y))
$$

and then compose with $F$ (which is continuous on $\mathcal{G}$ ) and appeal to Lemma 35 to reach a contradiction.

Theorem 39 (ZF $+\mathrm{DC})$. Let $\mathcal{G} \subseteq{ }^{\omega \times 2} \mathrm{P} \operatorname{Tr}$ be comeager. Then there exist 
- a continuous $G:{ }^{\omega \times 2} \mathrm{P} \operatorname{Tr} \rightarrow \mathcal{G}$,

- a pruned tree $W$,

- a Lipschitz homeomorphism $H: \mathbb{R} \rightarrow[W]$, and

- an $\mathbf{F}_{\sigma}$ set $J \subseteq \mathbb{R} \backslash[W]$,

such that for each $\left\langle T_{n, i} \mid(n, i) \in \omega \times 2\right\rangle \in{ }^{\omega \times 2} \mathrm{PTr}$, letting $\left\langle T_{n, i}^{\prime} \mid(n, i) \in \omega \times 2\right\rangle=$ $G\left(\left\langle T_{n, i} \mid(n, i) \in \omega \times 2\right\rangle\right) \in \mathcal{G}$, the part of $\bigcup_{n}\left[T_{n, 0}^{\prime}\right] \backslash \bigcup_{n}\left[T_{n, 1}^{\prime}\right]$ in $[W]$ is homeomorphic to $\bigcup_{n}\left[T_{n, 0}\right] \backslash \bigcup_{n}\left[T_{n, 1}\right]$ via $H$, and the part outside $[W]$ is equal to $J$, that is

$$
\begin{aligned}
& \left(\bigcup_{n}\left[T_{n, 0}^{\prime}\right] \backslash \bigcup_{n}\left[T_{n, 1}^{\prime}\right]\right) \cap[W]=H \text { " }\left(\bigcup_{n}\left[T_{n, 0}\right] \backslash \bigcup_{n}\left[T_{n, 1}\right]\right), \\
& \left(\bigcup_{n}\left[T_{n, 0}^{\prime}\right] \backslash \bigcup_{n}\left[T_{n, 1}^{\prime}\right]\right) \backslash[W]=J .
\end{aligned}
$$

It is not hard to see that (63) implies (62). Since AD implies that every set in the plane can be uniformized on a comeager set, then it can be shown that any injection $2-\Sigma_{2}^{0} \longmapsto \Sigma_{2}^{0}$ is induced by a continuous $F: \mathcal{G} \rightarrow{ }^{\omega} \mathrm{PTr}$ on a comeager $\mathcal{G}$, and therefore Theorem 39 will imply that

$$
\mathrm{AD} \Longrightarrow\left|\Sigma_{2}^{0}\right|<\left|2-\Sigma_{2}^{0}\right|
$$

which is what we wanted to show.

The proof of Theorem 39 is rather technical and will not be given here, but it is based on a Baire-category argument, just like the proof of Theorem 29.

\section{REFERENCES}

[And03] Alessandro Andretta. Equivalence between Wadge and Lipschitz determinacy. Annals of Pure and Applied Logic, 123:163-192, 2003.

[Hjo98] Greg Hjorth. An absoluteness principle for Borel sets. The Journal of Symbolic Logic, 63:663-693, 1998.

[Hjo02] Greg Hjorth. Cardinalities in the projective hierarchy. The Journal of Symbolic Logic, 67:1351-1372, 2002.

[Kec95] Alexander S. Kechris. Classical Descriptive Set Theory. Number 156 in Graduate Texts in Mathematics. Springer-Verlag, Heidelberg, New York, 1995.

[VW77] Robert A. Van Wesep. Subsystems of Second-Order Arithmetic and Descriptive Set Theory under the Axiom of Determinateness. PhD thesis, University of California, Berkeley, 1977.

[VW78] Robert A. Van Wesep. Wadge degrees and descriptive set theory. In Alexander S. Kechris and Yiannis N. Moschovakis, editors, Cabal Seminar 76-77, number 689 in Lecture Notes in Mathematics. Springer-Verlag, 1978.

[Wad83] William W. Wadge. Reducibility and Determinateness on the Baire Space. PhD thesis, University of California, Berkeley, 1983.

Dipartimento di Matematica, Universitì di Torino, Italy

E-mail address: andretta@dm.unito.it

Department of Mathematics, University of California at Los Angeles

E-mail address: greg@math.ucla.edu

Department of Mathematics, University of California at Los Angeles

E-mail address: ineeman@math.ucla.edu 